\title{
Negligible surface uplift following foundering of thickened Central Tibetan lower crust
}

\author{
Yunchuan Zeng, Mihai N. Ducea, Jifeng Xu, Jianlin Chen, Yanhui Dong \\ Corresponding to Jifeng Xu (jifengxu@gig.ac.cn; jifengxu@cugb.edu.cn)
}

\section{FIGURES}

Figure S1- (a) simplified geological map showing the sample location; (b) Filed photo showing EARs overlying on early Tertiary fluviolacustrine strata

Figure S2-Microphotograph for the EARs in this study.

Figure $\mathrm{S} 3$-Zircon concordant $\mathrm{U}-\mathrm{Pb}$ age diagram and representative cathodoluminescence images for the EARs.

Figure S4- Selective elemental hacker diagrams for the EARs.

Figure S5- Sample-standard bracketing of measured Hf and O isotopic results during analyses.

\section{APPENDICES}

Experimental Methods

\section{TABLES}

Table S1. Zircon U-Pb isotope data for the EARs

Table $\mathrm{S} 2$. $\mathrm{Lu}-\mathrm{Hf}$ and $\mathrm{O}$ isotope data for zircon grains from the EARs

Table S3. Bulk-rock major and trace element data for the EARs in this study

Table S4. Bulk-rock Sr-Nd isotope data for EARs in this study

Table S5. In-situ major elements for cpx measured by EPMA

Table S6. In-situ major and trace elements for cpx measured by LA-ICP-MS

Table S7-Published data for EARs

Table S8-Metadata of LA-MC-ICPMS for U-Pb and Lu-Hf isotope analyses used in this study 


\section{FIGURES}

Figure S1-(a) simplified geological map showing the sample location; (b) Filed photo showing EARs overlying on early Tertiary fluviolacustrine strata (Suonahu Formation) that occurs as flat beds and experienced little or no N-S shortening in the Zhuozishan region at modern elevation of $\sim 5200 \mathrm{~m}$. The depositional age of Suonahu Formation is a long time of debate, but has been recently well constrained by SIMS (secondary ion mass spectroscopy) zircon U-P b dating (46.57 $\pm 0.30 \mathrm{Ma}$ ) of bentonite layers drilled at 220.8 220.95m depth in the Bandonghu region (Wang et al., 2019). This demonstrates that shortening ceased, and that low-relief topographic conditions were achieved in this region by $\sim 46 \mathrm{Ma}$.
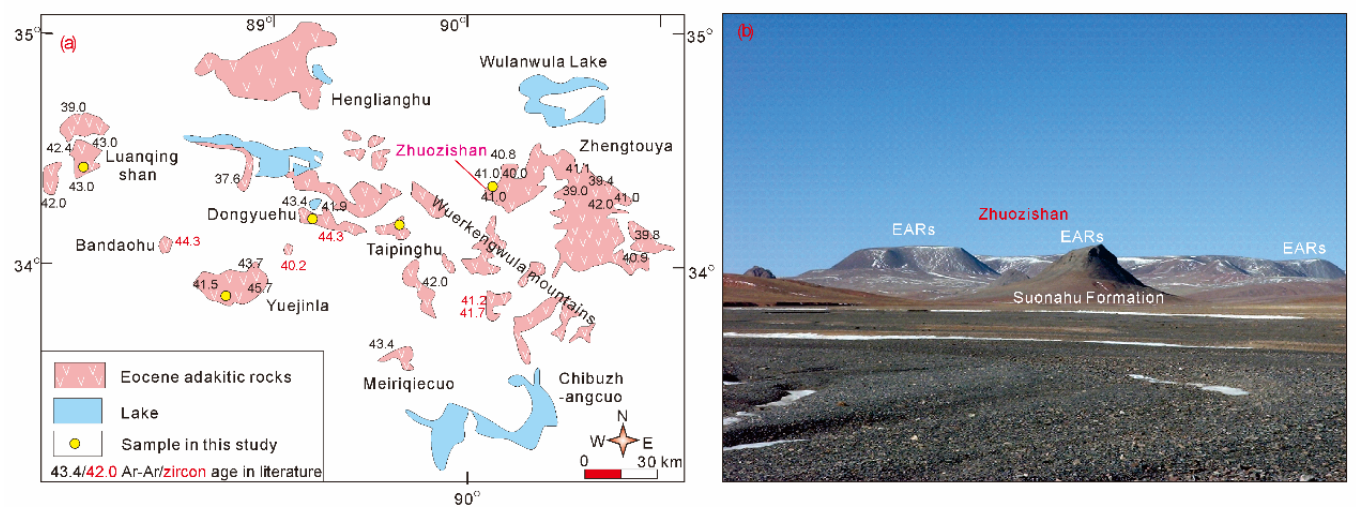

\section{Reference}

Wang, J., Zeng, S.Q., Fu, X.G., Chen, W.B., Dai, J., Ren, J., 2019, New evidence for deposition age of the Suonahu Formation in the Qiangtang Basin. Geological Bulletin of China, 2019, $38(7), 1256-1258$. 
Figure S2-Microphotograph for the EARs in this study (Fig. i is under backscattered electron images). $\mathrm{Cpx}=$ clinopyroxene, $\mathrm{Pl}=$ plagioclase, $\mathrm{Bi}=$ biotite, $\mathrm{Q}=$ quartz, $\mathrm{Am}=$ amphibole, $\mathrm{Sa}=$ sanidine. The red line in Fig. i shows the compositional profile measured by EPMA. The results are listed in Table S5, which show similar variational trend with YJL-24 (not shown).
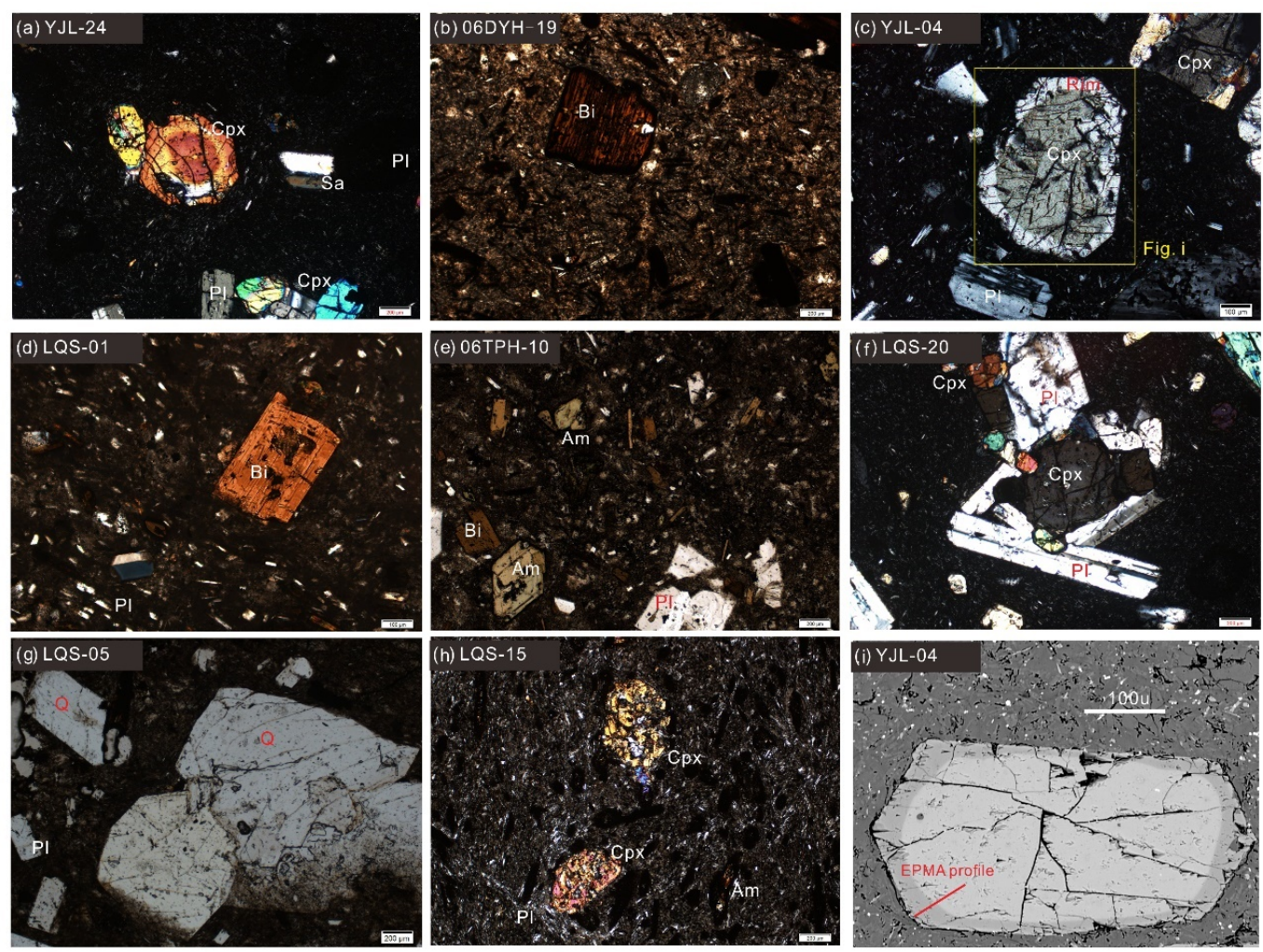
Figure S3-Zircon concordant $\mathrm{U}-\mathrm{Pb}$ age and representative cathodoluminescence images for the EARs. The yellow, red and blue circles in Fig. e show the positions for $\mathrm{U}-\mathrm{Pb}, \mathrm{Lu}-\mathrm{Hf}$ and $\mathrm{O}$ isotope analysis, respectively.
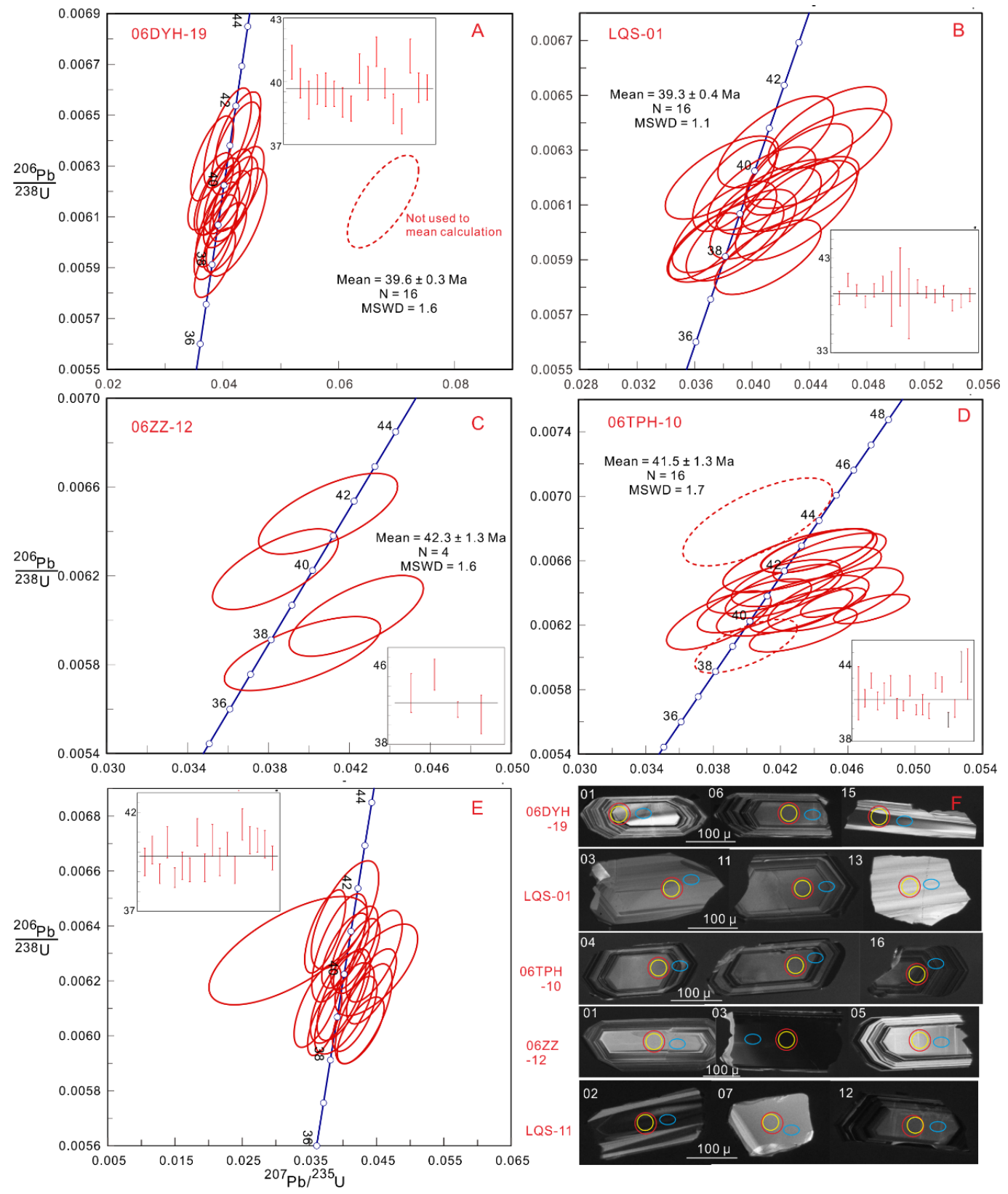
Figure S4- Selective elemental hacker diagram for the EARs. The data for the published EARs are given in the Table S7. The data for the oceanic adakites are from Castillo (2012). Although not shown here, the trace elements, like $\mathrm{Y}, \mathrm{Zr}$, Th and $\mathrm{Ba}$ of the EARs are also in good correlation with $\mathrm{SiO}_{2}$. The $\mathrm{A} / \mathrm{CNK}$ in Fig. $\mathrm{f}$ refers to peraluminous index $(\mathrm{A} / \mathrm{CNK}=$ molar proportions $\left.\mathrm{Al}_{2} \mathrm{O}_{3} /\left(\mathrm{Na}_{2} \mathrm{O}+\mathrm{K}_{2} \mathrm{O}+\mathrm{CaO}\right)\right)$. The positive correlation between $\mathrm{A} / \mathrm{CNK}$ and $\mathrm{SiO} 2$ indicate sediment assimilation with possible impact of fractional crystallization (e.g., removal of amphibole; Clemens et al., 2011).
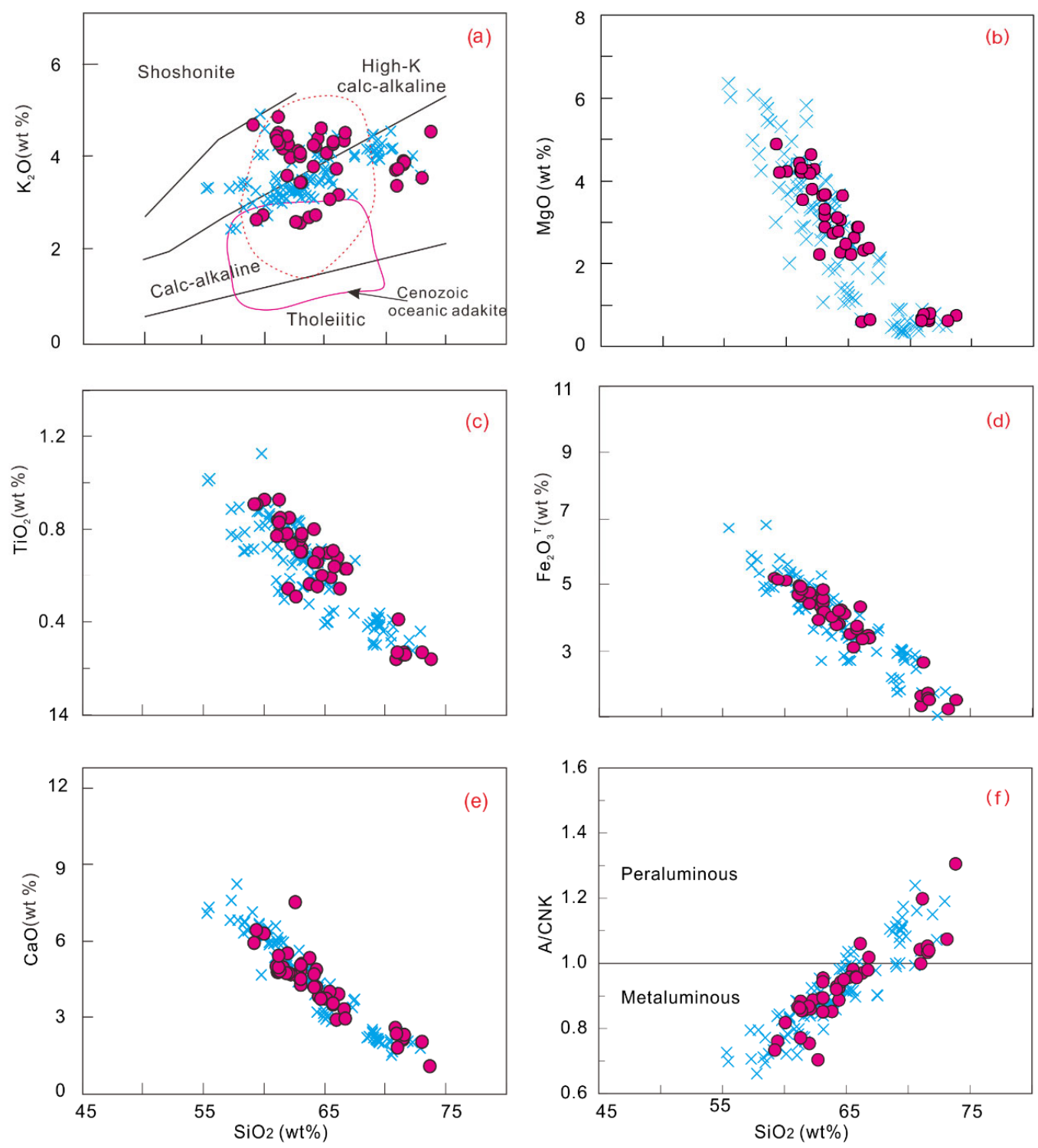

References:

Castillo, P.R., Adakite petrogenesis, 2012: Lithos, v. 134-135, p. 304-316, https://doi.10.1016/j.lithos.2011.09.013.

Clemens, J.D., Stevens, G., Farina, F., The enigmatic sources of I-type granites: The peritectic connexion, 2011: Lithos, v. 126, p. 174-181, https://doi:10.1016/j.lithos.2011.07.004. 
Figure S5-Sample-standard bracketing of measured Hf and O isotopic results during analyses.
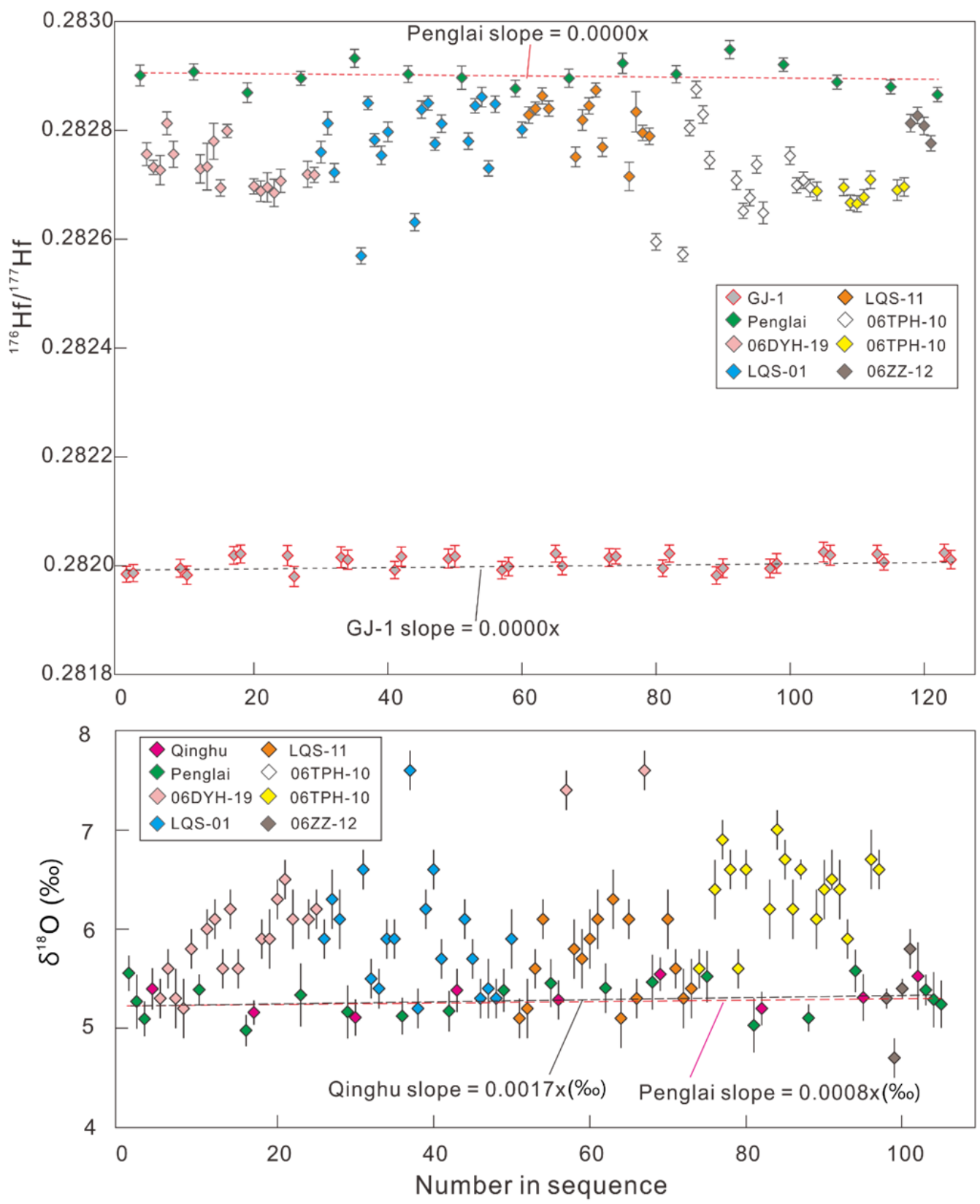


\section{APPENDIX. EXPERIMENTAL METHODS \\ In-Situ Zircon U-Pb, Lu-Hf And O Isotope Analysis}

Zircon grains were separated by standard density and magnetic separation techniques, and individually selected by hand-picking under a binocular microscope. Zircon grains were then inlaid in epoxy blocks, and polished to smooth surface. Cathodoluminescence (CL) images are used to select the appropriate grains for analysis. Zircon $\mathrm{U}-\mathrm{Pb}$ isotope analyses were performed by laser ablation inductively coupled plasma mass spectrometry (LA-ICP-MS) at the Guangxi Key Laboratory of Hidden Metallic Ore Deposits Exploration, Guilin University of Technology (GUT), Guilin, China. Laser sampling was performed using a Coherent GeoLas 193 ArF excimer. An Agilent 7500a ICP-MS instrument was used to acquire ion-signal intensities. Detailed metadata related to the laboratory and sample preparation methods, following the guidelines in http://www.plasmage.org/recommendations and recommended in Horstwood et al. (2016) and thte metadata of LA-MC-ICPMS U-Pb and Lu-Hf data used are listed in Table S8. Helium was used as a carrier gas. Argon was used as the make-up gas and mixed with the carrier gas via a T-connector before entering the ICP. Following about $20 \mathrm{~s}$ period of background analysis, samples were spot ablated for $60 \mathrm{~s}$ at a $6 \mathrm{~Hz}$ repetition rate with a spot size of $32 \mu \mathrm{m}$ beam and laser energy of 10 $\mathrm{J} / \mathrm{cm}^{-2}$ at the sample surface. Plesovice zircon standard ${ }^{206} \mathrm{~Pb}{ }^{238} \mathrm{U}$ age $=337 \mathrm{Ma}$; Slám et al., 2008 ) and NIST 610 were used as isotope and trace elements external standards, respectively. GJ1 standard zircon $\left({ }^{206} \mathrm{~Pb} /{ }^{238} \mathrm{U}\right.$ age $=608.5 \pm 0.4$; Jackson et al., 2004) was used as isotope internal standards and unknow samples. Off-line inspection and integration of background and analyte signals, and time-drift correction and quantitative calibration for trace element analyses and U-Pb dating were performed using ICPMSDataCal software (Liu et al., 2008; Liu et al., 2010), and the results were reported with $2 \sigma$ errors. Reported uncertainties $(2 \sigma)$ of the ${ }^{206} \mathrm{~Pb} /{ }^{238} \mathrm{U}$ ratio were propagated by quadratic addition of the external reproducibility (2 SD\%) of standard zircon Plesovice during the analytical session and the within-run precision of each analysis (2 SE\%; standard error) (Liu et al. 2008; Liu et al., 2010). The common $\mathrm{Pb}$ was corrected using the method proposed by Andersen (2002). Final assessment of uncertainty, concordia diagrams and weighted mean calculation were done by using Isoplot ExcelTM (Ludwig, 2012) and following the recommendation of Horstwood et al. (2016) and Spencer et al. (2016). Errors are quoted at the $2 \sigma$ level. During the course of this study, the analyzed GJ-1 standard zircon grains yielded a weighted ${ }^{206} \mathrm{~Pb} /{ }^{238} \mathrm{U}$ age of $605 \pm 3 \mathrm{Ma}(2 \sigma, \mathrm{MSWD}=0.4, \mathrm{n}=24$; Table $\mathrm{S} 1)$, which is in agreement with the recommended U-Pb age in allowed precision. One spot of 06DYH-19 is discordant, and two spots of sample $06 \mathrm{TPH}-10$ have ${ }^{206} \mathrm{~Pb} /{ }^{238} \mathrm{U}$ age that are overdispersion as defined by Spencer et al. (2016), which are therefore are not used to calculate the weighted averages.

Zircon Lu-Hf isotopes are performed by using a Neptune Plus MC-ICP-MS equipped with a laser ablation system at GST. The $\mathrm{Lu}-\mathrm{Hf}$ isotopic measurements were made on the spots which were previously analyzed for $\mathrm{U}-\mathrm{Pb}$ ages as much as possible. The analyses were conducted with a beam diameter of $44 \mu \mathrm{m}$. Normally, a signal intensity of $>5 \mathrm{~V}$ at ${ }^{180} \mathrm{Hf}$ mass can be obtained using the laser repetition rate of $6 \mathrm{~Hz}$ with energy of $10 \mathrm{Jcm}^{-2}$. The ablated material was transported in a helium carrier gas with addition of a small flow of nitrogen. Data acquisition for each analysis consists of $20 \mathrm{~s}$ period of background analysis and about $47 \mathrm{~s}$ period of laser ablation. The measured isotopic ratios of ${ }^{176} \mathrm{Hf} /{ }^{177} \mathrm{Hf}$ were normalized to ${ }^{179} \mathrm{Hf} /{ }^{177} \mathrm{Hf}=0.7325$, using exponential correction for mass bias. In order to obtain accurate ${ }^{176} \mathrm{Hf} /{ }^{177} \mathrm{Hf}$ ratios, the isobaric interferences of ${ }^{176} \mathrm{Lu}$ and ${ }^{176} \mathrm{Yb}$ on ${ }^{176} \mathrm{Hf}$ must be corrected. The ratios of ${ }^{176} \mathrm{Lu} /{ }^{175} \mathrm{Lu}=$ $0.02655,{ }^{172} \mathrm{Yb} /{ }^{176} \mathrm{Yb}=0.5886, \beta \mathrm{Yb}=0.8725 \times \beta \mathrm{Hf}$ obtained during $\mathrm{Hf}$ analysis on the same spot were used in the isobaric interference correction. Two GJ-1 and one Penglai zircon standard were analyzed after every five specimens as isotope external standards and unknown samples, respectively. The ${ }^{176} \mathrm{Hf} /{ }^{177} \mathrm{Hf}$ value for GJ-1 zircon grains during the analytical session ranges from $0.281984 \pm 19$ to $0.282025 \pm 18$ with a weighted average value of $0.282005 \pm$ $0.000005(2 \sigma ; \mathrm{MSWD}=0.75 ; \mathrm{n}=32)$, consistent with the reference value of $0.282015 \pm 0.000019$ $(2 \sigma)$ within the analytical error (Elhlou et al., 2006). The ${ }^{176} \mathrm{Hf} /{ }^{177} \mathrm{Hf}$ value of analyzed Penglai zircon grains ranges from $0.282869 \pm 16$ to $0.282921 \pm 17$ with a weighted average value of $0.282897 \pm 9(2 \sigma, \mathrm{n}=16, \mathrm{MSWD}=0.52)$, which is consist with the reference value within analytical errors $\left(0.282906 \pm 0.000010\right.$; Li et al., 2010). The stable ${ }^{178} \mathrm{Hf} /{ }^{177} \mathrm{Hf}$ and ${ }^{180} \mathrm{Hf} /{ }^{177} \mathrm{Hf}$ ratios of GJ-1 grains yielded values of $1.46716 \pm 0.00008$ and $1.88683 \pm 0.00009(2 \sigma, \mathrm{n}=32)$, the stable ${ }^{178} \mathrm{Hf} /{ }^{177} \mathrm{Hf}$ and ${ }^{180} \mathrm{Hf} /{ }^{177} \mathrm{Hf}$ ratios of PengLai grains yielded values of $1.46745 \pm 11$ and 
$1.88618 \pm 11(2 \sigma, \mathrm{n}=16)$, respectively, and are within $200 \mathrm{ppm}$ of known values based upon atomic masses and abundances as recommended by Spencer et al. (2020). Thus, these data demonstrate the instrument was working properly.

Zircon oxygen isotopes were measured using Cameca IMS 1280 large-radius SIMS (Secondary Ion Mass Spectroscopy) at Guangzhou Institute of Geochemistry, Chinese Academy of Sciences (GIGCAS). The detailed analytical procedures were similar with those described by Li et al. (2010). The normal-incidence electron flood gun was used to compensate for sample charging during analysis with homogeneous electron density over a $100 \mu \mathrm{m}$ oval area. Negative secondary ions were extracted with a $-10 \mathrm{kV}$ potential. The field aperture was set to $5000 \mu \mathrm{m}$, and the transfer-optics magnification was adjusted to give a field of view of $125 \mu \mathrm{m}(\mathrm{FA}=8000)$. Energy slit width was $30 \mathrm{eV}$, the mechanical position of the energy slit was controlled before starting the analysis ( $5 \mathrm{eV}$ gap, -500 digits with respect to the maximum). The entrance slit width is $\sim 120 \mu \mathrm{m}$ and exit slit width for multicollector Farady cups (FCs) for $16 \mathrm{O}$ and ${ }^{18} \mathrm{O}$ is $500 \mu \mathrm{m}$ $(\mathrm{MRP}=2500)$. The intensity of ${ }^{16} \mathrm{O}$ was typically $1 \times 109 \mathrm{cps}$. Oxygen isotopes were measured in multi-collector mode using two off-axis Faraday cups. The NMR (Nuclear Magnetic Resonance) probe was used for magnetic field control with stability better than $2.5 \mathrm{ppm}$ over $16 \mathrm{~h}$ on mass 17 . One analysis takes $\sim 4$ minutes, consisting of pre-sputtering $(\sim 120 \mathrm{~s})$, automatic beam centering $(\sim 60 \mathrm{~s})$ and integration of oxygen isotopes $(10$ cycles $\times 4 \mathrm{~s}$, total $40 \mathrm{~s})$. Uncertainties on individual analyses are reported at a $2 \sigma$ level. With low noise on the two FC amplifiers, the internal precision of a single analysis is generally better than $0.3 \%(2 \sigma)$ for ${ }^{18} \mathrm{O} /{ }^{16} \mathrm{O}$ ratio. Values of $\delta^{18} \mathrm{O}$ are standardized to Vienna Standard Mean Ocean Water compositions (VSMOW) and reported in standard per mil notation. The measured oxygen isotopic data were corrected for instrumental mass fractionation using the Penglai zircon standard $\left(\delta^{18} \mathrm{O}_{\text {VSMOW }}=5.31 \pm 0.10 \%\right.$ o $)$ (Li et al., 2010). The PengLai zircon standard during the course of this study yield a weighted mean of $\delta^{18} \mathrm{O}$ $=5.27 \pm 0.09 \%(2 \sigma, \mathrm{MSWD}=0.84, \mathrm{n}=20$; Table S1), which is identical within errors to the recommended value ( $\mathrm{Li}$ et al., 2010). Measured ${ }^{18} \mathrm{O} /{ }^{16} \mathrm{O}$ is normalized using VSMOW, then corrected for the instrumental mass fractionation factor (IMF) as follows: $\left(\delta^{18} \mathrm{O}\right) \mathrm{M}=$

$\left(\left({ }^{18} \mathrm{O} /{ }^{16} \mathrm{O}\right)_{\mathrm{M}} / 0.0020052-1\right) \times 1000(\%) ; \mathrm{IMF}=\left(\delta^{18} \mathrm{O}\right)_{\mathrm{M}(\text { standard })}-\left(\delta^{18} \mathrm{O}\right)_{\mathrm{VSMOW}} ; \delta^{18} \mathrm{O}_{\text {sample }}=$ $\left(\delta^{18} \mathrm{O}\right)_{\mathrm{M}}+\mathrm{IMF}$. Besides, Qinghu zircon grains analyzed as unknown samples yielded a weighted mean of $\delta^{18} \mathrm{O}=5.28 \pm 0.12 \%(2 \sigma, \mathrm{MSWD}=0.70, \mathrm{n}=9$; Table $\mathrm{S} 3)$, which is consistent with the recommended value of $5.4 \pm 0.2 \%$ within analytical errors ( $\mathrm{Li}$ et al., 2013). Results of Hf and O isotope data of standard and samples are graphically plotted in Fig. S5.

\section{Bulk-Rock Element and Sr-Nd Isotope Analysis}

Bulk-rock geochemical data were performed at GIGCAS. Firstly, fresh sample were crushed into small grains $(\sim 0.5 \mathrm{~cm})$, and soaked in water with $<3 \% \mathrm{HNO}_{3}$ until no bubbles were observed. Then, these small grains were further crushed into 200 meshes in an agate mortar. About $1.2 \mathrm{~g}$ rock powder were baked in the muffle furnace for more than 3 hours at $915{ }^{\circ} \mathrm{C}$ to determine the loss-on-ignition (LOI) value. Then about $0.5 \mathrm{~g}$ baked samples were fluxed with $\mathrm{Li}_{2} \mathrm{~B}_{4} \mathrm{O}_{7}(1: 8)$ at $\sim 1200^{\circ} \mathrm{C}$ to make homogeneous glass disks for major elements determination with a Rigaku ZSX100e X-ray fluorescence spectrometer (XRF). During analysis, USGS rock reference materials (GSR-1, GSR-2 and GSR-3) were used to monitor the data quality. The analytical uncertainties are estimated at $1-5 \%$.

For trace elements analysis, 35-45 mg sample powders were dissolved in Teflon bakers using a hybrid acid for $2-3$ days $\left(\mathrm{HF}, \mathrm{HNO}_{3}\right.$ and $\mathrm{HClO}_{4}$ proportions are $\left.2: 2: 1\right)$ at $120{ }^{\circ} \mathrm{C}$. Then the solutions were dried and the residues were dissolved using the same hybrid acid again and moved in the high-pressure bombs at $190{ }^{\circ} \mathrm{C}$ for 48 hours. After that, the sample solutions were dried again and dissolved with $50 \% \mathrm{HNO}_{3}$ in the bombs at $170{ }^{\circ} \mathrm{C}$ for 4 hours. Subsequently, the sample solutions were diluted by $3 \% \mathrm{HNO}_{3}$ to 2000 times. Then, about $2.5 \mathrm{~g}$ diluted solution was mixed with the internal standard solution (pure single element Rh solution) at 1:1 ratio. Finally, the hybrid solutions were used to trace-element data measures by inductively coupled plasmamass spectrometry (ICP-MS). During the procedure, USGS international materials (BHVO-2, AGV-1, GSR-1, GSR-2, GSR-3, W-2, SY-4) and two blank samples were taken as the external calibration standards. The analytical precision was generally better than $10 \%$.

Powdered samples $(\sim 100 \mathrm{mg})$ for $\mathrm{Sr}$ and $\mathrm{Nd}$ isotopic analyses were dissolved using an acidic mixture of $\mathrm{HF}$ and $\mathrm{HNO}_{3}$ (1:3) for 7 days at $120{ }^{\circ} \mathrm{C}$ in PFA beakers. The Sr-REE and Nd-REE separation were performed using cationic ion-exchange columns and HDEHP columns, 
respectively. The powders for $\mathrm{Hf}$ isotopic analysis were dissolved by alkali fusion method which a mixture of about $0.5 \mathrm{~g}$ rock powders and $1.0 \mathrm{~g} \mathrm{Li}_{2} \mathrm{~B}_{4} \mathrm{O}_{7}$ was made into glass disk similar with that of the major element analysis. Then the powders of alkali glass were dissolved in $2 \mathrm{M} \mathrm{HCl}$ at $\sim 60{ }^{\circ} \mathrm{C}$ for about 2 hours. Hf fraction was separated by using a modified single-column Ln extraction chromatography method. The $\mathrm{Sr}-\mathrm{Nd}$ isotopic data were obtained on multi-collector inductively-coupled plasma mass spectrometer (MC-ICP-MS) at GIGCAS, and normalized values are ${ }^{86} \mathrm{Sr} /{ }^{88} \mathrm{Sr}=0.1194$ and ${ }^{146} \mathrm{Nd} /{ }^{144} \mathrm{Nd}=0.7219$, respectively, while the ${ }^{87} \mathrm{Rb} /{ }^{86} \mathrm{Sr}$ and ${ }^{147} \mathrm{Sm} /{ }^{144} \mathrm{Nd}$ ratios were calculated from abundances of these trace elements measured by ICP-MS. The analytical procedure method is the same as in Chen et al. (2010).

\section{In-situ cpx composition Analysis}

Major elements were measured on individual minerals using a JEOL JXA-8100 electron microscope (EPMA) with an accelerating potential of $15 \mathrm{kV}$ and sample current of $10 \mathrm{nA}$ at the Institute of Geology and Geophysics, Chinese Academy of Sciences (IGGCAS). The detailed method has been described in Liu et al. (2010).

The major and trace elements of cpx were also measured using laser ablation inductively coupled plasma mass spectrometry (LA-ICP-MS) with a spot size of $35 \mu \mathrm{m}$ at the State Key Laboratory of Geological Process and Mineral Resource, China University of Geology (Wuhan). Detailed operating conditions for the laser ablation system and the ICP-MS instrument were described previously (Liu et al., 2008) and are the same with later studies like Zou et al. (2014). Off-line selection and integration of the background and the analyzed signals, along with timedrift correction and quantitative calibration, were performed by ICPMSDataCal (Liu et al., 2008, 2010).

Notably, within the analytical errors, the major elements obtained by EPMA and LA-ICP-MS in the same position are comparable, therefore both of them are used to discussion directly.

\section{References}

Andersen, T. (2002) Correction of common lead in U-Pb analyses that do not report 204Pb. Chemical Geology, 192, 59-79.

Black, L.P., Kamo, S.L., Williams, I.S., Mundil, R., Davis, D.W., Korsch, R.J., and Foudoulis, C., 2003. The application of SHRIMP to Phanerozoic geochronology; a critical appraisal of four zircon standards: Chemical Geology, v. 200, p. 171-188. doi:org/10.1016/S00092541(03)00166-9.

Chen, J. L., Xu, J. F., Kang, Z. Q., and Jie, L., 2010, Origin of Cenozoic alkaline potassic volcanic rocks at KonglongXiang, Lhasa terrane, Tibetan Plateau: Products of partial melting of a mafic lower-crustal source?. Chemical Geology, 273(3-4), 286-299.

Elhlou, S., Belousova, E., Griffin, W., Pearson, N., and O'reilly, S., 2006, Trace element and isotopic composition of GJ-red zircon standard by laser ablation: Geochimica et Cosmochimica Acta, v. 70, p. A158, https://doi.org/10.1016/j.gca.2006.06.1383.

Horstwood, M.S., Košler, J., Gehrels, G., Jackson, S.E., McLean, N.M., Paton, C., Pearson, N.J., Sircombe, K., Sylvester, P., Vermeesch, P. and Bowring, J.F., 2016. Community derived standards for LA ICP MS U ( Th ) $\mathrm{Pb}$ geochronology-Uncertainty propagation, age interpretation and data reporting. Geostandards and Geoanalytical Research, 40(3), pp.311332.

Jackson, S. E., Pearson, N. J., Griffin, W. L., and Belousova, E. A., 2004, The application of laser ablation-inductively coupled plasma-mass spectrometry to in situ U-Pb zircon geochronology: Chemical Geology, v. 211, p. 47-69.

Li, X.H., Long, W.G., Li, Q.L., Liu, Y., Zheng, Y.F., Yang, Y.H., Chamberlain, K.R., Wan, D. F., Guo, C.H. and Wang, X.C. (2010). Penglai zircon megacrysts: a potential new working reference material for microbeam determination of $\mathrm{Hf}-\mathrm{O}$ isotopes and $\mathrm{U}-\mathrm{Pb}$ age. Geostandards and Geoanalytical Research 34, 117-134.

Li, X.H., Tang, G.Q., Gong, B., Yang, Y.H., Hou, K.J., Hu, Z.C., Li, Q.L., Liu, Y., and Li, W.X., 2013, Qinghu zircon: A working reference for microbeam analysis of U-Pb age and $\mathrm{Hf}$ and $\mathrm{O}$ isotopes: Chinese Science Bulletin, v. 58, p. 4647-4654, doi.org/10.1007/s11434-013-5932-x.

Liu, C.Z., Wu, F.Y., Wilde, S.A., Yu, L.J., Li, J.L., 2010. Anorthitic plagioclase and pargasitic amphibole in mantle peridotites from Yungbwa ophiolite (southern Tibetan Plateau) formed by hydrous melt metasomatism. Lithos 114, 413-422. 
Liu, Y., Hu, Z., Gao, S., Günther, D., Xu, J., Gao, C. and Chen, H, 2008, In situ analysis of major and trace elements of anhydrous minerals by LA-ICP-MS without applying an internal standard. Chemical Geology, 257(1-2), 34-43.

Liu Y. S., Hu Z. C., Zong K. Q., Gao C. G., Gao S., Xu J. and Chen H. H., 2010, Reappraisement and refinement of zircon $\mathrm{U}-\mathrm{Pb}$ isotope and trace element analyses by LA-ICP-MS. Chinese Sci. Bull. 55, 1535-1546.

Ludwig, K.R., 2012, Isoplot v. 3.75-A GeochronologicalTool- kit for Microsoft Excel: Berkeley Geochronology Center Special Publication 5, 75 p.

Spencer, C.J., Kirkland, C.L., Taylor, R.J.M., 2016. Strategies towards statistically robust interpretations of in situ U-Pb zircon geochronology. Geoscience Frontiers, 7, 581-589.

Spencer CJ, Kirkland CL, Roberts NM, Evans NJ, Liebmann J. Strategies towards robust interpretations of in situ zircon Lu-Hf isotope analyses. Geoscience Frontiers, 2020,11(3):843-53.

Zou, D., Liu, Y., Hu, Z., Gao, S., Zong, K., Xu, R., and Gao, C., 2014, Pyroxenite and peridotite xenoliths from Hexigten, Inner Mongolia: Insights into the Paleo-Asian Ocean subductionrelated melt/fluid-peridotite interaction. Geochimica et Cosmochimica Acta, 140, 435-454. 


\section{TABLES}

Table S1-Zircon U-Pb isotope data for the EARs

\begin{tabular}{|c|c|c|c|c|c|c|c|c|c|c|c|c|}
\hline No & $\mathrm{Pb}$ & $\mathrm{Th}$ & $\mathrm{U}$ & $\mathrm{Th} / \mathrm{U}$ & $\begin{array}{c}{ }^{207} \mathrm{~Pb} /{ }^{235} \mathrm{U} \\
\text { (Ratio) }\end{array}$ & $\pm 2 \sigma$ & $\begin{array}{c}{ }^{206} \mathrm{~Pb} /{ }^{238} \mathrm{U} \\
\text { (Ratio) }\end{array}$ & $\pm 2 \sigma$ & $\begin{array}{c}{ }^{207} \mathrm{~Pb} /{ }^{2} \\
{ }^{35} \mathrm{U} \\
(\mathrm{Ma})\end{array}$ & $\pm 2 \sigma$ & $\begin{array}{c}{ }^{206} \mathrm{~Pb}^{23} \\
{ }^{8} \mathrm{U} \\
(\mathrm{Ma})\end{array}$ & $\pm 2 \sigma$ \\
\hline \multicolumn{13}{|c|}{$06 \mathrm{DYH}-19:$ Mean $=39.4 \pm 0.6 \mathrm{Ma}, \mathrm{N}=16,($ spot 05 is not used $) \mathrm{MSWD}=1.6$} \\
\hline 01 & 15 & 491 & 1072 & 0.46 & 0.04223 & 0.00278 & 0.00637 & 0.00012 & 42.0 & 2.7 & 40.9 & 0.8 \\
\hline 02 & 16 & 434 & 1267 & 0.34 & 0.04089 & 0.00275 & 0.00620 & 0.00011 & 40.7 & 2.7 & 39.9 & 0.7 \\
\hline 03 & 7 & 201 & 586 & 0.34 & 0.04620 & 0.00346 & 0.00609 & 0.00014 & 45.9 & 3.4 & 39.1 & 0.9 \\
\hline 04 & 9 & 217 & 782 & 0.28 & 0.04189 & 0.00273 & 0.00617 & 0.00011 & 41.7 & 2.7 & 39.6 & 0.7 \\
\hline 05 & 17 & 382 & 1098 & 0.35 & 0.06765 & 0.00407 & 0.00616 & 0.00012 & 66.5 & 3.9 & 39.6 & 0.8 \\
\hline 06 & 35 & 1577 & 1966 & 0.80 & 0.04072 & 0.00268 & 0.00613 & 0.00010 & 40.5 & 2.6 & 39.4 & 0.6 \\
\hline 07 & 21 & 717 & 1584 & 0.45 & 0.03908 & 0.00248 & 0.00607 & 0.00011 & 38.9 & 2.4 & 39.0 & 0.7 \\
\hline 08 & 25 & 976 & 1692 & 0.58 & 0.04033 & 0.00246 & 0.00602 & 0.00009 & 40.2 & 2.4 & 38.7 & 0.6 \\
\hline 09 & 17 & 535 & 1339 & 0.40 & 0.03813 & 0.00244 & 0.00633 & 0.00011 & 38.0 & 2.4 & 40.6 & 0.7 \\
\hline 10 & 46 & 1862 & 2770 & 0.67 & 0.0379 & 0.0026 & 0.0062 & 0.0001 & 37.8 & 2.5 & 39.9 & 0.8 \\
\hline 11 & 21 & 740 & 1387 & 0.53 & 0.04269 & 0.00255 & 0.00644 & 0.00010 & 42.4 & 2.5 & 41.4 & 0.7 \\
\hline 12 & 18 & 565 & 1297 & 0.44 & 0.04270 & 0.00290 & 0.00621 & 0.00011 & 42.5 & 2.8 & 39.9 & 0.7 \\
\hline 13 & 22 & 705 & 1611 & 0.44 & 0.03875 & 0.00247 & 0.00602 & 0.00011 & 38.6 & 2.4 & 38.7 & 0.7 \\
\hline 14 & 28 & 969 & 2044 & 0.47 & 0.03863 & 0.00233 & 0.00592 & 0.00009 & 38.5 & 2.3 & 38.1 & 0.6 \\
\hline 15 & 12 & 331 & 857 & 0.39 & 0.04081 & 0.00270 & 0.00641 & 0.00013 & 40.6 & 2.6 & 41.2 & 0.8 \\
\hline 16 & 14 & 322 & 1291 & 0.25 & 0.03900 & 0.00261 & 0.00618 & 0.00010 & 38.8 & 2.6 & 39.7 & 0.7 \\
\hline 17 & 20 & 650 & 1556 & 0.42 & 0.03919 & 0.00243 & 0.00618 & 0.00010 & 39.0 & 2.4 & 39.7 & 0.6 \\
\hline \multicolumn{13}{|c|}{ LQS-01: Mean $=39.3 \pm 0.4 \mathrm{Ma}, n=16, \mathrm{MSWD}=1.1$} \\
\hline 01 & 10.2 & 488 & 270 & 1.81 & 0.0379 & 0.0026 & 0.0062 & 0.0001 & 41.2 & 2.7 & 38.8 & 0.7 \\
\hline 02 & 15 & 73 & 179 & 0.41 & 0.27379 & 0.01692 & 0.03669 & 0.00077 & 246 & 13.5 & 232.3 & 4.8 \\
\hline 03 & 15 & 390 & 1211 & 0.32 & 0.04165 & 0.00266 & 0.00633 & 0.00011 & 41.4 & 2.6 & 40.7 & 0.7 \\
\hline 04 & 90 & 674 & 941 & 0.72 & 0.20650 & 0.00811 & 0.03314 & 0.00053 & 191 & 6.8 & 210 & 3.3 \\
\hline 05 & 22 & 880 & 1422 & 0.62 & 0.04067 & 0.00248 & 0.00616 & 0.00010 & 40.5 & 2.4 & 39.6 & 0.6 \\
\hline 06 & 10 & 242 & 945 & 0.26 & 0.03820 & 0.00265 & 0.00597 & 0.00010 & 38.1 & 2.6 & 38.4 & 0.6 \\
\hline 07 & 21 & 431 & 2118 & 0.20 & 0.03879 & 0.00192 & 0.00617 & 0.00011 & 38.6 & 1.9 & 39.6 & 0.7 \\
\hline 08 & 8 & 93 & 890 & 0.10 & 0.04466 & 0.00333 & 0.00627 & 0.00012 & 44.4 & 3.2 & 40.3 & 0.8 \\
\hline 09 & 15 & 332 & 1524 & 0.22 & 0.03808 & 0.00240 & 0.00602 & 0.00011 & 37.9 & 2.3 & 38.7 & 2.9 \\
\hline 10 & 18 & 523 & 1507 & 0.35 & 0.04524 & 0.00271 & 0.00638 & 0.00010 & 44.9 & 2.6 & 41.0 & 3.1 \\
\hline 11 & 47 & 1960 & 2807 & 0.70 & 0.03670 & 0.00172 & 0.00595 & 0.00008 & 36.6 & 1.7 & 38.2 & 3.7 \\
\hline 12 & 18 & 539 & 1346 & 0.40 & 0.04325 & 0.00248 & 0.00623 & 0.00011 & 43.0 & 2.4 & 40.0 & 0.7 \\
\hline 13 & 7 & 86 & 805 & 0.11 & 0.04178 & 0.00281 & 0.00613 & 0.00009 & 41.6 & 2.7 & 39.4 & 0.6 \\
\hline 14 & 21 & 134 & 263 & 0.51 & 0.22023 & 0.01118 & 0.03341 & 0.00052 & 202 & 9.3 & 212 & 3.2 \\
\hline 16 & 14 & 313 & 1346 & 0.23 & 0.04095 & 0.00255 & 0.00607 & 0.00011 & 40.8 & 2.5 & 39.0 & 0.7 \\
\hline 17 & 12 & 476 & 768 & 0.62 & 0.04338 & 0.00268 & 0.00614 & 0.00009 & 43.1 & 2.6 & 39.5 & 0.6 \\
\hline 18 & 18 & 262 & 2125 & 0.12 & 0.04111 & 0.00244 & 0.00591 & 0.00009 & 40.9 & 2.4 & 38.0 & 0.6 \\
\hline 19 & 6 & 143 & 658 & 0.22 & 0.03925 & 0.00253 & 0.00599 & 0.00011 & 39.1 & 2.5 & 38.5 & 0.7 \\
\hline 20 & 23 & 590 & 1975 & 0.30 & 0.04308 & 0.00279 & 0.00608 & 0.00012 & 42.8 & 2.7 & 39.1 & 0.7 \\
\hline \multicolumn{13}{|c|}{$06 Z Z-12:$ Mean $=42.3 \pm 1.3 \mathrm{Ma}, n=4, M S W D=1.6$} \\
\hline 01 & 29 & 216 & 293 & 0.74 & 0.24968 & 0.01329 & 0.03353 & 0.00061 & 226 & 10.8 & 213 & 3.8 \\
\hline 02 & 121 & 879 & 1113 & 0.79 & 0.22004 & 0.00947 & 0.03485 & 0.00047 & 202 & 7.9 & 221 & 3.0 \\
\hline 03 & 66 & 455 & 685 & 0.66 & 0.24611 & 0.01218 & 0.03561 & 0.00059 & 223 & 9.9 & 226 & 3.7 \\
\hline 04 & 47 & 1302 & 3842 & 0.34 & 0.04234 & 0.00219 & 0.00602 & 0.00012 & 38.7 & 0.8 & 43.3 & 2.0 \\
\hline 05 & 11 & 379 & 772 & 0.49 & 0.04095 & 0.00227 & 0.00648 & 0.00012 & 40.8 & 2.2 & 41.6 & 0.8 \\
\hline 06 & 52 & 1613 & 4178 & 0.39 & 0.03968 & 0.00255 & 0.00585 & 0.00011 & 37.6 & 0.7 & 41.1 & 2.0 \\
\hline 08 & 91 & 2678 & 6911 & 0.39 & 0.03834 & 0.00205 & 0.00623 & 0.00012 & 40.0 & 0.8 & 45.2 & 1.6 \\
\hline
\end{tabular}




\begin{tabular}{|c|c|c|c|c|c|c|c|c|c|c|c|c|}
\hline \multicolumn{13}{|c|}{$06 T P H-10:$ Mean $=41.5 \pm 0.3 \mathrm{Ma}, n=16($ spots 15 and 17 are not used), $M S W D=1.7$} \\
\hline 01 & 19 & 432 & 1727 & 0.25 & 0.03995 & 0.00201 & 0.00607 & 0.00011 & 39.0 & 0.7 & 41.8 & 2.1 \\
\hline 02 & 16 & 337 & 1480 & 0.23 & 0.04159 & 0.00261 & 0.00644 & 0.00011 & 41.4 & 2.5 & 41.4 & 0.7 \\
\hline 03 & 18 & 412 & 1706 & 0.24 & 0.04418 & 0.00222 & 0.006662 & 0.00009 & 43.9 & 2.2 & 42.8 & 0.6 \\
\hline 04 & 24 & 648 & 2252 & 0.29 & 0.04219 & 0.00229 & 0.00641 & 0.00011 & 42.0 & 2.2 & 41.2 & 0.7 \\
\hline 05 & 28.5 & 654 & 2739 & 0.24 & 0.04354 & 0.00294 & 0.00650 & 0.000122 & 43.3 & 2.9 & 41.8 & 0.8 \\
\hline 06 & 22 & 421 & 1985 & 0.21 & 0.04316 & 0.00302 & 0.00660 & 0.00013 & 42.9 & 2.9 & 42.4 & 0.8 \\
\hline 07 & 27.3 & 391 & 2805 & 0.14 & 0.04273 & 0.00299 & 0.00632 & 0.000124 & 42.5 & 2.9 & 40.6 & 0.8 \\
\hline 08 & 33.0 & 985 & 2216 & 0.44 & 0.04513 & 0.00128 & 0.00634 & 0.00006 & 44.8 & 1.2 & 40.8 & 0.4 \\
\hline 09 & 20 & 398 & 2004 & 0.20 & 0.04316 & 0.00302 & 0.00660 & 0.00013 & 42.9 & 2.9 & 42.4 & 0.8 \\
\hline 10 & 24.0 & 482 & 2314 & 0.21 & 0.04741 & 0.00149 & 0.00630 & 0.00006 & 47.0 & 1.4 & 40.5 & 0.4 \\
\hline 11 & 23.5 & 610 & 2331 & 0.26 & 0.04527 & 0.00265 & 0.00636 & 0.00013 & 45.0 & 2.6 & 40.9 & 0.8 \\
\hline 12 & 23 & 482 & 2436 & 0.20 & 0.04323 & 0.00202 & 0.00629 & 0.00009 & 43.0 & 2.0 & 40.4 & 0.6 \\
\hline 13 & 17 & 339 & 1785 & 0.19 & 0.04417 & 0.00219 & 0.00666 & 0.00009 & 43.9 & 2.1 & 42.8 & 0.6 \\
\hline 14 & 28.4 & 624 & 2538 & 0.25 & 0.04491 & 0.00222 & 0.00662 & 0.00010 & 44.6 & 2.2 & 42.5 & 0.6 \\
\hline 15 & 39.7 & 1109 & 3239 & 0.34 & 0.04336 & 0.00211 & 0.00617 & 0.00009 & 43.1 & 2.1 & 39.7 & 0.6 \\
\hline 16 & 19.9 & 396 & 1918 & 0.21 & 0.04045 & 0.00224 & 0.00632 & 0.00011 & 40.3 & 2.2 & 40.6 & 0.7 \\
\hline 17 & 47 & 1302 & 3842 & 0.34 & 0.04064 & 0.00293 & 0.00684 & 0.00018 & 40.5 & 2.9 & 43.9 & 1.2 \\
\hline 18 & 91 & 2678 & 6911 & 0.39 & 0.03834 & 0.00205 & 0.00623 & 0.00012 & 38.7 & 0.8 & 43.3 & 2.0 \\
\hline \multicolumn{13}{|c|}{ LQS-11: Mean $=39.8 \pm 0.3 \mathrm{Ma}, n=18, M S W D=1.5$} \\
\hline 01 & 44 & 1798 & 2626 & 0.68 & 0.04011 & 0.00261 & 0.00615 & 0.00011 & 39.9 & 2.5 & 39.5 & 0.7 \\
\hline 02 & 20 & 775 & 1338 & 0.58 & 0.04495 & 0.00407 & 0.00624 & 0.00011 & 44.6 & 4.0 & 40.1 & 0.7 \\
\hline 03 & 23 & 615 & 1952 & 0.32 & 0.03728 & 0.00168 & 0.00605 & 0.00008 & 37.2 & 1.6 & 38.9 & 0.5 \\
\hline 05 & 18 & 553 & 1311 & 0.42 & 0.03186 & 0.00778 & 0.00631 & 0.00013 & 31.8 & 7.7 & 40.5 & 0.8 \\
\hline 06 & 7 & 105 & 834 & 0.13 & 0.04262 & 0.00230 & 0.00602 & 0.00008 & 42.4 & 2.2 & 38.7 & 0.5 \\
\hline 07 & 7 & 155 & 677 & 0.23 & 0.03622 & 0.00231 & 0.00611 & 0.00010 & 36.1 & 2.3 & 39.3 & 0.7 \\
\hline 08 & 22 & 705 & 1664 & 0.42 & 0.04175 & 0.00218 & 0.00608 & 0.00009 & 41.5 & 2.1 & 39.1 & 0.6 \\
\hline 09 & 20 & 389 & 1954 & 0.20 & 0.03735 & 0.00201 & 0.00638 & 0.00010 & 37.2 & 2.0 & 41.0 & 0.7 \\
\hline 10 & 8 & 96 & 855 & 0.11 & 0.04022 & 0.00293 & 0.00610 & 0.00011 & 40.0 & 2.9 & 39.2 & 0.7 \\
\hline 12 & 45 & 1808 & 2776 & 0.65 & 0.03946 & 0.00270 & 0.00632 & 0.00013 & 39.3 & 2.6 & 40.6 & 0.8 \\
\hline 13 & 16 & 367 & 1589 & 0.23 & 0.03928 & 0.00272 & 0.00614 & 0.00011 & 39.1 & 2.7 & 39.5 & 0.7 \\
\hline 14 & 15 & 396 & 1256 & 0.32 & 0.04204 & 0.00227 & 0.00629 & 0.00009 & 41.8 & 2.2 & 40.4 & 0.6 \\
\hline 15 & 12 & 479 & 784 & 0.61 & 0.04308 & 0.00279 & 0.00608 & 0.00012 & 42.8 & 2.7 & 39.1 & 0.7 \\
\hline 16 & 10 & 233 & 909 & 0.26 & 0.04030 & 0.00331 & 0.00644 & 0.00013 & 40.1 & 3.2 & 41.4 & 0.8 \\
\hline 17 & 14 & 454 & 1015 & 0.45 & 0.04169 & 0.00232 & 0.00632 & 0.00010 & 41.5 & 2.3 & 40.6 & 0.7 \\
\hline 18 & 15 & 411 & 1206 & 0.34 & 0.04036 & 0.00233 & 0.00631 & 0.00009 & 40.2 & 2.3 & 40.6 & 0.6 \\
\hline 19 & 11 & 322 & 806 & 0.40 & 0.04464 & 0.00276 & 0.00629 & 0.00011 & 44.3 & 2.7 & 40.4 & 0.7 \\
\hline 20 & 13 & 313 & 1245 & 0.25 & 0.04423 & 0.00245 & 0.00618 & 0.00009 & 43.9 & 2.4 & 39.7 & 0.6 \\
\hline \multicolumn{13}{|c|}{ GJ-1: $M e a n=605 \pm 3 \mathrm{Ma}, n=24, M S W D=0.36$} \\
\hline $\begin{array}{c}\text { GJ-1- } \\
01 \\
\end{array}$ & 26.0 & 8.2 & 325 & 0.03 & 0.81006 & 0.01501 & 0.09852 & 0.00127 & 602 & 8 & 606 & 7 \\
\hline $\begin{array}{c}\text { GJ-1- } \\
02\end{array}$ & 26.6 & 8.6 & 337 & 0.03 & 0.81119 & 0.01491 & 0.09876 & 0.00126 & 603 & 8 & 607 & 7 \\
\hline $\begin{array}{c}\text { GJ-1- } \\
03 \\
\end{array}$ & 26.4 & 8.5 & 331 & 0.03 & 0.81232 & 0.01605 & 0.09878 & 0.00116 & 604 & 9 & 607 & 7 \\
\hline $\begin{array}{c}\text { GJ-1- } \\
04\end{array}$ & 26.5 & 8.6 & 332 & 0.03 & 0.80935 & 0.01564 & 0.09825 & 0.00117 & 602 & 9 & 604 & 7 \\
\hline $\begin{array}{c}\text { GJ-1- } \\
05\end{array}$ & 26.4 & 8.6 & 333 & 0.03 & 0.79249 & 0.01533 & 0.09827 & 0.00110 & 593 & 9 & 604 & 6 \\
\hline $\begin{array}{c}\text { GJ-1- } \\
06\end{array}$ & 25.7 & 8.4 & 332 & 0.03 & 0.81856 & 0.01755 & 0.09898 & 0.00103 & 607 & 10 & 608 & 6 \\
\hline $\begin{array}{c}\text { GJ-1- } \\
07\end{array}$ & 25.7 & 8.3 & 331 & 0.03 & 0.80137 & 0.01731 & 0.09829 & 0.00116 & 598 & 10 & 604 & 7 \\
\hline $\begin{array}{c}\text { GJ-1- } \\
08\end{array}$ & 26.3 & 8.4 & 336 & 0.03 & 0.79961 & 0.01624 & 0.09782 & 0.00124 & 597 & 9 & 602 & 7 \\
\hline $\begin{array}{c}\text { GJ-1- } \\
09\end{array}$ & 27.3 & 8.6 & 343 & 0.03 & 0.79364 & 0.01535 & 0.09807 & 0.00117 & 593 & 9 & 603 & 7 \\
\hline $\begin{array}{c}\text { GJ-1- } \\
10\end{array}$ & 27.9 & 9.2 & 310 & 0.03 & 0.80009 & 0.01803 & 0.09839 & 0.00105 & 597 & 10 & 605 & 6 \\
\hline $\begin{array}{c}\text { GJ-1- } \\
11 \\
\end{array}$ & 26.6 & 9.1 & 307 & 0.03 & 0.80631 & 0.01729 & 0.09863 & 0.00106 & 600 & 10 & 606 & 6 \\
\hline
\end{tabular}




\begin{tabular}{|c|c|c|c|c|c|c|c|c|c|c|c|c|}
\hline $\begin{array}{c}\text { GJ-1- } \\
12\end{array}$ & 24.3 & 9.4 & 317 & 0.03 & 0.81363 & 0.01612 & 0.09817 & 0.00122 & 604 & 9 & 604 & 7 \\
\hline $\begin{array}{c}\text { GJ-1- } \\
13\end{array}$ & 24.0 & 9.3 & 317 & 0.03 & 0.79579 & 0.01691 & 0.09792 & 0.00115 & 594 & 10 & 602 & 7 \\
\hline $\begin{array}{c}\text { GJ-1- } \\
14\end{array}$ & 25.4 & 9.6 & 325 & 0.03 & 0.78549 & 0.01656 & 0.09788 & 0.00122 & 589 & 9 & 602 & 7 \\
\hline $\begin{array}{c}\text { GJ-1- } \\
15\end{array}$ & 25.1 & 8.1 & 321 & 0.03 & 0.79396 & 0.01593 & 0.09855 & 0.00121 & 593 & 9 & 606 & 7 \\
\hline $\begin{array}{c}\text { GJ-1- } \\
16\end{array}$ & 25.7 & 8.3 & 329 & 0.03 & 0.80362 & 0.01671 & 0.09766 & 0.00114 & 599 & 9 & 601 & 7 \\
\hline $\begin{array}{c}\text { GJ-1- } \\
17\end{array}$ & 29.8 & 8.3 & 335 & 0.02 & 0.80940 & 0.01809 & 0.09850 & 0.00136 & 602 & 10 & 606 & 8 \\
\hline $\begin{array}{c}\text { GJ-1- } \\
18\end{array}$ & 26.4 & 7.7 & 314 & 0.02 & 0.80934 & 0.02385 & 0.09810 & 0.00168 & 602 & 13 & 603 & 10 \\
\hline $\begin{array}{c}\text { GJ-1- } \\
19\end{array}$ & 26.1 & 8.2 & 327 & 0.03 & 0.81119 & 0.01491 & 0.09876 & 0.00126 & 603 & 8 & 607 & 7 \\
\hline $\begin{array}{c}\text { GJ-1- } \\
20\end{array}$ & 26.5 & 8.5 & 333 & 0.03 & 0.81232 & 0.01605 & 0.09878 & 0.00116 & 604 & 9 & 607 & 7 \\
\hline $\begin{array}{c}\text { GJ-1- } \\
21\end{array}$ & 26.3 & 8.5 & 332 & 0.03 & 0.80935 & 0.01564 & 0.09825 & 0.00117 & 602 & 9 & 604 & 7 \\
\hline $\begin{array}{c}\text { GJ-1- } \\
22\end{array}$ & 26.6 & 8.5 & 333 & 0.03 & 0.79249 & 0.01533 & 0.09827 & 0.00110 & 593 & 9 & 604 & 6 \\
\hline $\begin{array}{c}\text { GJ-1- } \\
23\end{array}$ & 26.3 & 8.4 & 328 & 0.03 & 0.81856 & 0.01755 & 0.09898 & 0.00103 & 607 & 10 & 608 & 6 \\
\hline $\begin{array}{c}\text { GJ-1- } \\
24\end{array}$ & 28.2 & 8.5 & 332 & 0.03 & 0.80137 & 0.01731 & 0.09829 & 0.00116 & 598 & 10 & 604 & 7 \\
\hline
\end{tabular}


Table S2-Lu-Hf and O isotope data for zircon grains from the EARs

\begin{tabular}{|c|c|c|c|c|c|c|c|c|c|c|c|c|}
\hline Sample & ${ }^{176} \mathrm{Yb} /{ }^{177} \mathrm{Hf}$ & $\begin{array}{c}{ }^{176} \mathrm{Lu} /{ }^{177} \\
\mathrm{Hf}\end{array}$ & $\begin{array}{c}{ }^{180} \mathrm{Hf} /{ }^{177} \\
\mathrm{H}\end{array}$ & $\pm 2 \sigma$ & $\begin{array}{c}{ }^{178} \mathrm{Hf} /{ }^{177} \\
\mathrm{H}\end{array}$ & $\pm 2 \sigma$ & ${ }^{176} \mathrm{Hf} /{ }^{177} \mathrm{Hf}$ & $\pm 2 \sigma$ & $\left({ }^{176} \mathrm{Hf} /{ }^{177} \mathrm{Hf}\right)_{\mathrm{i}}$ & $\begin{array}{c}\varepsilon_{\mathrm{Hff}}(\mathrm{t} \\
)\end{array}$ & $\delta^{18} \mathrm{O}$ & $\pm 2 \sigma$ \\
\hline \multicolumn{13}{|c|}{$06 \mathrm{DYH}-19$ Dacite $(\mathrm{SiO} 2=63.1 \mathrm{wt} \% ; \mathrm{Mg \#}=61.8)$} \\
\hline @01 & 0.015 & 0.001 & 1.88721 & 0.00024 & 1.46724 & 0.00033 & 0.282756 & 0.000021 & 0.282756 & 0.3 & 5.3 & 0.2 \\
\hline (a)02 & 0.019 & 0.001 & 1.88644 & 0.00027 & 1.46695 & 0.0002 & 0.282732 & 0.000013 & 0.282731 & -0.6 & 5.6 & 0.2 \\
\hline (a) 03 & 0.026 & 0.001 & 1.88648 & 0.00028 & 1.46694 & 0.00024 & 0.282727 & 0.000027 & 0.282727 & -0.7 & 5.3 & 0.3 \\
\hline @ 04 & 0.015 & 0.001 & 1.88682 & 0.00022 & 1.46713 & 0.00029 & 0.282813 & 0.000021 & 0.282813 & 2.2 & 5.2 & 0.3 \\
\hline @05 & 0.016 & 0.001 & 1.88722 & 0.00024 & 1.46736 & 0.00028 & 0.282756 & 0.000024 & 0.282755 & 0.3 & 5.8 & 0.2 \\
\hline @06 & 0.020 & 0.001 & 1.88694 & 0.00023 & 1.46717 & 0.00025 & 0.282729 & 0.000026 & 0.282728 & -0.7 & 6.0 & 0.2 \\
\hline @07 & 0.021 & 0.001 & 1.88686 & 0.00024 & 1.46721 & 0.00016 & 0.282733 & 0.000043 & 0.282732 & -0.5 & 6.1 & 0.2 \\
\hline @08 & 0.012 & 0.000 & 1.88676 & 0.0002 & 1.46692 & 0.00025 & 0.282780 & 0.000033 & 0.282780 & 1.1 & 5.6 & 0.2 \\
\hline @09 & 0.023 & 0.001 & 1.88704 & 0.00021 & 1.46701 & 0.00015 & 0.282694 & 0.000015 & 0.282694 & -1.9 & 6.2 & 0.2 \\
\hline @ 10 & 0.021 & 0.001 & 1.88650 & 0.00016 & 1.46724 & 0.00024 & 0.282799 & 0.000012 & 0.282798 & 1.9 & 5.6 & 0.2 \\
\hline (a) 11 & 0.019 & 0.001 & 1.88771 & 0.0002 & 1.46742 & 0.00024 & 0.282697 & 0.000014 & 0.282697 & -1.8 & 5.9 & 0.2 \\
\hline (a) 12 & 0.010 & 0.000 & 1.88679 & 0.00032 & 1.46718 & 0.00022 & 0.282688 & 0.000019 & 0.282688 & -2.2 & 5.9 & 0.3 \\
\hline (a) 13 & 0.022 & 0.001 & 1.88623 & 0.00023 & 1.46764 & 0.00027 & 0.282695 & 0.000027 & 0.282695 & -1.9 & 6.3 & 0.2 \\
\hline$@ 14$ & 0.009 & 0.000 & 1.88790 & 0.00018 & 1.46723 & 0.00032 & 0.282685 & 0.000025 & 0.282685 & -2.2 & 6.5 & 0.2 \\
\hline (a)15 & 0.020 & 0.001 & 1.88627 & 0.0003 & 1.46671 & 0.00026 & 0.282707 & 0.000021 & 0.282706 & -1.5 & 6.1 & 0.3 \\
\hline @16 & 0.010 & 0.000 & 1.88563 & 0.00025 & 1.46656 & 0.00018 & 0.282719 & 0.000024 & 0.282719 & -1.0 & 6.1 & 0.2 \\
\hline @ 17 & 0.019 & 0.001 & 1.88665 & 0.00024 & 1.46725 & 0.00023 & 0.282718 & 0.000014 & 0.282718 & -1.0 & 6.2 & 0.2 \\
\hline \multicolumn{13}{|c|}{ LQS-01 Andesite $(\mathrm{SiO} 2=62.0 \mathrm{wt} \% ; \mathrm{Mg} \#=65.9)$} \\
\hline @01 & 0.016 & 0.001 & 1.88694 & 0.00027 & 1.46722 & 0.00028 & 0.282760 & 0.000020 & 0.282760 & 0.3 & 5.9 & 0.2 \\
\hline (a) 02 & 0.046 & 0.002 & 1.88695 & 0.00022 & 1.46720 & 0.00026 & 0.282813 & 0.000021 & 0.282811 & 2.3 & 6.3 & 0.3 \\
\hline (a) 03 & 0.011 & 0.000 & 1.88664 & 0.00023 & 1.46675 & 0.00028 & 0.282722 & 0.000017 & 0.282722 & -1.0 & 6.1 & 0.3 \\
\hline @ 04 & 0.014 & 0.001 & 1.88704 & 0.00026 & 1.46724 & 0.00026 & 0.282569 & 0.000015 & 0.282569 & -6.3 & 6.6 & 0.2 \\
\hline @ 05 & 0.012 & 0.001 & 1.88780 & 0.00025 & 1.46749 & 0.00033 & 0.282850 & 0.000012 & 0.282850 & 3.6 & 5.5 & 0.2 \\
\hline @06 & 0.032 & 0.001 & 1.88788 & 0.00024 & 1.46710 & 0.0002 & 0.282782 & 0.000012 & 0.282781 & 1.2 & 5.4 & 0.2 \\
\hline @07 & 0.034 & 0.002 & 1.88797 & 0.0002 & 1.46732 & 0.00018 & 0.282754 & 0.000017 & 0.282753 & 0.2 & 5.9 & 0.2 \\
\hline @08 & 0.043 & 0.002 & 1.88644 & 0.00024 & 1.46713 & 0.00029 & 0.282797 & 0.000018 & 0.282795 & 1.7 & 5.9 & 0.2 \\
\hline$@ 09$ & 0.010 & 0.000 & 1.88730 & 0.0002 & 1.46710 & 0.00025 & 0.282631 & 0.000016 & 0.282631 & -4.1 & 7.6 & 0.2 \\
\hline (a) 10 & 0.020 & 0.001 & 1.88668 & 0.00032 & 1.46719 & 0.00023 & 0.282838 & 0.000016 & 0.282837 & 3.2 & 5.2 & 0.2 \\
\hline (a) 11 & 0.018 & 0.001 & 1.88620 & 0.00024 & 1.46722 & 0.00023 & 0.282850 & 0.000013 & 0.282849 & 3.6 & 6.2 & 0.2 \\
\hline @ 12 & 0.011 & 0.000 & 1.88675 & 0.00021 & 1.46718 & 0.00021 & 0.282775 & 0.000012 & 0.282774 & 1.0 & 6.6 & 0.2 \\
\hline @13 & 0.056 & 0.002 & 1.88722 & 0.00018 & 1.46708 & 0.00021 & 0.282812 & 0.000016 & 0.282810 & 2.2 & 5.7 & 0.2 \\
\hline (a) 14 & 0.036 & 0.002 & 1.88583 & 0.00018 & 1.46681 & 0.00018 & 0.282780 & 0.000015 & 0.282779 & 1.1 & 6.1 & 0.2 \\
\hline @16 & 0.015 & 0.001 & 1.88663 & 0.00029 & 1.46684 & 0.00018 & 0.282845 & 0.000013 & 0.282844 & 3.4 & 5.7 & 0.2 \\
\hline @ 17 & 0.026 & 0.001 & 1.88660 & 0.00026 & 1.46687 & 0.00038 & 0.282861 & 0.000018 & 0.282860 & 4.0 & 5.3 & 0.2 \\
\hline (a) 18 & 0.005 & 0.000 & 1.88701 & 0.00039 & 1.46751 & 0.0003 & 0.282730 & 0.000014 & 0.282729 & -0.6 & 5.4 & 0.3 \\
\hline (a) 19 & 0.023 & 0.001 & 1.88683 & 0.00024 & 1.46739 & 0.0002 & 0.282848 & 0.000015 & 0.282847 & 3.3 & 5.3 & 0.2 \\
\hline (a) 20 & 0.025 & 0.001 & 1.88764 & 0.00031 & 1.46724 & 0.00033 & 0.282801 & 0.000014 & 0.282800 & 1.4 & 5.9 & 0.3 \\
\hline \multicolumn{13}{|c|}{ LQS-11 Rhyolite $($ SiO2 $=71.7 \mathrm{wt} \% ; \mathrm{Mg} \#=37.5)$} \\
\hline (a) 01 & 0.029 & 0.001 & 1.88649 & 0.00028 & 1.46781 & 0.00024 & 0.282828 & 0.000015 & 0.282827 & 3.0 & 5.1 & 0.2 \\
\hline (a) 02 & 0.033 & 0.001 & 1.88720 & 0.00034 & 1.46711 & 0.00019 & 0.282840 & 0.000012 & 0.282839 & 3.3 & 5.2 & 0.3 \\
\hline (a)03 & 0.041 & 0.002 & 1.88756 & 0.00026 & 1.46712 & 0.00029 & 0.282863 & 0.000015 & 0.282862 & 3.9 & 5.6 & 0.2 \\
\hline$@ 05$ & 0.024 & 0.001 & 1.88783 & 0.00026 & 1.46759 & 0.00024 & 0.282840 & 0.000014 & 0.282839 & 3.1 & 6.1 & 0.2 \\
\hline$@ 06$ & 0.040 & 0.002 & 1.88789 & 0.00023 & 1.46716 & 0.00022 & 0.282751 & 0.000018 & 0.282750 & 0.1 & 7.4 & 0.2 \\
\hline @07 & 0.023 & 0.001 & 1.88780 & 0.00039 & 1.46733 & 0.00017 & 0.282819 & 0.000019 & 0.282818 & 2.6 & 5.8 & 0.3 \\
\hline$@ 08$ & 0.002 & 0.000 & 1.88736 & 0.00019 & 1.46716 & 0.00021 & 0.282845 & 0.000015 & 0.282845 & 3.1 & 5.7 & 0.3 \\
\hline (a)09 & 0.047 & 0.002 & 1.88631 & 0.00022 & 1.46728 & 0.00028 & 0.282874 & 0.000013 & 0.282873 & 4.3 & 5.9 & 0.3 \\
\hline (a) 10 & 0.042 & 0.002 & 1.88643 & 0.00035 & 1.46716 & 0.00032 & 0.282769 & 0.000017 & 0.282767 & -0.1 & 6.1 & 0.3 \\
\hline (a)12 & 0.037 & 0.001 & 1.88639 & 0.00021 & 1.46700 & 0.00024 & 0.282715 & 0.000026 & 0.282714 & -1.1 & 6.3 & 0.3 \\
\hline @13 & 0.067 & 0.003 & 1.88692 & 0.00026 & 1.46714 & 0.00026 & 0.282834 & 0.000037 & 0.282832 & 3.1 & 5.1 & 0.3 \\
\hline @14 & 0.023 & 0.001 & 1.88684 & 0.00031 & 1.46773 & 0.00031 & 0.282795 & 0.000014 & 0.282794 & 1.6 & 6.1 & 0.2 \\
\hline (a) 15 & 0.011 & 0.000 & 1.88734 & 0.00025 & 1.46711 & 0.00024 & 0.282789 & 0.000015 & 0.282789 & 1.6 & 5.3 & 0.2 \\
\hline (a)16 & 0.034 & 0.001 & 1.88658 & 0.00023 & 1.46708 & 0.00018 & 0.282595 & 0.000015 & 0.282594 & -5.4 & 7.6 & 0.2 \\
\hline (a) 17 & 0.039 & 0.001 & 1.88673 & 0.00027 & 1.46706 & 0.00023 & 0.282572 & 0.000013 & 0.282571 & -6.1 & 6.1 & 0.3 \\
\hline (a)18 & 0.016 & 0.001 & 1.88828 & 0.00031 & 1.46746 & 0.00026 & 0.282804 & 0.000014 & 0.282803 & 1.9 & 5.6 & 0.2 \\
\hline (a) 19 & 0.023 & 0.001 & 1.88798 & 0.00025 & 1.46743 & 0.00028 & 0.282875 & 0.000015 & 0.282874 & 4.7 & 5.3 & 0.3 \\
\hline (a) 20 & 0.002 & 0.000 & 1.88745 & 0.0003 & 1.46734 & 0.00024 & 0.282829 & 0.000016 & 0.282829 & 2.8 & 5.4 & 0.3 \\
\hline \multicolumn{13}{|c|}{$06 \mathrm{TPH}-10$ Andesite $(\mathrm{SiO} 2=60.2 \mathrm{wt} \% ; \mathrm{Mg} \#=49.1)$} \\
\hline @01 & 0.014 & 0.001 & 1.88576 & 0.00025 & 1.46706 & 0.0002 & 0.282745 & 0.000016 & 0.282745 & 0.0 & 5.6 & 0.2 \\
\hline @ 02 & 0.016 & 0.001 & 1.88595 & 0.00026 & 1.46740 & 0.00018 & 0.282708 & 0.000017 & 0.282707 & -1.6 & 6.4 & 0.3 \\
\hline$@ 03$ & 0.017 & 0.001 & 1.88590 & 0.00028 & 1.46720 & 0.00025 & 0.282652 & 0.000014 & 0.282652 & -3.5 & 7.0 & 0.2 \\
\hline
\end{tabular}




\begin{tabular}{|c|c|c|c|c|c|c|c|c|c|c|c|c|}
\hline @04 & 0.039 & 0.001 & 1.88682 & 0.00023 & 1.46677 & 0.0003 & 0.282676 & 0.000015 & 0.282675 & -2.6 & $\begin{array}{ll}6.7 \\
\end{array}$ & 0.2 \\
\hline$@ 05$ & 0.017 & 0.001 & 1.88644 & 0.00024 & 1.46687 & 0.00027 & 0.282737 & 0.000016 & 0.282736 & -0.4 & 5.6 & 0.2 \\
\hline$@ 06$ & 0.032 & 0.001 & 1.88640 & 0.00021 & 1.46727 & 0.00018 & 0.282648 & 0.000020 & 0.282647 & $\begin{array}{l}-3.5 \\
\end{array}$ & $\begin{array}{l}6.7 \\
\end{array}$ & 0.2 \\
\hline$@ 07$ & 0.021 & 0.001 & 1.88639 & 0.00019 & 1.46744 & 0.0003 & 0.282753 & 0.000016 & 0.282753 & 0.2 & 6.2 & 0.3 \\
\hline$@ 08$ & 0.023 & 0.001 & 1.88710 & 0.00024 & 1.46708 & 0.00027 & 0.282699 & 0.000014 & 0.282699 & -1.7 & 7.1 & 0.2 \\
\hline @09 & 0.018 & 0.001 & 1.88586 & 0.0003 & 1.46672 & 0.00036 & 0.282708 & 0.000015 & 0.282708 & -1.4 & 6.8 & 0.2 \\
\hline @10 & 0.023 & 0.001 & 1.88577 & 0.00028 & 1.46680 & 0.00019 & 0.282694 & 0.000016 & 0.282693 & -1.9 & 6.2 & 0.3 \\
\hline @11 & 0.021 & 0.001 & 1.88719 & 0.00021 & 1.46727 & 0.00029 & 0.282688 & 0.000017 & 0.282687 & -2.2 & 6.7 & 0.1 \\
\hline @12 & 0.029 & 0.001 & 1.88778 & 0.00039 & 1.46747 & 0.00024 & 0.282695 & 0.000015 & 0.282695 & $\begin{array}{l}-2.0 \\
\end{array}$ & 6.1 & 0.3 \\
\hline @13 & 0.026 & 0.001 & 1.88576 & 0.00034 & 1.46706 & 0.00024 & 0.282667 & 0.000014 & 0.282666 & -2.9 & 6.4 & 0.3 \\
\hline @14 & 0.017 & 0.001 & 1.88595 & 0.00021 & 1.46740 & 0.00031 & 0.282665 & 0.000015 & 0.282665 & $\begin{array}{l}-3.0 \\
\end{array}$ & 6.6 & 0.2 \\
\hline @15 & 0.029 & 0.001 & 1.88651 & 0.0002 & 1.46736 & 0.00027 & 0.282677 & 0.000014 & 0.282677 & -2.5 & 6.4 & 0.3 \\
\hline @16 & 0.022 & 0.001 & 1.88740 & 0.00026 & 1.46712 & 0.00028 & 0.282709 & 0.000016 & 0.282709 & -1.4 & 5.9 & 0.2 \\
\hline @17 & 0.035 & 0.001 & 1.88773 & 0.00023 & 1.46740 & 0.00024 & 0.282690 & 0.000019 & 0.282689 & -2.1 & 6.8 & 0.3 \\
\hline @18 & 0.027 & 0.001 & 1.88572 & 0.00024 & 1.46700 & 0.0002 & 0.282696 & 0.000017 & 0.282695 & -1.8 & 6.7 & 0.2 \\
\hline \multicolumn{13}{|c|}{$06 \mathrm{ZZ-12}$ Dacite (SiO2 $=64.1 \mathrm{wt} \% ; \mathrm{Mg} \#=52.6)$} \\
\hline$@ 04$ & 0.024 & 0.001 & 1.88555 & 0.0002 & 1.46748 & 0.00022 & 0.282813 & 0.000016 & 0.282812 & 2.3 & 5.3 & 0.1 \\
\hline$@ 05$ & 0.023 & 0.001 & 1.88665 & 0.00026 & 1.46768 & 0.00021 & 0.282827 & 0.000015 & 0.282826 & 2.8 & 4.7 & 0.2 \\
\hline$@ 06$ & 0.019 & 0.001 & 1.88686 & 0.00028 & 1.46758 & 0.00022 & 0.282808 & 0.000016 & 0.282807 & 2.3 & 5.4 & 0.1 \\
\hline$@ 08$ & 0.024 & 0.001 & 1.88722 & 0.0002 & 1.46767 & 0.00028 & 0.282776 & 0.000014 & 0.282775 & 1.1 & 5.8 & 0.2 \\
\hline \multicolumn{13}{|c|}{ GJ-1 zircon standard grains for $L u-H$ f isotope analyses } \\
\hline GJ-1@01 & 0.007 & 0.000 & 1.88693 & 0.00028 & 1.46719 & 0.00027 & 0.281984 & 0.000015 & & & & \\
\hline GJ-1@02 & 0.007 & 0.000 & 1.88686 & 0.00021 & 1.46709 & 0.00028 & 0.281986 & 0.000016 & & & & \\
\hline GJ-1@03 & 0.007 & 0.000 & 1.88693 & 0.00021 & 1.46725 & 0.00029 & 0.281995 & 0.000016 & & & & \\
\hline GJ-1@04 & 0.008 & 0.000 & 1.88686 & 0.00031 & 1.46703 & 0.00021 & 0.281982 & 0.000017 & & & & \\
\hline GJ-1@05 & 0.009 & 0.000 & 1.88684 & 0.0003 & 1.46733 & 0.00022 & 0.282019 & 0.000016 & & & & \\
\hline GJ-1@06 & 0.009 & 0.000 & 1.88667 & 0.00031 & 1.46714 & 0.00021 & 0.282021 & 0.000017 & & & & \\
\hline GJ-1@07 & 0.008 & 0.000 & 1.88700 & 0.0002 & 1.46720 & 0.00034 & 0.282018 & 0.000019 & & & & \\
\hline GJ-1@08 & 0.009 & 0.000 & 1.88664 & 0.00022 & 1.46725 & 0.0002 & 0.281980 & 0.000019 & & & & \\
\hline GJ-1@09 & 0.009 & 0.000 & 1.88697 & 0.00031 & 1.46718 & 0.00026 & 0.282015 & 0.000019 & & & & \\
\hline GJ-1@10 & 0.008 & 0.000 & 1.88661 & 0.00024 & 1.46726 & 0.00023 & 0.282011 & 0.000018 & & & & \\
\hline GJ-1@11 & 0.008 & 0.000 & 1.88684 & 0.00033 & 1.46712 & 0.00027 & 0.281992 & 0.000016 & & & & \\
\hline GJ-1@12 & 0.007 & 0.000 & 1.88714 & 0.00027 & 1.46721 & 0.00028 & 0.282017 & 0.000018 & & & & \\
\hline GJ-1@13 & 0.008 & 0.000 & 1.88703 & 0.00035 & 1.46722 & 0.00024 & 0.282013 & 0.000017 & & & & \\
\hline GJ-1@14 & 0.008 & 0.000 & 1.88669 & 0.00021 & 1.46729 & 0.00023 & 0.282017 & 0.000020 & & & & \\
\hline GJ-1@15 & 0.007 & 0.000 & 1.88668 & 0.00033 & 1.46710 & 0.00026 & 0.281992 & 0.000016 & & & & \\
\hline GJ-1@16 & 0.007 & 0.000 & 1.88657 & 0.00017 & 1.46721 & 0.00023 & 0.281998 & 0.000017 & & & & \\
\hline GJ-1@17 & 0.007 & 0.000 & 1.88671 & 0.00026 & 1.46715 & 0.00022 & 0.282022 & 0.000016 & & & & \\
\hline GJ-1@18 & 0.007 & 0.000 & 1.88676 & 0.00025 & 1.46727 & 0.00015 & 0.281999 & 0.000016 & & & & \\
\hline GJ-1@19 & 0.007 & 0.000 & 1.88700 & 0.00026 & 1.46703 & 0.00025 & 0.282015 & 0.000017 & & & & \\
\hline GJ-1@20 & 0.007 & 0.000 & 1.88676 & 0.00022 & 1.46723 & 0.00016 & 0.282017 & 0.000014 & & & & \\
\hline GJ-1@21 & 0.007 & 0.000 & 1.88695 & 0.00025 & 1.46697 & 0.00028 & 0.281995 & 0.000015 & & & & \\
\hline GJ-1@22 & 0.008 & 0.000 & 1.88690 & 0.00023 & 1.46690 & 0.00027 & 0.282022 & 0.000016 & & & & \\
\hline GJ-1@23 & 0.008 & 0.000 & 1.88708 & 0.00039 & 1.46694 & 0.0003 & 0.281982 & 0.000016 & & & & \\
\hline GJ-1@24 & 0.008 & 0.000 & 1.88670 & 0.00024 & 1.46718 & 0.00021 & 0.281995 & 0.000017 & & & & \\
\hline GJ-1@25 & 0.007 & 0.000 & 1.88700 & 0.00016 & 1.46714 & 0.0002 & 0.281995 & 0.000017 & & & & \\
\hline GJ-1@26 & 0.007 & 0.000 & 1.88687 & 0.00031 & 1.46717 & 0.00016 & 0.282004 & 0.000018 & & & & \\
\hline GJ-1@27 & 0.008 & 0.000 & 1.88666 & 0.00029 & 1.46727 & 0.00023 & 0.282025 & 0.000018 & & & & \\
\hline GJ-1@28 & 0.007 & 0.000 & 1.88673 & 0.0002 & 1.46714 & 0.00026 & 0.282019 & 0.000019 & & & & \\
\hline GJ-1@29 & 0.008 & 0.000 & 1.88673 & 0.00018 & 1.46714 & 0.00024 & 0.282021 & 0.000016 & & & & \\
\hline GJ-1@30 & 0.008 & 0.000 & 1.88691 & 0.00025 & 1.46695 & 0.0002 & 0.282006 & 0.000014 & & & & \\
\hline GJ-1@31 & 0.008 & 0.000 & 1.88698 & 0.00028 & 1.46705 & 0.00018 & 0.282024 & 0.000016 & & & & \\
\hline GJ-1@32 & 0.007 & 0.000 & 1.88687 & 0.00028 & 1.46717 & 0.00026 & 0.282011 & 0.000017 & & & & \\
\hline \multicolumn{13}{|c|}{ PengLai zircon standard grains for Lu-Hf isotope analyses } \\
\hline Penglai@01 & 0.022 & 0.000 & 1.88639 & 0.00023 & 1.47425 & 0.00019 & 0.282907 & 0.000020 & & & & \\
\hline Penglai@02 & 0.021 & 0.000 & 1.8861 & 0.00018 & 1.47493 & 0.00029 & 0.282869 & 0.000016 & & & & \\
\hline Penglai@03 & 0.019 & 0.000 & 1.88627 & 0.00019 & 1.47485 & 0.00027 & 0.282895 & 0.000018 & & & & \\
\hline Penglai@04 & 0.022 & 0.000 & 1.88579 & 0.00031 & 1.47465 & 0.00022 & 0.282902 & 0.000018 & & & & \\
\hline Penglai@05 & 0.023 & 0.000 & 1.88580 & 0.00024 & 1.47496 & 0.00025 & 0.282903 & 0.000017 & & & & \\
\hline Penglai@06 & 0.023 & 0.000 & 1.88632 & 0.00024 & 1.47478 & 0.00025 & 0.282897 & 0.000016 & & & & \\
\hline Penglai@07 & 0.021 & 0.000 & 1.88616 & 0.00025 & 1.47418 & 0.00023 & 0.282887 & 0.000020 & & & & \\
\hline Penglai@08 & 0.022 & 0.000 & 1.88642 & 0.00019 & 1.47424 & 0.00027 & 0.282896 & 0.000022 & & & & \\
\hline Penglai@09 & 0.023 & 0.000 & 1.88600 & 0.00016 & 1.47393 & 0.00025 & 0.282893 & 0.000024 & & & & \\
\hline Penglai@10 & 0.021 & 0.000 & 1.88603 & 0.00032 & 1.47409 & 0.00026 & 0.282903 & 0.000021 & & & & \\
\hline
\end{tabular}




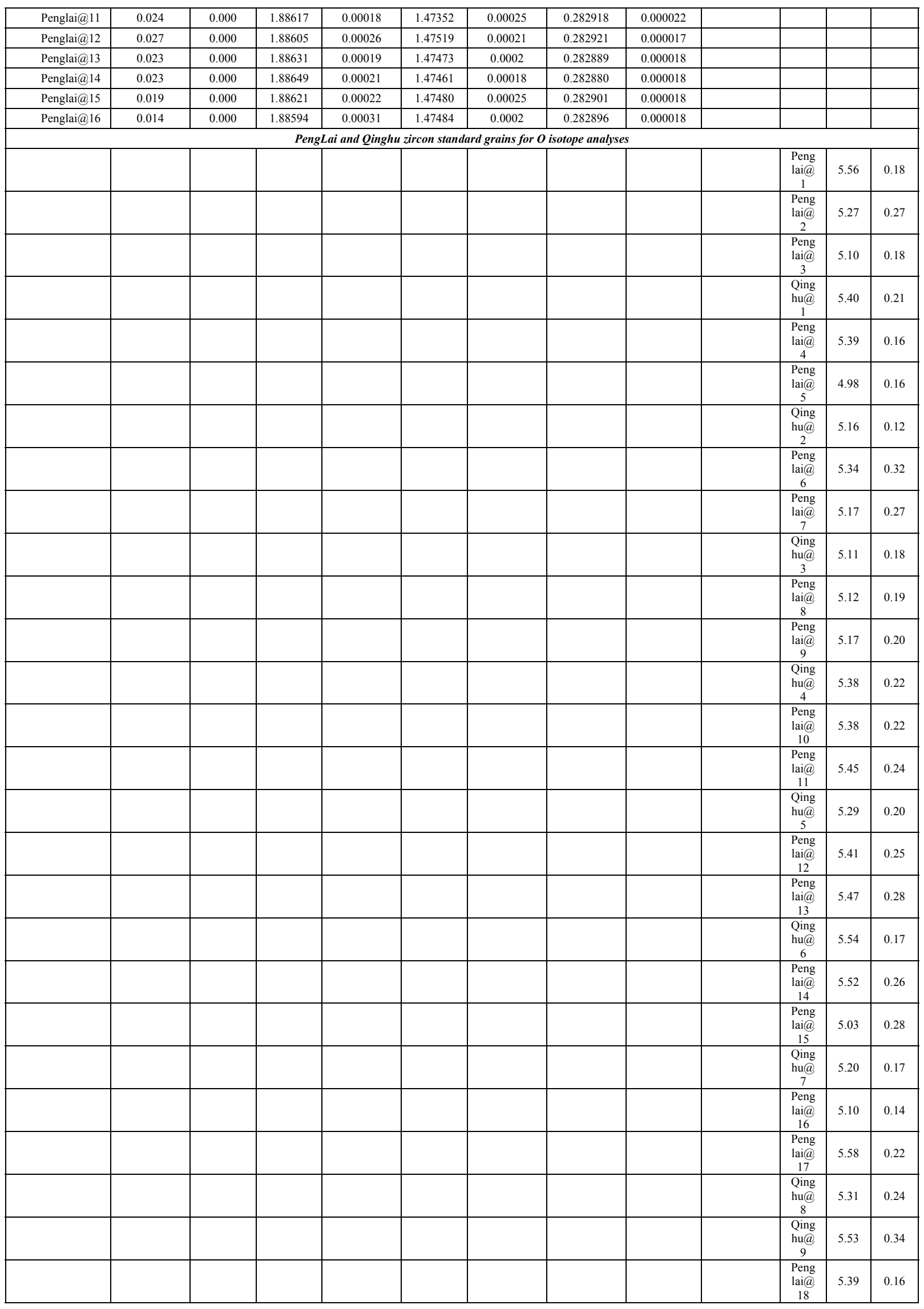




\begin{tabular}{|l|l|l|l|l|l|l|l|l|l|l|l|l|}
\hline & & & & & & & & & & $\begin{array}{c}\text { Peng } \\
\text { lai }(9 \\
\text { 19 }\end{array}$ & 5.29 & 0.28 \\
\hline & & & & & & & & & & $\begin{array}{c}\text { Peng } \\
\text { ani } \\
20\end{array}$ & 5.24 & 0.24 \\
\hline
\end{tabular}

$\varepsilon_{\mathrm{Hf}}(\mathrm{t})=10000 \times\left\{\left[\left({ }^{176} \mathrm{Hf} /{ }^{177} \mathrm{Hf}\right)_{\mathrm{S}}-\left({ }^{176} \mathrm{Lu} /{ }^{177} \mathrm{Hf}\right)_{\mathrm{S}} \times\left(\mathrm{e}^{\lambda \mathrm{t}}-1\right)\right] /\left[\left({ }^{176} \mathrm{Hf} /{ }^{177} \mathrm{Hf}\right)_{\mathrm{CHUR}, 0} \quad-\right.\right.$ $\left.\left.\left({ }^{176} \mathrm{Lu} /{ }^{177} \mathrm{Hf}\right)_{\mathrm{CHUR}} \times\left(\mathrm{e}^{\lambda t}-1\right)\right]-1\right\}$; where $\lambda=1.867 \times 10^{-11}$ year ${ }^{-1}$ (Soderlund et al., 2004), $\left({ }^{176} \mathrm{Lu} /{ }^{177} \mathrm{Hf}\right)_{\mathrm{S}}$ and $\left({ }^{176} \mathrm{Hf} /{ }^{177} \mathrm{Hf}\right)_{\mathrm{S}}$ are measured values from the samples, $\left({ }^{176} \mathrm{Lu} /{ }^{177} \mathrm{Hf}\right)_{\mathrm{CHUR}}=$ 0.0332 and $\left({ }^{176} \mathrm{Hf} /{ }^{177} \mathrm{Hf}\right)_{\mathrm{CHUR}, 0}=0.282772$ (Blichert-Toft and Albarède, 1997).

\section{References}

Blichert-Toft, J. and Albarède, F., 1997, The Lu-Hf geochemistry of chondrites and the evolution of the mantle-crust system. Earth and Planetary Science Letters 148, 24-258.

Soderlund, U., Patchett, P.J., Vervoort, J.D. and Isachsen, C.E., 2004, The ${ }^{176} \mathrm{Lu}$ decay constant determined by $\mathrm{Lu}-\mathrm{Hf}$ and $\mathrm{U}-\mathrm{Pb}$ isotope systematics of Precambrian mafic intrusions. Earth and Planetary Science Letters 219, 311-324. 
Table S3-Bulk-rock major and trace element data for the EARs

\begin{tabular}{|c|c|c|c|c|c|c|c|c|c|c|c|c|}
\hline Sample & $\begin{array}{c}06 \mathrm{DYH} \\
-19\end{array}$ & $\begin{array}{l}06 \mathrm{TPH} \\
-10\end{array}$ & $\begin{array}{l}\text { LQS- } \\
1\end{array}$ & $\begin{array}{l}\text { LQS- } \\
5\end{array}$ & $\begin{array}{l}\text { LQS- } \\
07\end{array}$ & $\begin{array}{l}\text { LQS- } \\
10\end{array}$ & $\begin{array}{l}\text { LQS- } \\
11\end{array}$ & $\begin{array}{l}\text { LQS- } \\
13\end{array}$ & $\begin{array}{l}\text { LQS- } \\
15\end{array}$ & $\begin{array}{l}\text { LQS- } \\
16\end{array}$ & $\begin{array}{l}\text { LQS- } \\
17\end{array}$ & $\begin{array}{l}\text { LQS- } \\
18\end{array}$ \\
\hline Location & Dongyuehu & Taipinghu & \multicolumn{10}{|c|}{ Luanqingshan } \\
\hline $\mathrm{SiO}_{2}$ & 63.10 & 60.16 & 62.02 & 70.96 & 71.56 & 71.58 & 71.67 & 63.12 & 63.84 & 64.40 & 62.71 & 60.07 \\
\hline $\mathrm{TiO}_{2}$ & 0.70 & 0.76 & 0.54 & 0.24 & 0.26 & 0.27 & 0.26 & 0.72 & 0.56 & 0.55 & 0.51 & 0.93 \\
\hline $\mathrm{Al}_{2} \mathrm{O}_{3}$ & 15.67 & 15.30 & 14.75 & 15.68 & 15.13 & 15.14 & 15.28 & 16.58 & 16.01 & 16.26 & 15.73 & 16.29 \\
\hline $\mathrm{Fe}_{2} \mathrm{O}_{3}{ }^{\mathrm{T}}$ & 4.52 & 5.37 & 4.78 & 1.99 & 2.07 & 1.93 & 1.87 & 5.20 & 4.38 & 4.56 & 4.29 & 5.47 \\
\hline $\mathrm{MnO}$ & 0.06 & 0.10 & 0.04 & 0.02 & 0.02 & 0.02 & 0.02 & 0.04 & 0.06 & 0.05 & 0.10 & 0.07 \\
\hline $\mathrm{MgO}$ & 3.66 & 2.59 & 4.63 & 0.66 & 0.62 & 0.67 & 0.79 & 2.88 & 2.73 & 2.26 & 2.21 & 4.22 \\
\hline $\mathrm{CaO}$ & 4.73 & 5.20 & 5.16 & 2.41 & 1.98 & 2.03 & 2.16 & 4.52 & 4.99 & 4.56 & 7.04 & 5.88 \\
\hline $\mathrm{Na}_{2} \mathrm{O}$ & 3.69 & 3.84 & 3.81 & 4.03 & 3.96 & 4.13 & 3.97 & 3.86 & 4.13 & 4.29 & 4.09 & 3.80 \\
\hline $\mathrm{K}_{2} \mathrm{O}$ & 3.46 & 4.13 & 3.62 & 3.73 & 3.94 & 3.85 & 3.92 & 2.59 & 2.70 & 2.75 & 2.62 & 2.75 \\
\hline $\mathrm{P}_{2} \mathrm{O}_{5}$ & 0.28 & 0.54 & 0.33 & 0.03 & 0.01 & 0.01 & 0.03 & 0.23 & 0.15 & 0.03 & 0.16 & 0.25 \\
\hline LOI & 1.35 & 1.02 & 1.53 & 1.26 & 0.90 & 0.65 & 0.72 & 1.45 & 1.42 & 1.05 & 4.59 & 0.65 \\
\hline total & 100.21 & 99.2 & 99.68 & 99.76 & 99.55 & 99.64 & 99.97 & 99.74 & 99.53 & 99.69 & 99.46 & 99.74 \\
\hline $\mathrm{Mg} \#$ & 61.8 & 49.1 & 66.0 & 39.9 & 37.5 & 41.0 & 45.8 & 52.6 & 55.5 & 49.8 & 50.7 & 60.7 \\
\hline $\mathrm{Cr}$ & 143 & 111.7 & 252 & 9.14 & 6.32 & 4.95 & 11.8 & 140 & 95.9 & 88.4 & 110 & 139 \\
\hline $\mathrm{Ni}$ & 90.2 & 55.46 & 134 & 4.24 & 3.97 & 2.90 & 5.02 & 86.9 & 70.1 & 64.5 & 86.9 & 110 \\
\hline $\mathrm{Rb}$ & 119 & 161.9 & 95.9 & 161 & 161 & 152 & 166 & 66.4 & 67.6 & 72.6 & 64.8 & 67.3 \\
\hline $\mathrm{Sr}$ & 1036 & 2260 & 2198 & 432 & 415 & 483 & 454 & 1311 & 1202 & 1248 & 1376 & 1645 \\
\hline $\mathrm{Y}$ & 12.7 & 13.12 & 12.5 & 4.91 & 4.53 & 3.95 & 4.68 & 12.3 & 10.6 & 7.77 & 10.8 & 13.3 \\
\hline $\mathrm{Zr}$ & 255 & 350.6 & 186 & 144 & 145 & 151 & 134 & 208 & 159 & 157 & 144 & 216 \\
\hline $\mathrm{Nb}$ & 10.5 & 14.98 & 7.86 & 3.33 & 3.65 & 3.51 & 4.00 & 7.79 & 5.64 & 5.53 & 5.07 & 14.0 \\
\hline $\mathrm{Ba}$ & 1319 & 1968 & 1903 & 1190 & 1248 & 1259 & 1279 & 1619 & 1374 & 1456 & 1429 & 1383 \\
\hline $\mathrm{La}$ & 57.9 & 109.2 & 77.7 & 21.2 & 29.7 & 31.9 & 30.3 & 47.0 & 27.2 & 18.2 & 26.1 & 48.6 \\
\hline $\mathrm{Ce}$ & 107 & 207.9 & 139 & 36.0 & 51.0 & 54.0 & 51.5 & 84.3 & 48.8 & 30.3 & 48.9 & 91.6 \\
\hline $\operatorname{Pr}$ & 11.6 & 23.65 & 16.1 & 3.94 & 5.34 & 5.64 & 5.49 & 9.71 & 5.80 & 3.43 & 5.77 & 11.2 \\
\hline $\mathrm{Nd}$ & 40.8 & 83.81 & 56.7 & 13.4 & 17.1 & 18.7 & 18.0 & 35.4 & 21.5 & 13.0 & 22.1 & 42.1 \\
\hline $\mathrm{Sm}$ & 5.97 & 12.04 & 7.93 & 2.14 & 2.54 & 2.64 & 2.74 & 5.71 & 3.76 & 2.45 & 3.91 & 6.83 \\
\hline $\mathrm{Eu}$ & 1.46 & 2.85 & 1.91 & 0.49 & 0.51 & 0.55 & 0.53 & 1.38 & 0.99 & 0.88 & 0.95 & 1.61 \\
\hline $\mathrm{Gd}$ & 4.38 & 8.232 & 4.03 & 1.44 & 1.44 & 1.43 & 1.62 & 3.59 & 2.66 & 1.79 & 2.74 & 4.08 \\
\hline $\mathrm{Tb}$ & 0.501 & 0.92 & 0.57 & 0.21 & 0.22 & 0.20 & 0.24 & 0.55 & 0.41 & 0.29 & 0.42 & 0.61 \\
\hline Dy & 2.45 & 4.38 & 2.59 & 1.03 & 0.97 & 0.88 & 1.03 & 2.83 & 2.18 & 1.62 & 2.05 & 2.92 \\
\hline Ho & 0.433 & 0.80 & 0.43 & 0.18 & 0.17 & 0.14 & 0.17 & 0.50 & 0.40 & 0.31 & 0.42 & 0.53 \\
\hline $\mathrm{Er}$ & 1.13 & 2.01 & 1.16 & 0.43 & 0.41 & 0.36 & 0.41 & 1.20 & 1.06 & 0.84 & 1.06 & 1.33 \\
\hline $\mathrm{Tm}$ & 0.156 & 0.28 & 0.16 & 0.07 & 0.06 & 0.04 & 0.06 & 0.17 & 0.16 & 0.14 & 0.17 & 0.18 \\
\hline $\mathrm{Yb}$ & 0.981 & 1.72 & 1.04 & 0.42 & 0.38 & 0.32 & 0.38 & 1.13 & 1.10 & 0.98 & 1.17 & 1.25 \\
\hline $\mathrm{Lu}$ & 0.152 & 0.27 & 0.17 & 0.07 & 0.06 & 0.05 & 0.06 & 0.17 & 0.19 & 0.17 & 0.19 & 0.20 \\
\hline $\mathrm{Hf}$ & 6.44 & 8.74 & 4.46 & 3.96 & 4.00 & 4.14 & 3.88 & 5.13 & 4.21 & 4.20 & 3.81 & 5.30 \\
\hline $\mathrm{Ta}$ & 0.569 & 0.79 & 0.48 & 0.40 & 0.37 & 0.31 & 0.40 & 0.52 & 0.42 & 0.43 & 0.38 & 1.00 \\
\hline Th & 20.0 & 29.94 & 21.1 & 14.5 & 19.3 & 18.5 & 20.6 & 13.8 & 9.48 & 8.73 & 8.24 & 13.3 \\
\hline $\mathrm{U}$ & 4.76 & 6.011 & 3.21 & 5.42 & 5.48 & 5.07 & 6.10 & 2.34 & 3.05 & 2.70 & 2.63 & 3.55 \\
\hline
\end{tabular}

Table S3-continued

\begin{tabular}{|c|c|c|c|c|c|c|c|c|c|c|}
\hline Sample & $\begin{array}{c}\text { LQS- } \\
19 \\
\end{array}$ & $\begin{array}{c}\text { LQS- } \\
20 \\
\end{array}$ & $\begin{array}{c}06 \text { YJL- } \\
01\end{array}$ & $\begin{array}{c}06 \text { YJL- } \\
02 \\
\end{array}$ & $\begin{array}{c}06 \text { YJL- } \\
03 \\
\end{array}$ & $\begin{array}{c}06 \mathrm{YJL}- \\
04 \\
\end{array}$ & $\begin{array}{c}\text { 06YJL- } \\
05 \\
\end{array}$ & $\begin{array}{c}\text { 06YJL- } \\
06 \\
\end{array}$ & $\begin{array}{c}\text { 06YJL- } \\
07 \\
\end{array}$ & $\begin{array}{l}06 \text { YJL- } \\
08\end{array}$ \\
\hline Location & \multicolumn{2}{|c|}{ Luanqingshan } & \multicolumn{8}{|c|}{ Yuejinla } \\
\hline $\mathrm{SiO}_{2}$ & 66.30 & 65.53 & 65.28 & 64.21 & 64.40 & 64.56 & 62.94 & 65.78 & 62.30 & 63.10 \\
\hline $\mathrm{TiO}_{2}$ & 0.54 & 0.59 & 0.70 & 0.80 & 0.66 & 0.70 & 0.75 & 0.71 & 0.74 & 0.77 \\
\hline $\mathrm{Al}_{2} \mathrm{O}_{3}$ & 16.06 & 16.45 & 15.66 & 15.58 & 15.47 & 14.59 & 15.47 & 15.07 & 14.99 & 16.07 \\
\hline $\mathrm{Fe}_{2} \mathrm{O}_{3}{ }^{\mathrm{T}}$ & 3.71 & 3.47 & 3.87 & 4.39 & 4.15 & 4.59 & 4.67 & 4.01 & 4.98 & 4.79 \\
\hline $\mathrm{MnO}$ & 0.05 & 0.05 & 0.06 & 0.08 & 0.06 & 0.07 & 0.06 & 0.05 & 0.07 & 0.05 \\
\hline
\end{tabular}




\begin{tabular}{|c|c|c|c|c|c|c|c|c|c|c|}
\hline $\mathrm{MgO}$ & 2.30 & 2.62 & 2.20 & 2.78 & 3.05 & 3.64 & 3.64 & 2.86 & 4.27 & 3.15 \\
\hline $\mathrm{CaO}$ & 3.66 & 3.72 & 3.46 & 4.39 & 3.88 & 3.57 & 4.27 & 3.27 & 4.38 & 4.00 \\
\hline $\mathrm{Na}_{2} \mathrm{O}$ & 3.92 & 4.03 & 3.41 & 2.84 & 3.09 & 2.55 & 3.07 & 3.12 & 2.79 & 3.28 \\
\hline $\mathrm{K}_{2} \mathrm{O}$ & 3.19 & 3.11 & 4.11 & 3.81 & 4.25 & 4.43 & 4.16 & 4.29 & 4.01 & 4.04 \\
\hline $\mathrm{P}_{2} \mathrm{O}_{5}$ & 0.18 & 0.10 & 0.03 & 0.24 & 0.12 & 0.10 & 0.13 & 0.03 & 0.10 & 0.06 \\
\hline LOI & 1.34 & 2.19 & 0.76 & 1.23 & 1.16 & 1.74 & 1.08 & 0.62 & 1.43 & 1.22 \\
\hline total & 99.9 & 99.7 & 99.5 & 100.4 & 100.3 & 100.5 & 100.3 & 99.8 & 100.1 & 100.5 \\
\hline $\mathrm{Mg} \#$ & 55.4 & 60.2 & 53.2 & 55.9 & 59.5 & 61.3 & 60.9 & 58.8 & 63.2 & 56.8 \\
\hline $\mathrm{Cr}$ & 57.9 & 68.9 & 105 & 102 & 116 & 150 & 139 & 140 & 173 & 135 \\
\hline $\mathrm{Ni}$ & 43.7 & 48.8 & 48.7 & 42.8 & 55.3 & 72.1 & 73.6 & 63.9 & 97.9 & 71.9 \\
\hline $\mathrm{Rb}$ & 83.1 & 74.3 & 158 & 140 & 154 & 183 & 146 & 176 & 171 & 129 \\
\hline $\mathrm{Sr}$ & 1014 & 1148 & 1262 & 1318 & 1121 & 1089 & 1456 & 1118 & 1517 & 1582 \\
\hline $\mathrm{Y}$ & 9.49 & 8.30 & 5.85 & 14.1 & 9.88 & 13.3 & 10.9 & 8.89 & 11.8 & 9.17 \\
\hline $\mathrm{Zr}$ & 253 & 268 & 266 & 257 & 258 & 273 & 269 & 287 & 276 & 284 \\
\hline $\mathrm{Nb}$ & 6.50 & 7.59 & 10.9 & 11.5 & 10.8 & 12.8 & 11.4 & 13.2 & 12.7 & 11.7 \\
\hline $\mathrm{Ba}$ & 1370 & 1517 & 1896 & 1890 & 1847 & 1872 & 2037 & 1888 & 2041 & 2259 \\
\hline $\mathrm{La}$ & 55.4 & 48.7 & 37.2 & 68.8 & 62.0 & 62.7 & 64.7 & 43.6 & 68.1 & 56.9 \\
\hline $\mathrm{Ce}$ & 95.4 & 84.0 & 63.6 & 119 & 104 & 114 & 117 & 76.4 & 122 & 93.0 \\
\hline $\operatorname{Pr}$ & 10.6 & 8.95 & 6.18 & 13.3 & 10.3 & 12.3 & 12.3 & 7.99 & 12.6 & 10.5 \\
\hline $\mathrm{Nd}$ & 36.8 & 30.4 & 20.2 & 46.5 & 34.3 & 42.1 & 41.9 & 26.2 & 43.6 & 36.0 \\
\hline $\mathrm{Sm}$ & 5.38 & 4.38 & 3.02 & 6.55 & 4.83 & 6.02 & 5.89 & 3.75 & 6.30 & 4.90 \\
\hline $\mathrm{Eu}$ & 1.21 & 1.05 & 1.39 & 1.69 & 1.27 & 1.40 & 1.58 & 1.42 & 1.51 & 1.73 \\
\hline $\mathrm{Gd}$ & 3.09 & 2.66 & 1.57 & 3.80 & 2.81 & 3.70 & 3.41 & 2.20 & 3.35 & 2.85 \\
\hline $\mathrm{Tb}$ & 0.44 & 0.37 & 0.25 & 0.55 & 0.43 & 0.54 & 0.49 & 0.34 & 0.49 & 0.42 \\
\hline Dy & 2.06 & 1.82 & 1.22 & 2.85 & 2.03 & 2.70 & 2.34 & 1.84 & 2.47 & 1.95 \\
\hline Ho & 0.36 & 0.32 & 0.22 & 0.53 & 0.37 & 0.49 & 0.41 & 0.34 & 0.42 & 0.35 \\
\hline $\mathrm{Er}$ & 0.91 & 0.83 & 0.59 & 1.34 & 0.94 & 1.29 & 1.09 & 0.88 & 1.16 & 0.92 \\
\hline $\mathrm{Tm}$ & 0.13 & 0.12 & 0.08 & 0.18 & 0.12 & 0.18 & 0.14 & 0.14 & 0.16 & 0.12 \\
\hline $\mathrm{Yb}$ & 0.89 & 0.85 & 0.50 & 1.16 & 0.81 & 1.16 & 0.84 & 0.79 & 1.08 & 0.76 \\
\hline $\mathrm{Lu}$ & 0.15 & 0.13 & 0.08 & 0.19 & 0.12 & 0.19 & 0.14 & 0.14 & 0.16 & 0.12 \\
\hline Hf & 6.01 & 6.59 & 6.16 & 5.86 & 6.02 & 6.44 & 6.31 & 6.73 & 6.49 & 6.70 \\
\hline $\mathrm{Ta}$ & 0.42 & 0.49 & 0.77 & 0.77 & 0.74 & 1.00 & 0.77 & 1.03 & 0.92 & 0.80 \\
\hline Th & 18.7 & 17.5 & 17.3 & 21.5 & 21.5 & 22.4 & 22.4 & 20.0 & 23.6 & 19.6 \\
\hline $\mathrm{U}$ & 3.73 & 3.70 & 4.87 & 5.34 & 5.93 & 6.42 & 5.72 & 6.00 & 6.24 & 5.60 \\
\hline
\end{tabular}

Table S3-continued

\begin{tabular}{|c|c|c|c|c|c|c|c|c|c|c|c|}
\hline Sample & $\begin{array}{l}\text { 06YJL- } \\
09\end{array}$ & $\begin{array}{l}\text { 06YJL- } \\
10\end{array}$ & $\begin{array}{l}\text { 06YJL- } \\
11\end{array}$ & $\begin{array}{l}06 \text { YJL- } \\
12 \\
\end{array}$ & $\begin{array}{l}06 \text { YJL- } \\
13\end{array}$ & $\begin{array}{l}\text { 06YJL- } \\
14\end{array}$ & $\begin{array}{l}\text { 06YJL- } \\
15 \\
\end{array}$ & $\begin{array}{l}06 Y J L- \\
16 \\
\end{array}$ & $\begin{array}{l}\text { 06YJL- } \\
17 \\
\end{array}$ & $\begin{array}{l}06 Y J L- \\
18 \\
\end{array}$ & $\begin{array}{l}06 \text { YJL- } \\
19 \\
\end{array}$ \\
\hline Location & \multicolumn{11}{|c|}{ Yuejinla } \\
\hline $\mathrm{SiO}_{2}$ & 64.20 & 66.73 & 65.83 & 61.18 & 61.67 & 62.10 & 64.81 & 66.82 & 59.23 & 61.30 & 61.97 \\
\hline $\mathrm{TiO}_{2}$ & 0.66 & 0.63 & 0.64 & 0.84 & 0.77 & 0.85 & 0.60 & 0.63 & 0.91 & 0.78 & 0.78 \\
\hline $\mathrm{Al}_{2} \mathrm{O}_{3}$ & 15.23 & 14.96 & 14.85 & 15.23 & 15.22 & 15.44 & 14.92 & 15.74 & 14.50 & 15.09 & 15.22 \\
\hline $\mathrm{Fe}_{2} \mathrm{O}_{3}{ }^{\mathrm{T}}$ & 4.12 & 3.84 & 4.10 & 5.30 & 4.99 & 4.99 & 4.46 & 3.74 & 5.57 & 5.00 & 5.12 \\
\hline $\mathrm{MnO}$ & 0.06 & 0.05 & 0.06 & 0.07 & 0.07 & 0.06 & 0.06 & 0.02 & 0.07 & 0.07 & 0.07 \\
\hline $\mathrm{MgO}$ & 3.10 & 2.35 & 2.87 & 4.23 & 4.25 & 3.79 & 2.46 & 0.63 & 4.88 & 4.20 & 4.16 \\
\hline $\mathrm{CaO}$ & 3.89 & 3.08 & 3.30 & 4.47 & 4.63 & 4.40 & 3.48 & 2.73 & 5.52 & 4.45 & 4.41 \\
\hline $\mathrm{Na}_{2} \mathrm{O}$ & 2.95 & 3.00 & 2.93 & 2.83 & 2.91 & 3.23 & 2.64 & 3.39 & 2.81 & 2.48 & 2.85 \\
\hline
\end{tabular}




\begin{tabular}{|c|c|c|c|c|c|c|c|c|c|c|c|}
\hline $\mathrm{K}_{2} \mathrm{O}$ & 4.28 & 4.38 & 4.36 & 4.42 & 4.20 & 4.29 & 4.65 & 4.55 & 4.71 & 4.54 & 4.47 \\
\hline $\mathrm{P}_{2} \mathrm{O}_{5}$ & 0.15 & 0.09 & 0.04 & 0.11 & 0.15 & 0.04 & 0.25 & 0.17 & 0.37 & 0.09 & 0.08 \\
\hline LOI & 1.34 & 0.71 & 0.73 & 1.30 & 1.16 & 0.64 & 2.10 & 0.98 & 0.96 & 2.03 & 0.81 \\
\hline total & 99.97 & 99.82 & 99.71 & 99.97 & 100.01 & 99.83 & 100.42 & 99.40 & 99.52 & 100.03 & 99.92 \\
\hline $\mathrm{Mg} \#$ & 60.1 & 55.0 & 58.3 & 61.5 & 63.0 & 60.3 & 52.5 & 25.2 & 63.7 & 62.7 & 61.9 \\
\hline $\mathrm{Cr}$ & 123 & 109 & 116 & 170 & 162 & 163 & 77.8 & 55.2 & 230 & 167 & 177 \\
\hline $\mathrm{Ni}$ & 61.4 & 47.5 & 52.8 & 89.4 & 94.1 & 75.3 & 32.6 & 38.2 & 149 & 89.8 & 90.1 \\
\hline $\mathrm{Rb}$ & 162 & 184 & 173 & 160 & 156 & 171 & 176 & 180 & 137 & 171 & 158 \\
\hline $\mathrm{Sr}$ & 1171 & 1204 & 1124 & 1540 & 1628 & 1623 & 1056 & 1153 & 1929 & 1571 & 1538 \\
\hline $\mathrm{Y}$ & 9.96 & 10.6 & 11.7 & 11.2 & 12.0 & 9.08 & 14.6 & 11.5 & 15.5 & 11.5 & 10.5 \\
\hline $\mathrm{Zr}$ & 264 & 270 & 258 & 295 & 271 & 306 & 265 & 289 & 374 & 277 & 276 \\
\hline $\mathrm{Nb}$ & 10.7 & 14.0 & 13.5 & 14.3 & 12.6 & 14.7 & 13.6 & 14.3 & 12.6 & 13.1 & 12.8 \\
\hline $\mathrm{Ba}$ & 1851 & 1727 & 1676 & 1955 & 2056 & 2144 & 1484 & 1709 & 2482 & 2023 & 2035 \\
\hline $\mathrm{La}$ & 62.1 & 53.0 & 70.7 & 67.6 & 71.5 & 47.0 & 78.9 & 67.8 & 72.5 & 69.4 & 64.9 \\
\hline $\mathrm{Ce}$ & 111 & 90.3 & 119 & 116 & 125 & 79.5 & 129 & 112 & 131 & 121 & 111 \\
\hline $\operatorname{Pr}$ & 12.0 & 9.64 & 12.2 & 12.8 & 14.0 & 8.45 & 14.0 & 12.2 & 15.9 & 13.6 & 12.1 \\
\hline $\mathrm{Nd}$ & 40.8 & 33.6 & 38.6 & 42.7 & 48.4 & 30.1 & 46.9 & 40.2 & 58.9 & 46.6 & 41.6 \\
\hline $\mathrm{Sm}$ & 5.65 & 4.95 & 5.37 & 6.02 & 6.70 & 4.65 & 6.77 & 5.61 & 8.99 & 6.29 & 5.76 \\
\hline $\mathrm{Eu}$ & 1.44 & 1.60 & 1.33 & 1.65 & 1.72 & 1.68 & 1.43 & 1.52 & 2.25 & 1.60 & 1.57 \\
\hline $\mathrm{Gd}$ & 3.16 & 2.79 & 2.99 & 3.27 & 3.64 & 2.68 & 3.86 & 3.29 & 4.91 & 3.58 & 3.04 \\
\hline $\mathrm{Tb}$ & 0.45 & 0.44 & 0.47 & 0.48 & 0.53 & 0.40 & 0.60 & 0.49 & 0.67 & 0.50 & 0.46 \\
\hline Dy & 2.14 & 2.20 & 2.34 & 2.34 & 2.48 & 2.01 & 2.81 & 2.36 & 3.12 & 2.45 & 2.22 \\
\hline Но & 0.37 & 0.39 & 0.44 & 0.41 & 0.440 & 0.36 & 0.49 & 0.43 & 0.520 & 0.430 & 0.40 \\
\hline $\mathrm{Er}$ & 0.96 & 1.06 & 1.16 & 1.06 & 1.15 & 0.89 & 1.26 & 1.05 & 1.38 & 1.11 & 1.05 \\
\hline $\mathrm{Tm}$ & 0.13 & 0.15 & 0.17 & 0.14 & 0.15 & 0.13 & 0.19 & 0.16 & 0.190 & 0.150 & 0.14 \\
\hline $\mathrm{Yb}$ & 0.81 & 0.93 & 1.06 & 0.92 & 0.93 & 0.76 & 1.25 & 1.01 & 1.22 & 0.93 & 0.84 \\
\hline $\mathrm{Lu}$ & 0.13 & 0.15 & 0.18 & 0.14 & 0.14 & 0.12 & 0.20 & 0.160 & 0.19 & 0.15 & 0.14 \\
\hline $\mathrm{Hf}$ & 6.21 & 6.44 & 6.46 & 6.77 & 6.21 & 6.84 & 5.86 & 6.45 & 8.07 & 6.36 & 6.41 \\
\hline $\mathrm{Ta}$ & 0.710 & 1.18 & 1.21 & 1.11 & 0.90 & 1.07 & 1.17 & 1.31 & 0.740 & 0.95 & 0.93 \\
\hline Th & 22.7 & 31.1 & 32.3 & 28.3 & 25.8 & 23.1 & 28.5 & 30.4 & 20.2 & 27.5 & 25.5 \\
\hline $\mathrm{U}$ & 5.81 & 9.14 & 8.70 & 8.50 & 7.23 & 7.42 & 8.56 & 7.48 & 4.70 & 7.57 & 7.35 \\
\hline
\end{tabular}

Table S3-continued

\begin{tabular}{|c|c|c|c|c|c|c|c|c|c|c|}
\hline 'Sample & $\begin{array}{c}06 \text { YJL- } \\
20\end{array}$ & $\begin{array}{c}\text { 06YJL- } \\
21\end{array}$ & $\begin{array}{c}\text { 06YJL- } \\
22\end{array}$ & $\begin{array}{c}06 \text { YJL- } \\
23\end{array}$ & $\begin{array}{c}\text { 06YJL- } \\
24\end{array}$ & $\begin{array}{c}06 \mathrm{ZZ} \\
-12\end{array}$ & $\begin{array}{c}06 \mathrm{ZZ} \\
-13\end{array}$ & $\begin{array}{c}06 \mathrm{ZZ} \\
-14\end{array}$ & $\begin{array}{c}06 \mathrm{ZZ} \\
-15\end{array}$ \\
\hline Location & \multicolumn{7}{|c|}{ Yuejinla } \\
\hline $\mathrm{SiO}_{2}$ & 61.09 & 62.86 & 61.31 & 62.56 & 61.28 & 64.1 & 62.68 & 57.25 & 63.89 \\
\hline $\mathrm{TiO}_{2}$ & 0.77 & 0.76 & 0.81 & 0.81 & 0.92 & 0.6 & 0.84 & 0.89 & 0.60 \\
\hline $\mathrm{Al}_{2} \mathrm{O}_{3}$ & 14.93 & 15.85 & 15.67 & 15.48 & 14.82 & 16.1 & 16.06 & 16.47 & 15.31 \\
\hline $\mathrm{Fe}_{2} \mathrm{O}_{3}{ }^{\mathrm{T}}$ & 5.04 & 4.91 & 5.44 & 5.24 & 5.29 & 4.5 & 5.26 & 6.26 & 4.29 \\
\hline $\mathrm{MnO}$ & 0.07 & 0.06 & 0.08 & 0.07 & 0.07 & 0.1 & 0.07 & 0.11 & 0.07 \\
\hline $\mathrm{MgO}$ & 4.42 & 3.29 & 3.68 & 4.02 & 4.31 & 2.5 & 3.35 & 4.99 & 3.51 \\
\hline $\mathrm{CaO}$ & 4.65 & 4.28 & 4.39 & 4.43 & 5.01 & 4.8 & 4.42 & 6.39 & 4.21 \\
\hline $\mathrm{Na}_{2} \mathrm{O}$ & 2.40 & 3.41 & 3.19 & 3.22 & 2.92 & 4.2 & 3.50 & 3.35 & 3.58 \\
\hline $\mathrm{K}_{2} \mathrm{O}$ & 4.48 & 4.01 & 4.33 & 4.41 & 4.37 & 2.7 & 3.30 & 3.35 & 3.51 \\
\hline $\mathrm{P}_{2} \mathrm{O}_{5}$ & 0.09 & 0.10 & 0.25 & 0.15 & 0.82 & 0.1 & 0.06 & 0.09 & 0.16 \\
\hline
\end{tabular}




\begin{tabular}{|c|c|c|c|c|c|c|c|c|c|}
\hline LOI & 1.92 & 0.58 & 0.28 & 0.18 & 0.43 & 1.2 & 0.90 & 1.07 & 0.81 \\
\hline total & 99.85 & 100.11 & 99.43 & 100.57 & 100.24 & 99.6 & 100.43 & 100.22 & 99.93 \\
\hline $\mathrm{Mg \#}$ & 63.7 & 57.3 & 57.5 & 60.5 & 62.0 & 52.6 & 56.0 & 61.5 & 62.1 \\
\hline $\mathrm{Cr}$ & 183 & 152 & 144 & 147 & 245 & 92.2 & 111 & 314 & 168 \\
\hline $\mathrm{Ni}$ & 91.6 & 81.8 & 69.7 & 74.0 & 126 & 67.3 & 64.1 & 119 & 114 \\
\hline $\mathrm{Rb}$ & 164 & 165 & 162 & 159 & 156 & 70.1 & 102 & 90.3 & 122 \\
\hline $\mathrm{Sr}$ & 1609 & 1704 & 1723 & 1703 & 2147 & 1225 & 1051 & 1250 & 1495 \\
\hline Y & 11.6 & 9.94 & 12.0 & 11.6 & 12.3 & 9.2 & 8.38 & 8.73 & 9.78 \\
\hline $\mathrm{Zr}$ & 269 & 305 & 297 & 296 & 364 & 158 & 267 & 229 & 205 \\
\hline $\mathrm{Nb}$ & 12.7 & 14.1 & 15.1 & 14.7 & 13.8 & 5.6 & 10.3 & 9.06 & 7.53 \\
\hline $\mathrm{Ba}$ & 2039 & 2219 & 2179 & 2141 & 2731 & 1415 & 1619 & 1403 & 1705 \\
\hline $\mathrm{La}$ & 70.5 & 58.6 & 77.2 & 72.9 & 45.4 & 22.7 & 43.9 & 22.5 & 52.4 \\
\hline $\mathrm{Ce}$ & 123 & 100.2 & 138 & 124 & 86.3 & 39.6 & 73.3 & 40.7 & 94.3 \\
\hline $\operatorname{Pr}$ & 14.0 & 11.1 & 15.1 & 13.8 & 10.4 & 4.6 & 7.68 & 4.82 & 10.2 \\
\hline $\mathrm{Nd}$ & 47.2 & 38.3 & 52.3 & 46.3 & 40.5 & 17.3 & 25.4 & 18.4 & 35.5 \\
\hline $\mathrm{Sm}$ & 6.53 & 5.40 & 7.02 & 6.49 & 6.76 & 3.1 & 3.69 & 3.22 & 5.14 \\
\hline $\mathrm{Eu}$ & 1.59 & 1.74 & 1.86 & 1.74 & 2.14 & 0.9 & 1.33 & 1.37 & 1.39 \\
\hline $\mathrm{Gd}$ & 3.50 & 3.02 & 3.92 & 3.61 & 3.65 & 2.2 & 2.08 & 2.30 & 2.70 \\
\hline $\mathrm{Tb}$ & 0.520 & 0.441 & 0.558 & 0.507 & 0.561 & 0.4 & 0.34 & 0.33 & 0.40 \\
\hline Dy & 2.47 & 2.11 & 2.59 & 2.40 & 2.68 & 1.9 & 1.69 & 1.86 & 2.03 \\
\hline Но & 0.430 & 0.387 & 0.446 & 0.412 & 0.469 & 0.4 & 0.31 & 0.35 & 0.38 \\
\hline $\mathrm{Er}$ & 1.10 & 0.958 & 1.16 & 1.08 & 1.20 & 1.0 & 0.84 & 0.93 & 0.99 \\
\hline $\mathrm{Tm}$ & 0.15 & 0.129 & 0.149 & 0.144 & 0.163 & 0.2 & 0.12 & 0.13 & 0.14 \\
\hline $\mathrm{Yb}$ & 0.93 & 0.82 & 0.95 & 0.900 & 1.02 & 1.0 & 0.76 & 0.84 & 0.85 \\
\hline $\mathrm{Lu}$ & 0.150 & 0.140 & 0.149 & 0.144 & 0.163 & 0.2 & 0.12 & 0.14 & 0.14 \\
\hline $\mathrm{Hf}$ & 6.31 & 6.76 & 7.08 & 6.77 & 8.66 & 4.2 & 5.85 & 5.44 & 4.99 \\
\hline $\mathrm{Ta}$ & 0.90 & 1.022 & 1.11 & 1.07 & 0.867 & 0.4 & 0.65 & 0.62 & 0.50 \\
\hline $\mathrm{Th}$ & 26.7 & 27.1 & 28.1 & 26.1 & 21.3 & 9.1 & 19.8 & 12.3 & 18.6 \\
\hline $\mathrm{U}$ & 7.27 & 8.11 & 8.32 & 7.90 & 5.29 & 2.9 & 4.53 & 3.81 & 4.92 \\
\hline
\end{tabular}

Note: Loss on Ignition; $\mathrm{Fe}_{2} \mathrm{O}_{3}{ }^{\mathrm{T}}=$ Total $\mathrm{Fe}_{2} \mathrm{O}_{3}$ content; $\mathrm{Mg} \#=$ molar $100 \times \mathrm{Mg}^{2+} /\left(\mathrm{Mg}^{2+}+\mathrm{Fe}^{2+}\right)$. Samples 06YJL-01 06YJL-20 are provided by Dr. Qiang Wang from Guangzhou institute of Geochemistry, Chinese Academy of Sciences. 
Table S4-Bulk-rock Sr-Nd isotope data for EARs in this study

\begin{tabular}{|c|c|c|c|c|c|c|c|c|c|}
\hline Sample & Rock Type & ${ }^{87} \mathrm{Rb} /{ }^{86} \mathrm{Sr}$ & ${ }^{87} \mathrm{Sr} /{ }^{86} \mathrm{Sr}$ & $\pm 2 \sigma$ & $\left({ }^{87} \mathrm{Sr} /{ }^{86} \mathrm{Sr}\right)_{\mathrm{i}}$ & ${ }^{143} \mathrm{Nd} /{ }^{144} \mathrm{Nd}$ & $\pm 2 \sigma$ & $\left({ }^{143} \mathrm{Nd} /{ }^{144} \mathrm{Nd}\right)_{\mathrm{i}}$ & ${ }^{\varepsilon_{\mathrm{Nd}}(\mathrm{t})}$ \\
\hline 06 DYH-19 & Dacite & 0.0900 & 0.707129 & 0.000012 & 0.7071 & 0.5123696 & 0.000007 & 0.512346 & -4.69 \\
\hline 06 ZZ-12 & Dacite & 0.1102 & 0.707732 & 0.000010 & 0.7077 & 0.5123013 & 0.000007 & 0.512272 & -6.13 \\
\hline 06 TPH-10 & Andesite & 0.0883 & 0.707757 & 0.000012 & 0.7077 & 0.5123038 & 0.000009 & 0.512281 & -5.97 \\
\hline 06 YJL-01 & Dacite & 0.0919 & 0.707167 & 0.000013 & 0.7071 & 0.5123792 & 0.000006 & 0.512355 & -4.51 \\
\hline 06 YJL-04 & Dacite & 0.0879 & 0.707078 & 0.000015 & 0.7070 & 0.5123918 & 0.000008 & 0.512369 & -4.25 \\
\hline 06 YJL-05 & Andesite & 0.0864 & 0.707268 & 0.000015 & 0.7072 & 0.5123652 & 0.000004 & 0.512343 & -4.76 \\
\hline 06YJL-13 & Andesite & 0.0906 & 0.707855 & 0.000012 & 0.7078 & 0.5123024 & 0.000004 & 0.512279 & -6.01 \\
\hline LQS-01 & Andesite & 0.0860 & 0.707550 & 0.000016 & 0.7075 & 0.5124802 & 0.000006 & 0.512458 & -2.51 \\
\hline LQS-11 & Rhyolite & 0.0913 & 0.707769 & 0.000013 & 0.7077 & 0.512454 & 0.000009 & 0.512430 & -3.05 \\
\hline LQS-10 & Rhyolite & 0.0868 & 0.707516 & 0.000011 & 0.7075 & 0.5124627 & 0.000006 & 0.512440 & -2.86 \\
\hline LQS-16 & Dacite & 0.1159 & 0.706421 & 0.000014 & 0.7064 & 0.512459 & 0.000011 & 0.512429 & -3.08 \\
\hline
\end{tabular}

Note: ${ }^{87} \mathrm{Rb} /{ }^{86} \mathrm{Sr}$ and ${ }^{147} \mathrm{Sm} /{ }^{144} \mathrm{Nd}$ during inverse computation of initial isotope values were calculated using $\mathrm{Rb}, \mathrm{Sr}, \mathrm{Sm}$, and $\mathrm{Nd}$ contents (Table S3) measured by inductively coupled plasma-mass spectrometry (ICP-MS). 
Table S5-In-situ major elements for cpx measured by EPMA

\begin{tabular}{|c|c|c|c|c|c|c|c|c|c|c|c|c|}
\hline Sample & \multicolumn{10}{|c|}{$\mathrm{YJL}-24\left(\mathrm{SiO}_{2}=64.56 \mathrm{wt} \%, \mathrm{Mg} \#=61.3\right)$} \\
\hline $\mathrm{NO}$ & 0 & 10 & 20 & 30 & 40 & 50 & 60 & 70 & 80 & 90 & 100 \\
\hline $\mathrm{SiO}_{2}$ & 53.65 & 53.60 & 53.79 & 53.35 & 53.77 & 53.15 & 53.93 & 53.19 & 51.99 & 51.89 & 51.53 \\
\hline $\mathrm{TiO}_{2}$ & 0.32 & 0.31 & 0.32 & 0.37 & 0.34 & 0.46 & 0.25 & 0.65 & 0.75 & 0.79 & 0.65 \\
\hline $\mathrm{Al}_{2} \mathrm{O}_{3}$ & 1.76 & 1.94 & 1.85 & 2.27 & 1.79 & 2.68 & 1.59 & 2.38 & 2.23 & 2.34 & 2.69 \\
\hline $\mathrm{FeO}$ & 4.82 & 4.75 & 4.75 & 5.35 & 5.08 & 5.13 & 6.48 & 10.00 & 10.01 & 10.10 & 10.12 \\
\hline $\mathrm{MnO}$ & 0.11 & 0.12 & 0.07 & 0.14 & 0.15 & 0.14 & 0.19 & 0.18 & 0.24 & 0.23 & 0.22 \\
\hline $\mathrm{MgO}$ & 17.37 & 17.34 & 17.35 & 17.48 & 17.55 & 17.11 & 16.78 & 14.52 & 14.74 & 14.71 & 14.48 \\
\hline $\mathrm{CaO}$ & 20.61 & 20.29 & 20.62 & 19.71 & 20.13 & 20.27 & 19.27 & 18.12 & 18.70 & 18.48 & 18.44 \\
\hline $\mathrm{Na} 2 \mathrm{O}$ & 0.48 & 0.50 & 0.47 & 0.49 & 0.52 & 0.34 & 0.47 & 0.74 & 0.67 & 0.68 & 0.77 \\
\hline $\mathrm{K} 2 \mathrm{O}$ & 0.01 & 0.00 & 0.01 & 0.02 & 0.01 & 0.03 & 0.02 & 0.01 & 0.01 & 0.01 & 0.00 \\
\hline $\mathrm{Cr} \mathrm{O}_{3}$ & 0.30 & 0.47 & 0.51 & 0.37 & 0.37 & 0.31 & 0.40 & 0.08 & 0.08 & 0.06 & 0.10 \\
\hline $\mathrm{NiO}$ & 0.04 & 0.06 & 0.09 & 0.08 & 0.00 & 0.10 & 0.07 & 0.06 & 0.06 & 0.02 & 0.06 \\
\hline $\mathrm{Total}$ & 99.46 & 99.37 & 99.84 & 99.63 & 99.72 & 99.69 & 99.47 & 99.93 & 99.47 & 99.31 & 99.06 \\
\hline $\mathrm{Mg} \#$ & 86.64 & 86.78 & 86.80 & 85.46 & 86.15 & 85.73 & 82.34 & 72.33 & 72.61 & 72.39 & 72.03 \\
\hline $\begin{array}{c}\mathrm{Melt} \\
\mathrm{Mg} \#(\mathrm{a})\end{array}$ & 63.85 & 64.15 & 64.16 & 61.56 & 62.87 & 62.05 & 55.93 & 41.58 & 41.92 & 41.65 & 41.22 \\
\hline
\end{tabular}

Table S5-continued

\begin{tabular}{|c|c|c|c|c|c|c|c|c|c|c|c|}
\hline Sample & \multicolumn{10}{|c|}{$\mathrm{YJL}-04\left(\mathrm{SiO}_{2}=61.30 \mathrm{wt} \%\right.$ Mg\# $\left.=61.9\right)$} \\
\hline $\mathrm{NO}$ & 0 & 10 & 20 & 30 & 40 & 50 & 60 & 70 & 80 & 90 & 100 \\
\hline $\mathrm{SiO}_{2}$ & 53.66 & 53.72 & 53.33 & 52.72 & 51.86 & 52.02 & 51.56 & 51.43 & 52.28 & 52.86 & 53.27 \\
\hline $\mathrm{TiO}_{2}$ & 0.27 & 0.51 & 0.37 & 0.70 & 0.66 & 0.77 & 0.75 & 0.67 & 0.71 & 0.71 & 0.71 \\
\hline $\mathrm{Al}_{2} \mathrm{O}_{3}$ & 1.71 & 2.56 & 2.42 & 2.03 & 2.25 & 2.13 & 2.51 & 2.33 & 2.75 & 2.42 & 2.77 \\
\hline $\mathrm{FeO}$ & 4.30 & 5.52 & 5.00 & 7.14 & 7.01 & 7.26 & 10.07 & 9.53 & 9.60 & 9.62 & 8.98 \\
\hline $\mathrm{MnO}$ & 0.13 & 0.12 & 0.13 & 0.17 & 0.16 & 0.18 & 0.24 & 0.18 & 0.15 & 0.20 & 0.22 \\
\hline $\mathrm{MgO}$ & 17.83 & 16.79 & 17.45 & 15.73 & 16.02 & 15.99 & 14.39 & 15.05 & 14.29 & 14.39 & 14.85 \\
\hline $\mathrm{CaO}$ & 19.83 & 19.82 & 19.54 & 20.54 & 20.46 & 20.39 & 18.60 & 19.28 & 18.93 & 18.89 & 18.25 \\
\hline $\mathrm{Na} \mathrm{O}_{2}$ & 0.52 & 0.45 & 0.41 & 0.45 & 0.47 & 0.43 & 0.76 & 0.68 & 0.69 & 0.76 & 0.68 \\
\hline $\mathrm{K} 2 \mathrm{O}$ & 0.00 & 0.03 & 0.00 & 0.01 & 0.03 & 0.00 & 0.00 & 0.02 & 0.00 & 0.00 & 0.00 \\
\hline $\mathrm{Cr} 2 \mathrm{O}_{3}$ & 0.61 & 0.52 & 0.55 & 0.09 & 0.36 & 0.07 & 0.09 & 0.05 & 0.06 & 0.01 & 0.12 \\
\hline $\mathrm{NiO}$ & 0.01 & 0.08 & 0.07 & 0.02 & 0.10 & 0.04 & 0.01 & 0.03 & 0.01 & 0.02 & 0.00 \\
\hline $\mathrm{Total}$ & 98.89 & 100.1 & 99.29 & 99.59 & 99.38 & 99.26 & 98.98 & 99.29 & 99.48 & 99.87 & 99.87 \\
\hline $\mathrm{Mg} \#$ & 88.18 & 84.56 & 86.27 & 79.86 & 80.44 & 79.86 & 72.01 & 73.98 & 72.82 & 72.92 & 74.85 \\
\hline $\begin{array}{l}\mathrm{Melt} \\
\mathrm{Mg} \#\end{array}$ & 64.45 & 63.41 & 64.30 & 59.04 & 60.72 & 60.44 & 52.12 & 42.45 & 41.17 & 41.12 & 41.84 \\
\hline
\end{tabular}

(a) The melt $\mathrm{Mg \#}$ values are inversely calculated by using the Fe-Mg exchange coefficient $(0.275$ $\pm 0.067)$ between clinopyroxene and the equilibrated melt $\left[\mathrm{K}_{\mathrm{D}}(\mathrm{Fe}-\mathrm{Mg})^{\mathrm{Cpx}-\mathrm{melt}}=\right.$ $(\mathrm{Fe} / \mathrm{Mg})^{\mathrm{Cpx}} /(\mathrm{Fe} / \mathrm{Mg})^{\text {melt }}$ where $\mathrm{Fe}$ and $\mathrm{Mg}$ are in molar fraction] of Putirka (1999) and Putirka et al. (2003).

\section{References}

Putirka, K.D., 1999. Clinopyroxene + liquid equilibria to $100 \mathrm{kbar}$ and $2450 \mathrm{~K}$. Contributions to Mineralogy and Petrology 135, 151-163.

Putirka, K.D., Mikaelian, H., Ryerson, F., Shaw, H., 2003. New clinopyroxene-liquid thermobarometers for mafic, evolved, and volatile-bearing lava compositions, with applications to lavas from Tibet and the Snake River Plain, Idaho. American Mineralogist 88, 1542-1554. 
Table S6-In-situ major and trace elements of cpx measured by LA-ICP-MS

\begin{tabular}{|c|c|c|c|c|c|c|c|c|c|c|c|c|c|}
\hline No. & \multicolumn{5}{|c|}{ 06YJL-04 (circle two) } & \multicolumn{8}{|c|}{ 06YJL-24 (circle one) } \\
\hline Type & Rim & Core & Core & Rim & Rim & Rim & Rim & Rim & Core & Core & Core & Core & Core \\
\hline $\mathrm{SiO}_{2}$ & 52.17 & 51.14 & 51.20 & 51.19 & 51.00 & 52.19 & 51.79 & 50.96 & 50.74 & 51.10 & 50.07 & 50.6 & 50.5 \\
\hline $\mathrm{TiO}_{2}$ & 0.41 & 0.53 & 0.71 & 0.57 & 0.66 & 0.28 & 0.33 & 0.50 & 0.62 & 0.56 & 0.74 & 0.66 & 0.73 \\
\hline $\mathrm{Al}_{2} \mathrm{O}_{3}$ & 2.30 & 2.68 & 3.38 & 3.45 & 2.60 & 1.53 & 2.07 & 2.54 & 2.48 & 2.52 & 2.45 & 2.41 & 2.73 \\
\hline $\mathrm{FeO}$ & 6.70 & 8.73 & 9.43 & 7.48 & 7.37 & 5.76 & 5.31 & 7.16 & 8.85 & 7.87 & 9.68 & 9.91 & 9.83 \\
\hline $\mathrm{MgO}$ & 16.95 & 15.72 & 14.04 & 15.61 & 16.27 & 18.28 & 17.82 & 16.67 & 15.88 & 16.32 & 15.47 & 15.4 & 15.1 \\
\hline $\mathrm{MnO}$ & 0.16 & 0.21 & 0.22 & 0.17 & 0.17 & 0.15 & 0.13 & 0.17 & 0.21 & 0.20 & 0.23 & 0.24 & 0.25 \\
\hline $\mathrm{CaO}$ & 19.85 & 19.72 & 19.26 & 20.06 & 20.82 & 20.72 & 21.26 & 20.71 & 19.88 & 20.17 & 20.11 & 19.5 & 19.5 \\
\hline $\mathrm{Na}_{2} \mathrm{O}$ & 0.59 & 0.64 & 0.76 & 0.73 & 0.47 & 0.36 & 0.42 & 0.56 & 0.63 & 0.59 & 0.66 & 0.71 & 0.73 \\
\hline $\mathrm{K}_{2} \mathrm{O}$ & 0.03 & 0.04 & 0.41 & 0.08 & 0.07 & 0.01 & 0.00 & 0.00 & 0.00 & 0.00 & 0.00 & 0.00 & 0.01 \\
\hline $\mathrm{P}_{2} \mathrm{O}_{5}$ & 0.01 & 0.03 & 0.06 & 0.01 & 0.00 & 0.03 & 0.01 & 0.00 & 0.04 & 0.01 & 0.02 & 0.00 & 0.01 \\
\hline $\mathrm{Mg} \#$ & 82.0 & 76.4 & 72.8 & 78.9 & 79.9 & 85.1 & 85.8 & 80.7 & 76.4 & 78.8 & 74.2 & 73.7 & 73.4 \\
\hline Melt & 53.97 & 45.5 & 40.8 & 49.2 & 50.57 & 59.53 & 60.7 & 51.9 & 45.4 & 49.0 & 42.6 & 53.97 & 45.5 \\
\hline $\mathrm{Li}$ & 25.86 & 33.59 & 38.27 & 41.30 & 29.03 & 52.07 & 34.99 & 46.81 & 38.20 & 33.13 & 44.53 & 20.6 & 48.1 \\
\hline $\mathrm{Sc}$ & 39.53 & 55.84 & 55.51 & 46.25 & 50.76 & 36.00 & 34.02 & 46.83 & 56.44 & 50.28 & 51.64 & 54.6 & 59.3 \\
\hline $\mathrm{V}$ & 145 & 206 & 210 & 161 & 154 & 90 & 107 & 166 & 188 & 180 & 191 & 199 & 226 \\
\hline $\mathrm{Cr}$ & 3149 & 578 & 324 & 1122 & 1169 & 2271 & 3279 & 1979 & 1442 & 1681 & 639 & 850 & 887 \\
\hline Co & 41.38 & 46.39 & 48.45 & 45.18 & 41.52 & 40.04 & 40.08 & 42.49 & 46.58 & 45.87 & 50.5 & 49.7 & 47.7 \\
\hline $\mathrm{Ni}$ & 614 & 423 & 271 & 462 & 461 & 775 & 771 & 609 & 463 & 510 & 330 & 314 & 362 \\
\hline $\mathrm{Cu}$ & 2.90 & 13.93 & 14.92 & 13.59 & 11.43 & 36.15 & 3.60 & 9.57 & 7.45 & 0.00 & 0.89 & 5.50 & 3.96 \\
\hline $\mathrm{Zn}$ & 82.4 & 113.1 & 129.4 & 78.3 & 64.0 & 52.4 & 50.6 & 71.7 & 120 & 101.3 & 133 & 156 & 142 \\
\hline $\mathrm{Rb}$ & 1.05 & 0.86 & 13.74 & 1.09 & 2.85 & 0.02 & 0.00 & 0.00 & 0.12 & 0.00 & 0.00 & 0.03 & 0.24 \\
\hline $\mathrm{Sr}$ & 268 & 137 & 183 & 156 & 187 & 187 & 217 & 177 & 149 & 159 & 148 & 100 & 90 \\
\hline $\mathrm{Y}$ & 20.2 & 31.7 & 38.4 & 27.0 & 23.1 & 8.5 & 8.9 & 24.1 & 36.3 & 32.5 & 39.8 & 45.0 & 48.6 \\
\hline $\mathrm{Zr}$ & 74.8 & 107.5 & 128.6 & 84.1 & 58.4 & 12.7 & 19.6 & 70.7 & 105.8 & 91.8 & 110.8 & 121 & 157 \\
\hline $\mathrm{Nb}$ & 0.13 & 0.37 & 1.29 & 0.21 & 0.12 & 0.03 & 0.03 & 0.02 & 0.13 & 0.15 & 0.16 & 0.20 & 0.20 \\
\hline $\mathrm{Ba}$ & 13.4 & 5.4 & 180.1 & 87.9 & 18.9 & 0.3 & 0.2 & 0.6 & 0.10 & 0.20 & 0.20 & 0.0 & 8.0 \\
\hline $\mathrm{La}$ & 12.7 & 21.7 & 32.1 & 20.3 & 18.5 & 5.2 & 6.0 & 16.5 & 27.8 & 22.7 & 32.7 & 33.5 & 34.1 \\
\hline $\mathrm{Ce}$ & 50.2 & 86.3 & 115 & 72.7 & 64.9 & 21.1 & 24.2 & 65.3 & 102 & 82.9 & 123 & 128 & 135 \\
\hline $\operatorname{Pr}$ & 8.6 & 14.6 & 19.0 & 13.3 & 11.9 & 3.6 & 4.1 & 11.2 & 18.0 & 13.8 & 20.1 & 21.7 & 23.0 \\
\hline $\mathrm{Nd}$ & 54.3 & 65.6 & 82.7 & 60.2 & 55.1 & 20.8 & 22.5 & 52.6 & 83.8 & 67.4 & 93.4 & 105 & 108 \\
\hline $\mathrm{Sm}$ & 8.84 & 16.28 & 18.02 & 12.70 & 12.53 & 5.56 & 5.89 & 11.7 & 17.39 & 15.87 & 21.04 & 21.7 & 22.6 \\
\hline
\end{tabular}




\begin{tabular}{|c|c|c|c|c|c|c|c|c|c|c|c|c|c|}
\hline $\mathrm{Eu}$ & 1.87 & 2.59 & 3.94 & 3.01 & 3.71 & 1.62 & 1.57 & 2.66 & 3.36 & 2.93 & 3.95 & 4.06 & 3.89 \\
\hline $\mathrm{Gd}$ & 5.59 & 10.24 & 12.11 & 7.52 & 8.29 & 3.52 & 3.66 & 7.23 & 11.72 & 10.84 & 14.12 & 14.1 & 14.1 \\
\hline $\mathrm{Tb}$ & 0.93 & 1.52 & 1.63 & 1.22 & 1.19 & 0.43 & 0.38 & 1.21 & 1.87 & 1.45 & 1.75 & 1.96 & 2.14 \\
\hline Dy & 5.39 & 6.67 & 8.73 & 5.77 & 5.60 & 2.29 & 2.14 & 5.37 & 8.06 & 7.40 & 8.29 & 10.1 & 10.5 \\
\hline Но & 0.78 & 1.19 & 1.52 & 0.97 & 0.90 & 0.34 & 0.35 & 0.87 & 1.40 & 1.25 & 1.32 & 1.63 & 1.77 \\
\hline Er & 2.07 & 3.88 & 3.35 & 2.98 & 2.29 & 1.20 & 0.99 & 2.40 & 3.68 & 3.19 & 3.82 & 4.64 & 5.10 \\
\hline $\mathrm{Tm}$ & 0.28 & 0.42 & 0.48 & 0.33 & 0.25 & 0.10 & 0.09 & 0.28 & 0.43 & 0.41 & 0.51 & 0.56 & 0.63 \\
\hline $\mathrm{Yb}$ & 1.78 & 2.45 & 3.44 & 2.17 & 2.11 & 0.63 & 0.66 & 1.90 & 2.98 & 2.37 & 3.19 & 3.98 & 3.49 \\
\hline $\mathrm{Lu}$ & 0.20 & 0.30 & 0.38 & 0.28 & 0.21 & 0.07 & 0.07 & 0.28 & 0.45 & 0.35 & 0.45 & 0.51 & 0.50 \\
\hline $\mathrm{Hf}$ & 2.73 & 3.38 & 4.48 & 2.58 & 2.29 & 0.72 & 0.72 & 2.82 & 3.71 & 3.39 & 3.46 & 3.86 & 5.52 \\
\hline $\mathrm{Ta}$ & 0.02 & 0.01 & 0.09 & 0.01 & 0.04 & 0.01 & 0.00 & 0.02 & 0.02 & 0.02 & 0.01 & 0.02 & 0.02 \\
\hline $\mathrm{Pb}$ & 1.15 & 1.75 & 6.10 & 2.17 & 1.45 & 0.43 & 0.43 & 0.75 & 1.27 & 0.96 & 1.47 & 1.56 & 1.58 \\
\hline Th & 0.38 & 0.46 & 3.05 & 0.70 & 0.66 & 0.05 & 0.10 & 0.21 & 0.42 & 0.30 & 0.50 & 0.46 & 0.50 \\
\hline $\mathrm{U}$ & 0.05 & 0.10 & 0.76 & 0.09 & 0.16 & 0.01 & 0.02 & 0.04 & 0.06 & 0.04 & 0.07 & 0.06 & 0.09 \\
\hline $\begin{array}{l}\text { Melt } \\
\text { Mg\# }\end{array}$ & 53.97 & 45.5 & 40.8 & 49.2 & 50.57 & 59.53 & 60.7 & 51.9 & 45.4 & 49.0 & 42.6 & 53.97 & 45.5 \\
\hline $\begin{array}{c}\text { Melt } \\
\text { Sr }\end{array}$ & 1340 & 685 & 915 & 780 & 935 & 935 & 1085 & 885 & 745 & 795 & 740 & 500 & 450 \\
\hline $\begin{array}{c}\text { Melt } \\
\text { Y }\end{array}$ & 1340 & 685 & 915 & 780 & 935 & 935 & 1085 & 885 & 745 & 795 & 740 & 500 & 450 \\
\hline $\begin{array}{l}\text { Melt } \\
\mathrm{Sr} / \mathrm{Y}\end{array}$ & 60 & 20 & 22 & 26 & 37 & 100 & 111 & 33 & 19 & 22 & 17 & 10 & 8 \\
\hline
\end{tabular}

Table S6-continued

\begin{tabular}{|c|c|c|c|c|c|c|c|c|c|c|c|c|c|}
\hline No & \multicolumn{7}{|c|}{ YJL-24 (circle two) } & \multicolumn{5}{c|}{ YJL-04 (circle one) } \\
\hline $\mathrm{Type}$ & $\mathrm{Rim}$ & $\mathrm{Rim}$ & $\mathrm{Rim}$ & Rim & Core & Core & Core & Rim & Rim & Rim & Rim & Core & Core \\
\hline $\mathrm{SiO}_{2}$ & 52.06 & 52.28 & 52.34 & 52.83 & 51.30 & 51.88 & 50.71 & 52.61 & 52.18 & 52.29 & 51.41 & 50.13 & 50.64 \\
\hline $\mathrm{SiO}_{2}$ & 52.06 & 52.28 & 52.34 & 52.83 & 51.30 & 51.88 & 50.71 & 52.61 & 52.18 & 52.29 & 51.41 & 50.13 & 50.64 \\
\hline $\mathrm{TiO}_{2}$ & 0.36 & 0.39 & 0.42 & 0.36 & 0.67 & 0.69 & 0.70 & 0.30 & 0.55 & 0.52 & 0.68 & 0.66 & 0.71 \\
\hline $\mathrm{Al} \mathrm{O}_{3}$ & 1.79 & 2.64 & 3.28 & 2.18 & 3.52 & 2.51 & 2.49 & 1.76 & 2.76 & 2.60 & 3.73 & 3.51 & 4.00 \\
\hline $\mathrm{FeO}$ & 5.44 & 4.73 & 4.81 & 4.82 & 7.58 & 8.82 & 8.76 & 5.04 & 5.85 & 6.00 & 5.99 & 8.26 & 8.17 \\
\hline $\mathrm{MgO}$ & 18.31 & 17.54 & 16.90 & 17.87 & 15.03 & 14.79 & 14.86 & 18.70 & 16.51 & 16.56 & 16.00 & 14.74 & 14.01 \\
\hline $\mathrm{MnO}$ & 0.15 & 0.13 & 0.13 & 0.14 & 0.18 & 0.24 & 0.24 & 0.14 & 0.16 & 0.16 & 0.14 & 0.19 & 0.18 \\
\hline $\mathrm{CaO}$ & 20.85 & 20.86 & 20.53 & 20.54 & 19.70 & 19.76 & 20.61 & 20.09 & 21.04 & 20.90 & 20.87 & 21.29 & 20.62 \\
\hline $\mathrm{Na} 2 \mathrm{O}$ & 0.38 & 0.53 & 0.63 & 0.46 & 0.69 & 0.70 & 0.70 & 0.44 & 0.47 & 0.48 & 0.54 & 0.63 & 0.74 \\
\hline $\mathrm{K} 2 \mathrm{O}$ & 0.00 & 0.00 & 0.11 & 0.01 & 0.72 & 0.03 & 0.00 & 0.04 & 0.02 & 0.04 & 0.08 & 0.03 & 0.28 \\
\hline
\end{tabular}




\begin{tabular}{|c|c|c|c|c|c|c|c|c|c|c|c|c|c|}
\hline $\mathrm{P}_{2} \mathrm{O}_{5}$ & 0.02 & 0.04 & 0.04 & 0.02 & 0.04 & 0.02 & 0.30 & 0.03 & 0.02 & 0.02 & 0.07 & 0.02 & 0.04 \\
\hline Mg\# & 85.84 & 86.98 & 86.34 & 86.98 & 78.12 & 75.12 & 75.34 & 86.97 & 83.55 & 83.24 & 82.78 & 76.25 & 75.52 \\
\hline $\mathrm{Li}$ & 33.15 & 2.53 & 65.94 & 34.17 & 47.82 & 43.30 & 45.62 & 10.66 & 14.99 & 39.09 & 26.92 & 0.00 & 36.93 \\
\hline $\mathrm{Sc}$ & 36.66 & 37.79 & 38.24 & 37.27 & 44.95 & 58.55 & 56.49 & 31.11 & 41.12 & 39.52 & 43.81 & 46.92 & 40.50 \\
\hline $\mathrm{V}$ & 100 & 127 & 141 & 109 & 161 & 217 & 213 & 97 & 153 & 141 & 181 & 169 & 168 \\
\hline $\mathrm{Cr}$ & 2061 & 3752 & 3138 & 3071 & 1038 & 785 & 805 & 3113 & 747 & 669 & 790 & 804 & 895 \\
\hline Co & 42.04 & 36.88 & 34.88 & 39.77 & 40.51 & 52.15 & 49.93 & 40.39 & 44.15 & 43.62 & 40.55 & 43.69 & 42.26 \\
\hline $\mathrm{Ni}$ & 751 & 782 & 797 & 800 & 418 & 379 & 347 & 820 & 594 & 586 & 577 & 337 & 261 \\
\hline $\mathrm{Cu}$ & 6.44 & 4.24 & 2.21 & 8.02 & 15.45 & 22.27 & 7.86 & 158.37 & 26.81 & 8.27 & 5.34 & 5.21 & 0.32 \\
\hline $\mathrm{Zn}$ & 62.2 & 53.5 & 59.9 & 56.8 & 82.3 & 148.0 & 151.4 & 71.5 & 61.7 & 50.2 & 62.7 & 116.3 & 121.5 \\
\hline $\mathrm{Rb}$ & 0.00 & 0.00 & 3.81 & 0.49 & 16.03 & 1.02 & 0.02 & 1.31 & 0.76 & 1.41 & 4.64 & 2.60 & 13.87 \\
\hline $\mathrm{Sr}$ & 181 & 212 & 267 & 194 & 187 & 109 & 108 & 181 & 217 & 219 & 257 & 126 & 141 \\
\hline $\mathrm{Y}$ & 11.2 & 11.8 & 13.4 & 9.6 & 29.2 & 40.2 & 43.7 & 8.9 & 15.6 & 17.0 & 18.2 & 29.4 & 29.6 \\
\hline $\mathrm{Zr}$ & 24.5 & 25.1 & 32.2 & 19.2 & 116.4 & 117.9 & 116.5 & 17.4 & 39.7 & 40.5 & 55.7 & 102.5 & 120.2 \\
\hline $\mathrm{Nb}$ & 0.00 & 0.09 & 0.38 & 0.00 & 1.74 & 0.28 & 0.26 & 0.09 & 0.29 & 0.18 & 0.33 & 0.36 & 1.25 \\
\hline $\mathrm{Ba}$ & 5.6 & 0.3 & 63.5 & 0.8 & 81.3 & 2.6 & 0.6 & 5.7 & 1.2 & 17.0 & 10.7 & 9.8 & 87.6 \\
\hline $\mathrm{La}$ & 6.9 & 6.8 & 9.8 & 5.8 & 27.9 & 26.2 & 38.5 & 4.6 & 9.8 & 11.9 & 13.6 & 16.6 & 19.3 \\
\hline $\mathrm{Ce}$ & 27.5 & 26.4 & 31.5 & 22.9 & 90.6 & 100.8 & 129.4 & 18.7 & 38.2 & 43.3 & 49.8 & 63.7 & 76.7 \\
\hline $\operatorname{Pr}$ & 4.9 & 5.0 & 5.8 & 4.0 & 14.9 & 17.5 & 21.3 & 3.2 & 7.5 & 7.6 & 8.8 & 10.9 & 14.0 \\
\hline $\mathrm{Nd}$ & 23.5 & 25.2 & 29.1 & 21.5 & 65.5 & 81.7 & 91.4 & 17.1 & 32.9 & 40.5 & 46.5 & 54.9 & 71.8 \\
\hline $\mathrm{Sm}$ & 6.41 & 5.28 & 6.26 & 4.91 & 14.38 & 18.46 & 20.98 & 4.94 & 7.61 & 7.93 & 9.92 & 12.09 & 15.94 \\
\hline $\mathrm{Eu}$ & 1.67 & 1.71 & 2.06 & 1.74 & 3.16 & 3.54 & 4.03 & 1.35 & 2.59 & 2.62 & 2.75 & 2.65 & 3.33 \\
\hline $\mathrm{Gd}$ & 4.15 & 2.83 & 4.12 & 3.23 & 9.02 & 11.95 & 13.28 & 2.39 & 5.44 & 5.97 & 6.10 & 11.81 & 15.80 \\
\hline $\mathrm{Tb}$ & 0.60 & 0.54 & 0.58 & 0.39 & 1.35 & 1.62 & 1.81 & 0.36 & 0.65 & 0.83 & 0.88 & 1.44 & 2.16 \\
\hline Dy & 2.83 & 2.69 & 3.21 & 2.89 & 5.86 & 8.48 & 9.09 & 2.26 & 3.37 & 4.34 & 4.56 & 7.12 & 10.51 \\
\hline Но & 0.42 & 0.45 & 0.48 & 0.44 & 1.15 & 1.34 & 1.55 & 0.29 & 0.56 & 0.66 & 0.74 & 1.24 & 1.84 \\
\hline $\mathrm{Er}$ & 1.10 & 1.09 & 1.36 & 0.97 & 2.58 & 4.11 & 4.47 & 0.90 & 1.52 & 1.66 & 1.79 & 3.27 & 4.54 \\
\hline $\mathrm{Tm}$ & 0.11 & 0.16 & 0.13 & 0.11 & 0.43 & 0.44 & 0.52 & 0.11 & 0.14 & 0.18 & 0.17 & 0.54 & 0.82 \\
\hline $\mathrm{Yb}$ & 0.84 & 0.72 & 0.91 & 0.87 & 2.16 & 3.65 & 3.75 & 0.54 & 1.13 & 1.45 & 1.35 & 2.84 & 4.08 \\
\hline $\mathrm{Lu}$ & 0.12 & 0.12 & 0.10 & 0.11 & 0.25 & 0.55 & 0.47 & 0.09 & 0.16 & 0.21 & 0.18 & 0.33 & 0.40 \\
\hline $\mathrm{Hf}$ & 0.72 & 1.12 & 1.29 & 0.89 & 3.83 & 3.67 & 4.12 & 0.67 & 1.55 & 1.56 & 2.29 & 3.24 & 3.18 \\
\hline $\mathrm{Ta}$ & 0.01 & 0.01 & 0.05 & 0.00 & 0.13 & 0.04 & 0.01 & 0.01 & 0.02 & 0.03 & 0.01 & 0.07 & 0.13 \\
\hline $\mathrm{Pb}$ & 0.79 & 1.34 & 2.58 & 1.47 & 6.79 & 1.77 & 2.00 & 1.22 & 0.69 & 1.00 & 1.54 & 1.73 & 4.51 \\
\hline Th & 0.11 & 0.08 & 0.74 & 0.10 & 4.12 & 0.40 & 0.82 & 0.09 & 0.16 & 0.37 & 0.56 & 0.96 & 3.66 \\
\hline
\end{tabular}




\begin{tabular}{|c|c|c|c|c|c|c|c|c|c|c|c|c|c|}
\hline U & 0.02 & 0.02 & 0.20 & 0.01 & 0.91 & 0.06 & 0.14 & 0.03 & 0.04 & 0.07 & 0.15 & 0.15 & 0.84 \\
\hline $\begin{array}{c}\text { Melt } \\
\text { Mg\# }\end{array}$ & 60.93 & 63.22 & 61.95 & 63.21 & 47.89 & 43.73 & 44.01 & 63.23 & 56.67 & 56.12 & 55.31 & 45.27 & 60.93 \\
\hline $\begin{array}{c}\text { Melt } \\
\text { Sr }\end{array}$ & 905 & 1060 & 1335 & 970 & 935 & 545 & 540 & 905 & 1085 & 1095 & 1285 & 630 & 705 \\
\hline $\begin{array}{c}\text { Melt } \\
\text { Y }\end{array}$ & 12.3 & 13.0 & 14.7 & 10.5 & 32.1 & 44.2 & 48.0 & 9.8 & 17.1 & 18.7 & 20.0 & 32.3 & 32.5 \\
\hline $\begin{array}{c}\text { Melt } \\
\text { Sr/Y }\end{array}$ & 74 & 82 & 91 & 92 & 29 & 12 & 11 & 93 & 63 & 59 & 64 & 20 & 22 \\
\hline
\end{tabular}

Table S6-continued

\begin{tabular}{|c|c|c|c|c|c|c|}
\hline No. & $\begin{array}{c}\text { 06YJL- } \\
24\end{array}$ & $\begin{array}{l}\text { 06YJL- } \\
24\end{array}$ & $\begin{array}{c}\text { 06YJL- } \\
04\end{array}$ & $\begin{array}{c}\text { 06YJL- } \\
04\end{array}$ & $\begin{array}{c}\text { 06YJL- } \\
04\end{array}$ & $\begin{array}{c}\text { 06YJL- } \\
04\end{array}$ \\
\hline Type & \multicolumn{6}{|c|}{ Unzoned } \\
\hline $\mathrm{SiO}_{2}$ & 51.75 & 52.45 & 52.53 & 52.68 & 51.74 & 51.24 \\
\hline $\mathrm{TiO}_{2}$ & 0.30 & 0.48 & 0.31 & 0.29 & 0.36 & 0.70 \\
\hline $\mathrm{Al}_{2} \mathrm{O}_{3}$ & 2.28 & 2.43 & 2.45 & 1.79 & 2.48 & 3.05 \\
\hline $\mathrm{FeO}$ & 5.06 & 5.68 & 6.31 & 5.22 & 4.97 & 6.52 \\
\hline $\mathrm{MgO}$ & 18.62 & 16.98 & 18.66 & 18.93 & 17.98 & 16.21 \\
\hline $\mathrm{MnO}$ & 0.14 & 0.15 & 0.16 & 0.15 & 0.13 & 0.16 \\
\hline $\mathrm{CaO}$ & 20.57 & 20.94 & 18.43 & 19.74 & 20.92 & 21.10 \\
\hline $\mathrm{Na}_{2} \mathrm{O}$ & 0.42 & 0.44 & 0.39 & 0.45 & 0.52 & 0.47 \\
\hline $\mathrm{K}_{2} \mathrm{O}$ & 0.00 & 0.01 & 0.02 & 0.01 & 0.02 & 0.03 \\
\hline $\mathrm{P}_{2} \mathrm{O}_{5}$ & 0.02 & 0.01 & 0.02 & 0.03 & 0.02 & 0.03 \\
\hline $\mathrm{Mg} \#$ & 86.89 & 84.33 & 84.19 & 86.71 & 86.70 & 81.73 \\
\hline $\mathrm{Li}$ & 43.11 & 21.49 & 46.15 & 23.14 & 44.75 & 37.63 \\
\hline $\mathrm{Sc}$ & 19.25 & 37.09 & 0.00 & 33.21 & 37.19 & 48.54 \\
\hline V & 154 & 146 & 164 & 109 & 131 & 169 \\
\hline $\mathrm{Cr}$ & 3963 & 622 & 2832 & 2593 & 3331 & 796 \\
\hline Co & 39.05 & 43.30 & 42.21 & 40.19 & 39.02 & 42.89 \\
\hline $\mathrm{Ni}$ & 251 & 628 & 257 & 850 & 808 & 540 \\
\hline $\mathrm{Cu}$ & 3.93 & 4.51 & 5.21 & 4.81 & 9.81 & 7.24 \\
\hline $\mathrm{Zn}$ & 60.1 & 56.1 & 83.0 & 55.3 & 42.3 & 58.0 \\
\hline $\mathrm{Rb}$ & 0.11 & 0.00 & 0.61 & 0.17 & 0.80 & 1.06 \\
\hline $\mathrm{Sr}$ & 148 & 218 & 167 & 172 & 209 & 208 \\
\hline Y & 6.9 & 13.9 & 8.5 & 9.5 & 10.5 & 21.7 \\
\hline $\mathrm{Zr}$ & 11.5 & 33.0 & 16.8 & 18.4 & 21.7 & 62.3 \\
\hline
\end{tabular}




\begin{tabular}{|c|c|c|c|c|c|c|}
\hline $\mathrm{Nb}$ & 0.01 & 0.10 & 0.11 & 0.06 & 0.08 & 0.06 \\
\hline $\mathrm{Ba}$ & 0.1 & 0.3 & 3.2 & 1.4 & 1.3 & 2.6 \\
\hline $\mathrm{La}$ & 4.6 & 8.9 & 7.1 & 4.8 & 7.1 & 14.8 \\
\hline $\mathrm{Ce}$ & 16.2 & 33.4 & 23.1 & 17.9 & 25.8 & 57.4 \\
\hline $\operatorname{Pr}$ & 2.6 & 6.3 & 3.5 & 3.3 & 4.8 & 10.0 \\
\hline $\mathrm{Nd}$ & 14.4 & 27.9 & 15.2 & 15.4 & 21.0 & 51.4 \\
\hline $\mathrm{Sm}$ & 2.78 & 8.03 & 3.46 & 4.76 & 5.60 & 10.77 \\
\hline $\mathrm{Eu}$ & 0.83 & 2.06 & 0.98 & 1.48 & 1.85 & 3.28 \\
\hline $\mathrm{Gd}$ & 2.90 & 5.14 & 3.62 & 2.94 & 3.77 & 7.59 \\
\hline $\mathrm{Tb}$ & 0.44 & 0.68 & 0.40 & 0.42 & 0.46 & 1.04 \\
\hline Dy & 2.68 & 3.19 & 2.57 & 2.27 & 2.78 & 5.21 \\
\hline Ho & 0.35 & 0.51 & 0.42 & 0.31 & 0.37 & 0.92 \\
\hline $\mathrm{Er}$ & 0.81 & 1.32 & 0.91 & 0.86 & 1.03 & 2.32 \\
\hline $\mathrm{Tm}$ & 0.14 & 0.15 & 0.19 & 0.07 & 0.10 & 0.29 \\
\hline $\mathrm{Yb}$ & 0.73 & 0.82 & 0.87 & 0.57 & 0.79 & 1.74 \\
\hline $\mathrm{Lu}$ & 0.09 & 0.12 & 0.10 & 0.10 & 0.10 & 0.22 \\
\hline $\mathrm{Hf}$ & 0.56 & 1.37 & 0.77 & 0.77 & 1.01 & 1.96 \\
\hline $\mathrm{Ta}$ & 0.00 & 0.01 & 0.01 & 0.00 & 0.00 & 0.03 \\
\hline $\mathrm{Pb}$ & 0.45 & 0.65 & 0.67 & 0.89 & 1.23 & 1.07 \\
\hline Th & 0.07 & 0.16 & 0.38 & 0.06 & 0.11 & 0.34 \\
\hline $\mathrm{U}$ & 0.02 & 0.02 & 0.06 & 0.03 & 0.03 & 0.07 \\
\hline $\begin{array}{l}\text { Melt } \\
\text { Mg\# }\end{array}$ & 63.04 & 58.08 & 57.81 & 62.70 & 62.64 & 63.04 \\
\hline $\begin{array}{c}\text { Melt } \\
\mathrm{Sr}\end{array}$ & 740 & 1090 & 835 & 860 & 1045 & 740 \\
\hline $\begin{array}{c}\text { Melt } \\
\text { Y }\end{array}$ & 7.6 & 15.3 & 9.3 & 10.4 & 11.5 & 7.6 \\
\hline $\begin{array}{l}\text { Melt } \\
\mathrm{Sr} / \mathrm{Y}\end{array}$ & 98 & 71 & 89 & 82 & 91 & 98 \\
\hline
\end{tabular}

The melt Mg\# values are inversely calculated by using the same methods of Table S5. The melt Sr and melt $\mathrm{Y}$ refer to inversely calculated concentrations of melt in equilibrium with the cpx. Partition coefficients between andesite/trachyte and cpx ( 0.20 for $\mathrm{Sr}$ and $0.91 \mathrm{for} \mathrm{Y})$ are used because the composition of the EARs (see GERM (Geochemical Earth Reference Model) home page, http://www.earthref.org/). 
Table S7-Published data for EARs

\begin{tabular}{|c|c|c|c|c|c|c|c|c|c|c|c|}
\hline Samples & $\begin{array}{l}13 \mathrm{SW} \\
28-01\end{array}$ & $\begin{array}{l}13 \mathrm{SW} \\
28-02\end{array}$ & $\begin{array}{l}13 \mathrm{SW} \\
29-01\end{array}$ & $\begin{array}{c}13 \mathrm{SW} 29- \\
02\end{array}$ & $\begin{array}{l}13 \mathrm{SW} \\
29-03\end{array}$ & $\begin{array}{c}13 \mathrm{SW} \\
30-1\end{array}$ & $\begin{array}{c}13 S W \\
30-2\end{array}$ & $\begin{array}{c}13 \mathrm{SW} \\
31-1\end{array}$ & $\begin{array}{c}13 \mathrm{SW} \\
31-2\end{array}$ & $\begin{array}{c}13 \mathrm{SW} \\
32-1\end{array}$ & $\begin{array}{c}13 \mathrm{SW} \\
33-1\end{array}$ \\
\hline Ref. & 1 & 1 & 1 & 1 & 1 & 1 & 1 & 1 & 1 & 1 & 1 \\
\hline $\mathrm{SiO}_{2}$ & 69.55 & 69.49 & 68.52 & 68.75 & 69.14 & 69.42 & 69.36 & 69.32 & 69.29 & 70.7 & 70.56 \\
\hline $\mathrm{TiO}_{2}$ & 0.44 & 0.42 & 0.38 & 0.39 & 0.37 & 0.39 & 0.41 & 0.41 & 0.42 & 0.38 & 0.41 \\
\hline $\mathrm{Al}_{2} \mathrm{O}_{3}$ & 14.99 & 14.86 & 14.89 & 14.82 & 15.03 & 15.06 & 15.15 & 14.96 & 14.96 & 14.63 & 14.69 \\
\hline $\mathrm{Fe}_{2} \mathrm{O}_{3}$ & 3.2 & 3.4 & 2.58 & 2.5 & 2.55 & 3.36 & 3.38 & 3.42 & 3.36 & 2.99 & 3.09 \\
\hline $\mathrm{MnO}$ & 0.03 & 0.03 & 0.06 & 0.06 & 0.07 & 0.02 & 0.02 & 0.03 & 0.03 & 0.04 & 0.03 \\
\hline $\mathrm{MgO}$ & 0.4 & 0.41 & 0.46 & 0.5 & 0.54 & 0.43 & 0.36 & 0.36 & 0.36 & 0.37 & 0.42 \\
\hline $\mathrm{CaO}$ & 1.94 & 1.94 & 2.06 & 2.1 & 2.08 & 1.96 & 2.03 & 2.01 & 2.01 & 1.54 & 1.43 \\
\hline $\mathrm{Na}_{2} \mathrm{O}$ & 3.26 & 3.22 & 3.14 & 3.13 & 3.06 & 3.44 & 3.42 & 3.58 & 3.5 & 3.17 & 2.86 \\
\hline $\mathrm{K}_{2} \mathrm{O}$ & 4.25 & 4.16 & 4.21 & 4.2 & 4.18 & 4.11 & 4.06 & 4.19 & 4.1 & 4.23 & 4.21 \\
\hline $\mathrm{P}_{2} \mathrm{O}_{5}$ & 0.15 & 0.15 & 0.13 & 0.13 & 0.12 & 0.12 & 0.12 & 0.13 & 0.13 & 0.13 & 0.14 \\
\hline $\mathrm{Mg} \#$ & 20 & 19 & 26 & 28 & 30 & 20 & 17 & 17 & 17 & 19 & 21 \\
\hline $\mathrm{Cr}$ & 141 & 276 & 268 & 231 & 353 & 106 & 113 & 165 & 153 & 227 & 147 \\
\hline $\mathrm{Ni}$ & 28.7 & 42.5 & 32.9 & 25.1 & 37.7 & 24.8 & 22.1 & 29.7 & 27.9 & 31 & 30.1 \\
\hline $\mathrm{Rb}$ & 191 & 185 & 213 & 202 & 209 & 199 & 176 & 191 & 193 & 215 & 206 \\
\hline $\mathrm{Ba}$ & 1445 & 1320 & 1316 & 1345 & 1338 & 1263 & 1147 & 1231 & 1261 & 1258 & 1271 \\
\hline Th & 27.2 & 27 & 25.1 & 26.1 & 25.5 & 24.9 & 24.4 & 24.8 & 24.5 & 24.2 & 24.9 \\
\hline $\mathrm{Nb}$ & 8.4 & 8.2 & 9.8 & 9.3 & 9.7 & 9 & 8.2 & 9.1 & 8.9 & 10.4 & 10 \\
\hline $\mathrm{Ta}$ & 0.71 & 0.68 & 0.9 & 0.85 & 0.91 & 0.86 & 0.8 & 0.82 & 0.82 & 1.01 & 0.92 \\
\hline $\mathrm{La}$ & 56.88 & 55.49 & 48.64 & 47.74 & 49.16 & 48.11 & 46.77 & 50.17 & 49.25 & 48.1 & 49.81 \\
\hline $\mathrm{Ce}$ & 99.91 & 97.24 & 83.52 & 82.59 & 84.43 & 81.44 & 77.65 & 85.91 & 83.28 & 84.35 & 86.41 \\
\hline $\operatorname{Pr}$ & 10.75 & 10.41 & 8.87 & 8.79 & 9 & 8.5 & 7.99 & 8.6 & 8.23 & 8.91 & 9.31 \\
\hline $\mathrm{Sr}$ & 538 & 535 & 568 & 561 & 575 & 542 & 502 & 532 & 544 & 464 & 458 \\
\hline $\mathrm{Nd}$ & 36.42 & 35.25 & 29.57 & 29.29 & 30.04 & 28.48 & 25.72 & 28.41 & 27.13 & 30.23 & 31.45 \\
\hline $\mathrm{Zr}$ & 175 & 177 & 177 & 166 & 167 & 149 & 151 & 159 & 159 & 146 & 179 \\
\hline $\mathrm{Hf}$ & 4.74 & 4.82 & 4.91 & 4.69 & 4.6 & 4.34 & 4.64 & 4.61 & 4.58 & 4.29 & 4.86 \\
\hline Sm & 5.29 & 5.14 & 4.28 & 4.26 & 4.29 & 4.12 & 3.65 & 4.12 & 3.88 & 4.39 & 4.58 \\
\hline $\mathrm{Eu}$ & 1.25 & 1.21 & 1 & 1 & 0.99 & 1.07 & 1.01 & 1.09 & 1.07 & 1.06 & 1.05 \\
\hline Gd & 3.97 & 3.76 & 3.21 & 3.15 & 3.22 & 3.14 & 2.71 & 3.14 & 2.95 & 3.3 & 3.42 \\
\hline $\mathrm{Tb}$ & 0.43 & 0.41 & 0.36 & 0.36 & 0.37 & 0.36 & 0.3 & 0.36 & 0.33 & 0.38 & 0.4 \\
\hline Dy & 2.1 & 2 & 1.88 & 1.86 & 1.92 & 1.83 & 1.55 & 1.8 & 1.69 & 1.92 & 1.99 \\
\hline $\mathrm{Y}$ & 9.9 & 9.34 & 9.64 & 9.35 & 9.81 & 8.89 & 7.48 & 8.6 & 8.23 & 9.24 & 10.07 \\
\hline Ho & 0.38 & 0.36 & 0.36 & 0.35 & 0.36 & 0.34 & 0.29 & 0.34 & 0.31 & 0.36 & 0.37 \\
\hline $\mathrm{Er}$ & 0.98 & 0.92 & 0.97 & 0.95 & 0.99 & 0.91 & 0.77 & 0.87 & 0.83 & 0.93 & 0.98 \\
\hline $\mathrm{Tm}$ & 0.14 & 0.13 & 0.15 & 0.14 & 0.15 & 0.13 & 0.11 & 0.13 & 0.12 & 0.13 & 0.14 \\
\hline $\mathrm{Yb}$ & 0.91 & 0.83 & 0.98 & 0.93 & 0.98 & 0.86 & 0.74 & 0.81 & 0.75 & 0.89 & 0.9 \\
\hline $\mathrm{Lu}$ & 0.14 & 0.13 & 0.16 & 0.15 & 0.16 & 0.13 & 0.11 & 0.12 & 0.11 & 0.14 & 0.14 \\
\hline$\left({ }^{87} \mathrm{Sr} /{ }^{86} \mathrm{Sr}\right)_{\mathrm{i}}$ & 0.7077 & & & 0.7077 & & 0.7078 & & 0.7078 & & 0.7077 & \\
\hline$\left({ }^{143} \mathrm{Nd} /{ }^{144} \mathrm{Nd}\right)_{i}$ & 0.512322 & & & 0.512313 & & 0.512323 & & 0.512317 & & 0.512331 & \\
\hline$\varepsilon N d(t)$ & -5.1 & & & -5.3 & & -5.1 & & -5.2 & & $\begin{array}{l}-4.9 \\
\end{array}$ & \\
\hline
\end{tabular}


Table S-7 continued

\begin{tabular}{|c|c|c|c|c|c|c|c|c|c|c|c|}
\hline Samples & $\begin{array}{c}13 \mathrm{SW} \\
34-1\end{array}$ & $13 \mathrm{SW}$ & $\begin{array}{l}14 \mathrm{QW} \\
334-3\end{array}$ & $\begin{array}{l}14 \mathrm{QW} \\
33-1\end{array}$ & $14 \mathrm{QW}$ & $14 \mathrm{QW}$ & $13 \mathrm{SW}$ & $\begin{array}{c}13 \mathrm{SW} \\
44-2\end{array}$ & $13 \mathrm{SW}$ & $13 \mathrm{SW}$ & $13 \mathrm{SW}$ \\
\hline Ref. & 1 & 1 & 1 & 1 & 1 & 1 & 1 & 1 & 1 & 1 & 1 \\
\hline $\mathrm{SiO}_{2}$ & 69.52 & 69.6 & 68.98 & 69.04 & 68.99 & 69.31 & 64.98 & 64.44 & 66.05 & 65.61 & 65.13 \\
\hline $\mathrm{TiO}_{2}$ & 0.38 & 0.39 & 0.4 & 0.31 & 0.3 & 0.3 & 0.6 & 0.6 & 0.66 & 0.58 & 0.57 \\
\hline $\mathrm{Al}_{2} \mathrm{O}_{3}$ & 15.35 & 15.36 & 15.44 & 14.7 & 14.6 & 14.73 & 15.31 & 15.37 & 15.71 & 15.57 & 15.66 \\
\hline $\mathrm{Fe}_{2} \mathrm{O}_{3}$ & 3.15 & 3.06 & 3.29 & 2.3 & 2.12 & 2.19 & 3.94 & 4.12 & 4.44 & 3.74 & 3.73 \\
\hline $\mathrm{MnO}$ & 0.02 & 0.01 & 0.02 & 0.06 & 0.05 & 0.05 & 0.06 & 0.08 & 0.04 & 0.05 & 0.06 \\
\hline $\mathrm{MgO}$ & 0.32 & 0.29 & 0.38 & 0.88 & 0.89 & 0.9 & 1.22 & 1.4 & 0.65 & 1.11 & 1.09 \\
\hline $\mathrm{CaO}$ & 2.02 & 1.9 & 2.29 & 2.34 & 2.24 & 2.25 & 2.96 & 3.02 & 2.78 & 2.64 & 2.73 \\
\hline $\mathrm{Na}_{2} \mathrm{O}$ & 3.31 & 3.24 & 3.3 & 3.47 & 3.47 & 3.58 & 3.41 & 3.54 & 3.47 & 3.5 & 3.46 \\
\hline $\mathrm{K}_{2} \mathrm{O}$ & 4 & 3.97 & 3.96 & 4.52 & 4.47 & 4.38 & 4.11 & 4.03 & 4.49 & 4.13 & 4.13 \\
\hline $\mathrm{P}_{2} \mathrm{O}_{5}$ & 0.06 & 0.06 & 0.05 & 0.08 & 0.07 & 0.07 & 0.21 & 0.23 & 0.24 & 0.2 & 0.19 \\
\hline Mg\# & 17 & 16 & 19 & 43 & 45 & 45 & 38 & 40 & 23 & 37 & 37 \\
\hline $\mathrm{Cr}$ & 82 & 68 & 76 & 294 & 155 & 192 & 143 & 267 & 214 & 147 & 141 \\
\hline $\mathrm{Ni}$ & 28.2 & 25.5 & 29.9 & 27.8 & 20.9 & 19.9 & 16.9 & 27.1 & 29.1 & 17.6 & 24.7 \\
\hline $\mathrm{Rb}$ & 201 & 199 & 193 & 232 & 223 & 225 & 142 & 158 & 142 & 158 & 155 \\
\hline $\mathrm{Ba}$ & 1247 & 1173 & 1201 & 1306 & 1265 & 1285 & 1802 & 2239 & 1743 & 1953 & 1957 \\
\hline Th & 27.3 & 27 & 26.4 & 29.8 & 27.1 & $\begin{array}{l}70.1 \\
\end{array}$ & 23.8 & 25.7 & 22.7 & 25.3 & 26.2 \\
\hline $\mathrm{Nb}$ & 6 & 5.8 & 5.6 & 7.7 & 7.3 & 6.9 & 11.3 & 12.6 & 9.9 & 12.1 & 12 \\
\hline $\mathrm{Ta}$ & 0.5 & 0.49 & 0.46 & 0.72 & 0.68 & 0.66 & 0.91 & 0.96 & 0.9 & 0.96 & 0.95 \\
\hline $\mathrm{La}$ & 43.69 & 45.95 & 40.72 & 42.38 & 35.07 & 39.65 & 80.29 & 89.01 & 67.53 & 80.57 & 90.84 \\
\hline $\mathrm{Ce}$ & 79.16 & 80.41 & 72.21 & 74.55 & 60.52 & 69 & 149 & 159.3 & 125.4 & 143.3 & 160.9 \\
\hline $\operatorname{Pr}$ & 8.28 & 8.52 & 8.01 & 7.67 & 6.56 & 7.09 & 15.93 & 17.14 & 12.91 & 15.2 & 16.92 \\
\hline Sr & 376 & 352 & 420 & 428 & 409 & 410 & 1144 & 1293 & 1058 & 1314 & 1327 \\
\hline $\mathrm{Nd}$ & 29.42 & 29.6 & 28.74 & 25.91 & 22.31 & 23.88 & 52.06 & 57.33 & 42.6 & 49.7 & 54.93 \\
\hline $\mathrm{Zr}$ & 159 & 160 & 160 & 148 & 145 & 139 & 185 & 161 & 182 & 180 & 139 \\
\hline $\mathrm{Hf}$ & 4.63 & 4.52 & 4.54 & 4.45 & 4.41 & 4.16 & 5.24 & 4.37 & 5.08 & 4.76 & 3.87 \\
\hline Sm & 4.55 & 4.47 & 4.62 & 3.75 & 3.35 & 3.42 & 6.96 & 7.49 & 5.72 & 6.33 & 6.79 \\
\hline $\mathrm{Eu}$ & 1.02 & 1.01 & 1.05 & 0.81 & 0.75 & 0.75 & 1.53 & 1.67 & 1.49 & 1.49 & 1.54 \\
\hline $\mathrm{Gd}$ & 3.57 & 3.42 & 3.77 & 2.86 & 2.61 & 2.59 & 4.8 & 5.25 & 4.06 & 4.56 & 4.8 \\
\hline $\mathrm{Tb}$ & 0.41 & 0.39 & 0.45 & 0.32 & 0.29 & 0.29 & 0.52 & 0.57 & 0.44 & 0.47 & 0.49 \\
\hline Dy & 2.11 & 2 & 2.51 & 1.61 & 1.5 & 1.44 & 2.7 & 2.89 & 2.24 & 2.36 & 2.47 \\
\hline $\mathrm{Y}$ & 9.94 & 9.61 & 15.28 & 7.97 & 7.41 & 7.09 & 12.67 & 14.17 & 10.71 & 11.83 & 12.28 \\
\hline Hо & 0.4 & 0.37 & 0.52 & 0.3 & 0.28 & 0.27 & 0.5 & 0.55 & 0.42 & 0.44 & 0.47 \\
\hline Er & 1.02 & 0.95 & 1.51 & 0.78 & 0.74 & 0.71 & 1.35 & 1.46 & 1.12 & 1.2 & 1.25 \\
\hline $\mathrm{Tm}$ & 0.15 & 0.14 & 0.23 & 0.11 & 0.11 & 0.1 & 0.2 & 0.21 & 0.17 & 0.18 & 0.19 \\
\hline $\mathrm{Yb}$ & 0.94 & 0.9 & 1.58 & 0.74 & 0.7 & 0.67 & 1.29 & 1.35 & 1.07 & 1.18 & 1.2 \\
\hline $\mathrm{Lu}$ & 0.14 & 0.13 & 0.26 & 0.12 & 0.11 & 0.1 & 0.2 & 0.21 & 0.16 & 0.19 & 0.18 \\
\hline$\left({ }^{87} \mathrm{Sr} /{ }^{88} \mathrm{Sr}\right)_{\mathrm{i}}$ & & & & & & & & 0.7076 & 0.7078 & & \\
\hline$\left({ }^{143} \mathrm{Nd} /{ }^{144} \mathrm{Nd}\right)_{\mathrm{i}}$ & & & & & & & & 0.512291 & 0.51228 & & \\
\hline$\varepsilon N d(t)$ & & & & & & & & -5.7 & -5.9 & & \\
\hline
\end{tabular}


Table S. 7-continued

\begin{tabular}{|c|c|c|c|c|c|c|c|c|c|c|}
\hline Samples & $\begin{array}{c}13 \mathrm{SW} \\
45-3\end{array}$ & $\begin{array}{c}13 \mathrm{SW} \\
45-4\end{array}$ & $\begin{array}{c}13 \mathrm{SW} \\
45-5\end{array}$ & $\begin{array}{c}\text { LQS } \\
-2\end{array}$ & $\begin{array}{c}\text { LQS } \\
-3\end{array}$ & $\begin{array}{c}\text { LQS } \\
-6\end{array}$ & $\begin{array}{c}\text { LQS } \\
-7\end{array}$ & $\begin{array}{c}\text { LQS } \\
-14\end{array}$ & $\begin{array}{c}\text { D50 } \\
85\end{array}$ & $\begin{array}{c}\text { D50 } \\
87\end{array}$ \\
\hline Ref. & 1 & 1 & 1 & 2 & 2 & 2 & 2 & 2 & 2 & 2 \\
\hline $\mathrm{SiO}_{2}$ & 64.96 & 65.33 & 64.96 & 61.76 & 63.29 & 58.05 & 57.53 & 67.7 & 58.59 & 62.01 \\
\hline $\mathrm{TiO}_{2}$ & 0.59 & 0.54 & 0.56 & 0.51 & 0.55 & 0.77 & 0.78 & 0.45 & 0.79 & 0.84 \\
\hline $\mathrm{Al}_{2} \mathrm{O}_{3}$ & 15.42 & 15.49 & 15.35 & 14.87 & 15.03 & 15.49 & 15.56 & 15.96 & 15.22 & 15.4 \\
\hline $\mathrm{Fe}_{2} \mathrm{O}_{3}$ & 3.83 & 3.81 & 3.86 & 4.81 & 4.84 & 6.19 & 5.97 & 3.34 & 7.2 & 5.33 \\
\hline $\mathrm{MnO}$ & 0.07 & 0.07 & 0.07 & 0.07 & 0.04 & 0.09 & 0.1 & 0.05 & 0.09 & 0.07 \\
\hline $\mathrm{MgO}$ & 1.43 & 1.16 & 1.3 & 5.44 & 4.02 & 4.67 & 6.09 & 1.67 & 5.74 & 4.43 \\
\hline $\mathrm{CaO}$ & 2.85 & 2.86 & 3.07 & 5.21 & 4.69 & 7.75 & 7.11 & 3.19 & 6.35 & 4.53 \\
\hline $\mathrm{Na}_{2} \mathrm{O}$ & 3.45 & 3.65 & 3.56 & 3.95 & 3.96 & 4.03 & 3.9 & 4.26 & 3.04 & 2.98 \\
\hline $\mathrm{K}_{2} \mathrm{O}$ & 4 & 4.02 & 4 & 3.32 & 3.53 & 2.5 & 2.47 & 3.25 & 2.93 & 4.33 \\
\hline $\mathrm{P}_{2} \mathrm{O}_{5}$ & 0.21 & 0.22 & 0.21 & 0.06 & 0.06 & 0.47 & 0.49 & 0.15 & 0.04 & 0.08 \\
\hline $\mathrm{Mg} \#$ & 43 & 38 & 40 & 69 & 62 & 60 & 67 & 50 & 61 & 62 \\
\hline $\mathrm{Cr}$ & 138 & 211 & 198 & 212 & 253 & 209 & 211 & 33.7 & 368 & 176 \\
\hline $\mathrm{Ni}$ & 17.1 & 29 & 23.2 & 146 & 125 & 150 & 152 & 23.9 & 173 & 92.2 \\
\hline $\mathrm{Rb}$ & 156 & 161 & 95 & 84.2 & 95.1 & 56 & 56.6 & 98.8 & 84.6 & 157 \\
\hline $\mathrm{Ba}$ & 1876 & 1961 & 1840 & 1741 & 1880 & 1520 & 1604 & 1296 & 1957 & 1923 \\
\hline Th & 25.4 & 24.9 & 15 & 17 & 15.8 & 14.9 & 16 & 19.7 & 13.4 & 29.1 \\
\hline $\mathrm{Nb}$ & 11.9 & 12 & 12 & 7.56 & 8.31 & 11.9 & 12.4 & 4.9 & 9.61 & 14.5 \\
\hline $\mathrm{Ta}$ & 0.91 & 0.95 & 0.92 & 0.47 & 0.5 & 0.69 & 0.73 & 0.33 & 0.66 & 1.14 \\
\hline $\mathrm{La}$ & 90.03 & 80.52 & 53.46 & 56.1 & 47.7 & 73.1 & 76.9 & 52.2 & 45 & 69.8 \\
\hline $\mathrm{Ce}$ & 159.5 & 145.2 & 100.2 & 92.2 & 70 & 128 & 134 & 90 & 92.9 & 118 \\
\hline Pr & 17.07 & 15.51 & 10.34 & 10.3 & 7.59 & 14.5 & 15.5 & 10.1 & 7.98 & 12.9 \\
\hline $\mathrm{Sr}$ & 1234 & 1280 & 965 & 2276 & 2113 & 2118 & 2256 & 816 & 1876 & 1552 \\
\hline $\mathrm{Nd}$ & 56.57 & 50.98 & 36.45 & 36.6 & 26.4 & 51.2 & 54.9 & 34.7 & 28.7 & 43.6 \\
\hline $\mathrm{Zr}$ & 151 & 164 & 171 & 183 & 199 & 217 & 223 & 239 & 211 & 299 \\
\hline $\mathrm{Hf}$ & 4.05 & 4.44 & 4.57 & 4.45 & 4.79 & 4.68 & 5 & 5.8 & 5.11 & 6.71 \\
\hline $\mathrm{Sm}$ & 7.21 & 6.62 & 5.34 & 5.39 & 3.93 & 7.34 & 7.97 & 5.07 & 4.66 & 6.13 \\
\hline $\mathrm{Eu}$ & 1.54 & 1.54 & 1.23 & 1.45 & 1.7 & 1.79 & 1.84 & 1.08 & 1.21 & 1.6 \\
\hline $\mathrm{Gd}$ & 5.17 & 4.76 & 4.18 & 2.77 & 2.13 & 4.34 & 4.44 & 2.86 & 3.1 & 3.44 \\
\hline $\mathrm{Tb}$ & 0.54 & 0.5 & 0.47 & 0.41 & 0.34 & 0.66 & 0.67 & 0.41 & 0.45 & 0.49 \\
\hline Dy & 2.79 & 2.55 & 2.48 & 1.82 & 1.65 & 3.27 & 3.24 & 1.8 & 2.45 & 2.42 \\
\hline $\mathrm{Y}$ & 13.87 & 12.81 & 13.02 & 8.99 & 7.85 & 16.6 & 16.9 & 8.49 & 12 & 11.7 \\
\hline Ho & 0.53 & 0.49 & 0.48 & 0.32 & 0.29 & 0.59 & 0.61 & 0.33 & 0.49 & 0.43 \\
\hline $\mathrm{Er}$ & 1.4 & 1.32 & 1.32 & 0.84 & 0.75 & 1.45 & 1.52 & 0.81 & 1.24 & 1.14 \\
\hline $\mathrm{Tm}$ & 0.2 & 0.19 & 0.19 & 0.13 & 0.12 & 0.21 & 0.22 & 0.12 & 0.19 & 0.15 \\
\hline $\mathrm{Yb}$ & 1.31 & 1.26 & 1.26 & 0.89 & 0.83 & 1.45 & 1.45 & 0.79 & 1.21 & 0.96 \\
\hline $\mathrm{Lu}$ & 0.2 & 0.19 & 0.19 & 0.14 & 0.14 & 0.24 & 0.24 & 0.13 & 0.19 & 0.15 \\
\hline$\left({ }^{87} \mathrm{Sr} /{ }^{86} \mathrm{Sr}\right)_{\mathrm{i}}$ & 0.7076 & 0.7076 & & 0.7065 & 0.7067 & 0.7068 & 0.7067 & 0.7072 & 0.7063 & 0.7070 \\
\hline$\left({ }^{143} \mathrm{Nd} /{ }^{144} \mathrm{Nd}\right)_{\mathrm{i}}$ & 0.512295 & 0.51228 & & 0.512461 & 0.512454 & 0.51244 & 0.512463 & 0.512445 & 0.51237 & 0.512382 \\
\hline$\varepsilon \mathrm{Nd}(\mathrm{t})$ & -5.6 & -5.9 & & -3.45 & -3.59 & -3.87 & -3.41 & -3.77 & -5.23 & -5 \\
\hline
\end{tabular}


Table S. 7-continued

\begin{tabular}{|c|c|c|c|c|c|c|c|c|c|c|}
\hline Samples & $\begin{array}{c}\text { D50 } \\
96\end{array}$ & $\begin{array}{c}\text { D5079 } \\
-1\end{array}$ & $\begin{array}{c}\text { D513 } \\
6-3\end{array}$ & $\begin{array}{l}\text { D51 } \\
37-2\end{array}$ & $\begin{array}{c}9013 \\
-1\end{array}$ & $\begin{array}{c}5079- \\
5-1\end{array}$ & $7509-1$ & Z19H5 & Z19H6 & $\mathrm{Z} 08 \mathrm{H} 1$ \\
\hline Ref. & 2 & 2 & 2 & 2 & 4 & 4 & 4 & 3 & 3 & 3 \\
\hline $\mathrm{SiO}_{2}$ & 63.03 & 65.32 & 61.31 & 58.6 & 62 & 62.35 & 59.78 & 65.27 & 64.99 & 62.54 \\
\hline $\mathrm{TiO}_{2}$ & 0.83 & 0.71 & 0.92 & 0.91 & 0.79 & 0.74 & 1.13 & 0.4 & 0.39 & 0.69 \\
\hline $\mathrm{Al}_{2} \mathrm{O}_{3}$ & 15.9 & 14.76 & 16 & 16.85 & 15.3 & 15.14 & 15.15 & 15.13 & 14.95 & 15.3 \\
\hline $\mathrm{Fe}_{2} \mathrm{O}_{3}$ & 4.72 & 4.47 & 5.73 & 5.79 & 5.04 & 4.96 & 5.57 & 3.07 & 3.07 & 4.18 \\
\hline $\mathrm{MnO}$ & 0.06 & 0.06 & 0.09 & 0.12 & 0.11 & 0.07 & 0.07 & 0.05 & 0.05 & 0.06 \\
\hline $\mathrm{MgO}$ & 3.57 & 3.72 & 4.12 & 4.29 & 4.18 & 4.31 & 4.66 & 2.5 & 2.51 & 3.79 \\
\hline $\mathrm{CaO}$ & 4.31 & 3.69 & 5.03 & 6.47 & 4.46 & 4.49 & 4.38 & 3.51 & 3.51 & 4.52 \\
\hline $\mathrm{Na}_{2} \mathrm{O}$ & 3.34 & 2.66 & 3.19 & 3.47 & 2.86 & 2.83 & 3.06 & 3.68 & 3.59 & 3.61 \\
\hline $\mathrm{K}_{2} \mathrm{O}$ & 4.17 & 4.52 & 3.4 & 3.43 & 4.41 & 3.92 & 4.96 & 3.75 & 3.76 & 3.3 \\
\hline $\mathrm{P}_{2} \mathrm{O}_{5}$ & 0.09 & 0.09 & 0.21 & 0.06 & 0.07 & 0.1 & 0.04 & 0.17 & 0.18 & 0.31 \\
\hline $\mathrm{Mg} \#$ & 60 & 62 & 59 & 60 & 62 & 63 & 63 & 62 & 62 & 64 \\
\hline $\mathrm{Cr}$ & 159 & 151 & 162 & 307 & 162 & 178 & 196 & 69.2 & 71.4 & 243 \\
\hline $\mathrm{Ni}$ & 82 & 73.6 & 85.8 & 97.5 & 94.5 & 94.9 & 118 & 43.1 & 42.9 & 148 \\
\hline $\mathrm{Rb}$ & 158 & 181 & 113 & 86.3 & 159 & 151 & 194 & 153 & 153 & 115 \\
\hline $\mathrm{Ba}$ & 2255 & 1844 & 1290 & 1447 & 2153 & 2128 & 1699 & 1161 & 1172 & 1732 \\
\hline Th & 23.6 & 22.7 & 15.8 & 11.2 & 25.4 & 23.6 & 14 & 14.9 & 14.9 & 24 \\
\hline $\mathrm{Nb}$ & 13.6 & 12.7 & 9.17 & 9.11 & 12.7 & 12.9 & 13.3 & 6.12 & 6.19 & 8.79 \\
\hline $\mathrm{Ta}$ & 0.96 & 0.98 & 0.65 & 0.65 & 0.92 & 0.94 & 0.84 & 0.54 & 0.56 & 0.59 \\
\hline $\mathrm{La}$ & 60.2 & 61.9 & 43.2 & 18.1 & 68.9 & 73.9 & 24.7 & 30.7 & 30.6 & 68.2 \\
\hline $\mathrm{Ce}$ & 103 & 110 & 73.9 & 32.9 & 110 & 129 & 38.8 & 54.2 & 54.3 & 123 \\
\hline $\operatorname{Pr}$ & 11.2 & 12.3 & 8.38 & 3.83 & 12 & 13.6 & 4.15 & 5.9 & 5.9 & 13 \\
\hline $\mathrm{Sr}$ & 1747 & 1095 & 917 & 1281 & 1601 & 1607 & 1496 & 728 & 716 & 1151 \\
\hline $\mathrm{Nd}$ & 38.4 & 41.4 & 30.2 & 15.1 & 41.5 & 46.6 & 15.2 & 21.4 & 21.7 & 48.9 \\
\hline $\mathrm{Zr}$ & 306 & 282 & 228 & 231 & 271 & 279 & 431 & 138 & 144 & 204 \\
\hline $\mathrm{Hf}$ & 7.09 & 6.54 & 5.41 & 5.51 & 6.33 & 6.6 & 10.6 & 3.66 & 3.79 & 5.08 \\
\hline $\mathrm{Sm}$ & 5.22 & 5.93 & 4.82 & 2.8 & 5.86 & 6.45 & 2.92 & 3.57 & 3.68 & 7.36 \\
\hline $\mathrm{Eu}$ & 1.66 & 1.43 & 1.41 & 1.39 & 1.52 & 1.56 & 1.6 & 0.94 & 0.96 & 1.82 \\
\hline Gd & 2.84 & 3.38 & 3.16 & 2.17 & 3.09 & 3.78 & 2.12 & 2.84 & 2.94 & 5.79 \\
\hline $\mathrm{Tb}$ & 0.43 & 0.54 & 0.47 & 0.31 & 0.45 & 0.51 & 0.33 & 0.38 & 0.38 & 0.68 \\
\hline Dy & 2 & 2.68 & 2.44 & 1.74 & 2.23 & 2.56 & 1.79 & 1.84 & 1.92 & 3.37 \\
\hline $\mathrm{Y}$ & 9.58 & 13.2 & 11.8 & 7.98 & 10.6 & 12.8 & 8.58 & 11 & 11.1 & 17.7 \\
\hline Ho & 0.35 & 0.5 & 0.44 & 0.32 & 0.4 & 0.46 & 0.34 & 0.37 & 0.37 & 0.6 \\
\hline $\mathrm{Er}$ & 0.93 & 1.32 & 1.13 & 0.84 & 1.04 & 1.24 & 0.84 & 0.94 & 0.95 & 1.62 \\
\hline $\mathrm{Tm}$ & 0.12 & 0.19 & 0.16 & 0.12 & 0.14 & 0.17 & 0.14 & 0.15 & 0.15 & 0.24 \\
\hline $\mathrm{Yb}$ & 0.79 & 1.17 & 1.01 & 0.76 & 0.86 & 1.05 & 1.01 & 0.93 & 0.94 & 1.43 \\
\hline $\mathrm{Lu}$ & 0.13 & 0.19 & 0.16 & 0.13 & 0.13 & 0.17 & 0.16 & 0.14 & 0.14 & 0.21 \\
\hline$\left({ }^{87} \mathrm{Sr} /{ }^{86} \mathrm{Sr}\right)_{\mathrm{i}}$ & 0.7071 & 0.7077 & 0.7077 & & 0.7070 & 0.7072 & 0.7068 & & & 0.7073 \\
\hline$\left({ }^{143} \mathrm{Nd} /{ }^{144} \mathrm{Nd}\right)_{i}$ & 0.51236 & 0.512287 & 0.512369 & & 0.512408 & 0.512353 & 0.512484 & & & 0.512369 \\
\hline$\varepsilon \mathrm{Nd}(\mathrm{t})$ & -5.42 & -6.84 & -5.24 & & -4.49 & -5.56 & -3 & & & -5.25 \\
\hline
\end{tabular}


Table S7-continued

\begin{tabular}{|c|c|c|c|c|c|c|c|c|c|c|c|c|}
\hline Samples & D5139 & $\begin{array}{c}\text { D51 } \\
40\end{array}$ & D5141 & $\begin{array}{c}\text { D51 } \\
3 \\
0-2\end{array}$ & $\begin{array}{c}\text { D513 } \\
0-3\end{array}$ & $\begin{array}{c}\text { D513 } \\
1-1\end{array}$ & $\begin{array}{c}\text { D513 } \\
2-1\end{array}$ & $\begin{array}{c}\text { D513 } \\
2-2\end{array}$ & $\begin{array}{l}\text { D51 } \\
35-1\end{array}$ & $\begin{array}{l}\text { D51 } \\
35-2\end{array}$ & $\begin{array}{l}\text { D51 } \\
35-3\end{array}$ & $\begin{array}{c}\text { D513 } \\
6-1\end{array}$ \\
\hline Reference & 2 & 2 & 2 & 2 & 2 & 2 & 2 & 2 & 2 & 2 & 2 & 2 \\
\hline $\mathrm{SiO}_{2}$ & 63.17 & 66.16 & $\begin{array}{l}62.19 \\
\end{array}$ & 56.1 & 61.73 & 62.8 & 64.4 & 64.28 & 61.29 & 63.82 & 65.17 & 60.86 \\
\hline $\mathrm{TiO}_{2}$ & 0.85 & 0.56 & 0.68 & 1.03 & 0.75 & 0.84 & 0.69 & 0.69 & 0.88 & 0.8 & 0.7 & 0.87 \\
\hline $\mathrm{Al}_{2} \mathrm{O}_{3}$ & 15.96 & 15.41 & 14.69 & 15.4 & 15.69 & 16.23 & 16.09 & 16.25 & 16.13 & 16.21 & 16.02 & 16.1 \\
\hline $\mathrm{Fe}_{2} \mathrm{O}_{3}$ & 5.7 & 3.89 & 5.27 & 7.16 & 5.61 & 5.25 & 4.74 & 4.97 & 5.76 & 5.32 & 4.42 & 5.81 \\
\hline $\mathrm{MnO}$ & 0.09 & 0.04 & 0.06 & 0.1 & 0.08 & 0.08 & 0.03 & 0.03 & 0.21 & 0.05 & 0.06 & 0.18 \\
\hline $\mathrm{MgO}$ & 2.8 & 2.88 & 5.87 & 6.08 & 4.54 & 3.4 & 1.99 & 1.84 & 3.43 & 2.61 & 2.91 & 3.8 \\
\hline $\mathrm{CaO}$ & 4.53 & 4.07 & 4.96 & 6.94 & 4.71 & 4.48 & 4.37 & 4.15 & 5.55 & 4.18 & 3.86 & 5.58 \\
\hline $\mathrm{Na}_{2} \mathrm{O}$ & 3.46 & 3.36 & 3.19 & 3.52 & 3.27 & 3.54 & 4.51 & 4.57 & 3.49 & 3.51 & 3.35 & 3.5 \\
\hline $\mathrm{K}_{2} \mathrm{O}$ & 3.31 & 3.58 & 3.05 & 3.4 & 3.55 & 3.33 & 3.13 & 3.18 & 3.18 & 3.35 & 3.49 & 3.15 \\
\hline $\mathrm{P}_{2} \mathrm{O}_{5}$ & $\begin{array}{l}0.14 \\
\end{array}$ & 0.05 & 0.05 & 0.25 & 0.08 & 0.05 & 0.04 & 0.04 & 0.07 & 0.14 & 0.03 & 0.16 \\
\hline Mg\# & 50 & 60 & 69 & 63 & 62 & 56 & 46 & 43 & 54 & 50 & 57 & 57 \\
\hline $\mathrm{Cr}$ & 235 & 169 & 383 & 362 & 206 & 116 & 121 & 127 & 224 & 132 & 110 & 239 \\
\hline $\mathrm{Ni}$ & 86.2 & 103 & 183 & 144 & 101 & 67.3 & 59.3 & 56.6 & 87.5 & 72.7 & 56 & 110.5 \\
\hline $\mathrm{Rb}$ & 127 & 117 & 90.4 & 86.6 & 118 & 100 & 98.5 & 103 & 94.1 & 107 & 109 & 99.4 \\
\hline $\mathrm{Ba}$ & 1300 & 1483 & 1431 & 1786 & 1838 & 1757 & 1968 & 2064 & 1618 & 1356 & 1400 & 1563 \\
\hline Th & 17.7 & 17.5 & 16.4 & 22.4 & 23 & 19.1 & 13.1 & 13.3 & 14.7 & 16.1 & 17.3 & 15.2 \\
\hline $\mathrm{Nb}$ & 7.07 & 8.48 & 7.25 & 14.8 & 9.81 & 10.6 & 7.78 & 7.47 & 9.9 & 9.33 & 8.85 & 9.81 \\
\hline $\mathrm{Ta}$ & 0.52 & 0.57 & 0.47 & 0.85 & 0.59 & 0.65 & 0.5 & 0.51 & 0.64 & 0.6 & 0.59 & 0.65 \\
\hline $\mathrm{La}$ & 33.7 & 50.1 & 39.3 & 61.8 & 52.4 & 43.9 & 25.1 & 27.3 & 31.2 & 26.7 & 33.2 & 35.9 \\
\hline $\mathrm{Ce}$ & 58 & 92.7 & 66.2 & 112 & 88.7 & 69.7 & 45.8 & 43.9 & 53.8 & 46.6 & 55 & 66.2 \\
\hline $\mathrm{Pr}$ & 6.1 & 10.4 & 6.79 & 13 & 9.37 & 7.25 & 4.75 & 5.16 & 5.61 & 5.02 & 5.56 & 7.32 \\
\hline $\mathrm{Sr}$ & 851 & 1220 & 1443 & 1669 & 1135 & 1073 & 959 & 1030 & 1188 & 892 & 878 & 1191 \\
\hline $\mathrm{Nd}$ & 20.8 & 35.1 & 22.7 & 47.3 & 31.1 & 24.3 & 17.2 & 19.2 & 20.5 & 17.8 & 17.9 & 26.6 \\
\hline $\mathrm{Zr}$ & 180 & 217 & 198 & 305 & 263 & 273 & 206 & 198 & 218 & 221 & 222 & 221 \\
\hline $\mathrm{Hf}$ & 4.46 & 5.27 & 4.66 & 6.87 & 5.93 & 5.86 & 5.09 & 4.85 & 5.31 & 5.39 & 5.56 & 5.2 \\
\hline $\mathrm{Sm}$ & 3.18 & 5.13 & 3.53 & 7.11 & 4.4 & 3.46 & 3.01 & 3.15 & 3.39 & 2.78 & 2.7 & 4.09 \\
\hline $\mathrm{Eu}$ & 1 & 1.23 & 1.33 & 2.16 & 1.38 & 1.38 & 1.02 & 1.15 & 1.39 & 1.16 & 1.15 & 1.51 \\
\hline $\mathrm{Gd}$ & 1.97 & 2.86 & 2.26 & 4.16 & 2.5 & 2.06 & 2.04 & 2.19 & 2.34 & 1.76 & 1.63 & 2.6 \\
\hline $\mathrm{Tb}$ & 0.32 & 0.41 & 0.36 & 0.62 & 0.39 & 0.32 & 0.32 & 0.32 & 0.35 & 0.27 & 0.26 & 0.41 \\
\hline Dy & 1.7 & 2.08 & 1.97 & 3.16 & 1.99 & 1.66 & 1.74 & 1.72 & 2 & 1.47 & 1.44 & 2.15 \\
\hline $\mathrm{Y}$ & 8.82 & 10.3 & 9.77 & 15.4 & 9.89 & 8.07 & 8.47 & 8.36 & 9.61 & 6.63 & 6.96 & 10.3 \\
\hline Ho & 0.33 & 0.38 & 0.38 & 0.6 & 0.37 & 0.31 & 0.34 & 0.34 & 0.39 & 0.28 & 0.28 & 0.41 \\
\hline Er & 0.93 & 1.01 & 1 & 1.51 & 0.99 & 0.8 & 0.88 & 0.87 & 0.99 & 0.7 & 0.74 & 1.07 \\
\hline $\mathrm{Tm}$ & 0.14 & 0.15 & 0.15 & 0.21 & 0.14 & 0.12 & 0.13 & 0.13 & 0.15 & 0.1 & 0.11 & 0.15 \\
\hline $\mathrm{Yb}$ & 0.87 & 0.91 & 0.94 & 1.33 & 0.91 & 0.73 & 0.81 & 0.81 & 0.94 & 0.63 & 0.72 & 0.92 \\
\hline $\mathrm{Lu}$ & 0.14 & 0.14 & 0.15 & 0.2 & 0.15 & 0.12 & 0.13 & 0.13 & 0.15 & 0.1 & 0.12 & 0.15 \\
\hline$\left({ }^{87} \mathrm{Sr}^{86}{ }^{86} \mathrm{r}_{\mathrm{i}}\right.$ & & 0.7069 & & & & 0.7073 & & 0.7070 & & & & \\
\hline$\left({ }^{1+3} \mathrm{Nd} / /^{1 / 4} \mathrm{Nd}\right)_{i}$ & & $\begin{array}{c}0.51243 \\
6 \\
\end{array}$ & & & & 0.512386 & & 0.512434 & & & & \\
\hline$\varepsilon \mathrm{Nd}(\mathrm{t})$ & & $\begin{array}{l}-3.94 \\
\end{array}$ & & & & -4.92 & & -3.98 & & & & \\
\hline
\end{tabular}

Table S7-continued

\begin{tabular}{|c|c|c|c|c|c|c|c|c|c|c|}
\hline Samples & $\begin{array}{c}\text { D5144- } \\
2\end{array}$ & $\begin{array}{c}\text { D5144- } \\
3\end{array}$ & $\begin{array}{c}\text { D5144- } \\
4\end{array}$ & $\begin{array}{c}\text { D5144- } \\
5\end{array}$ & D5144-7 & 3118 & $\begin{array}{c}\text { LQS } \\
-7\end{array}$ & $\begin{array}{c}7509 \\
-1\end{array}$ & $\begin{array}{c}9020 \\
-2\end{array}$ & $\begin{array}{c}9020 \\
-6\end{array}$ \\
\hline Reference & 2 & 2 & 2 & 2 & 2 & 4 & 4 & 4 & 4 & 4 \\
\hline $\mathrm{SiO}_{2}$ & 60.29 & 63.52 & 64.32 & 64.58 & 60.57 & 61.01 & 57.31 & 59.78 & 64.85 & 60.13 \\
\hline $\mathrm{TiO}_{2}$ & 0.89 & 0.55 & 0.61 & 0.61 & 0.83 & 0.58 & 0.78 & 1.13 & 0.6 & 0.92 \\
\hline $\mathrm{Al}_{2} \mathrm{O}_{3}$ & 16.35 & 15.54 & 15.46 & 15.45 & 15.93 & 15.28 & 15.5 & 15.15 & 14.88 & 14.67 \\
\hline $\mathrm{Fe}_{2} \mathrm{O}_{3}$ & 5.93 & 4.83 & 4.48 & 4.43 & 5.53 & 4.83 & 5.95 & 5.57 & 4.06 & 5.45 \\
\hline $\mathrm{MnO}$ & 0.07 & 0.06 & 0.17 & 0.08 & 0.05 & 0.07 & 0.1 & 0.07 & 0.06 & 0.1 \\
\hline
\end{tabular}




\begin{tabular}{|c|c|c|c|c|c|c|c|c|c|c|}
\hline $\mathrm{MgO}$ & 3.89 & 4.02 & 3.39 & 3.3 & 3.7 & 4.37 & 6.07 & 4.66 & 2.54 & 4.34 \\
\hline $\mathrm{CaO}$ & 5.89 & 4.16 & 4.24 & 4.2 & 5.79 & 6.15 & 7.09 & 4.38 & 3.48 & 5.8 \\
\hline $\mathrm{Na}_{2} \mathrm{O}$ & 3.16 & 3.73 & 3.61 & 3.64 & 3.46 & 3.76 & 3.89 & 3.06 & 2.59 & 2.89 \\
\hline $\mathrm{K}_{2} \mathrm{O}$ & 3.33 & 3.55 & 3.59 & 3.63 & 4.12 & 3.61 & 2.46 & 4.96 & 4.65 & 4.63 \\
\hline $\mathrm{P}_{2} \mathrm{O}_{5}$ & 0.2 & 0.03 & 0.13 & 0.08 & 0.02 & 0.21 & 0.48 & 0.04 & 0.23 & 0.45 \\
\hline $\mathrm{Mg} \#$ & 57 & 62 & 60 & 60 & 57 & 64 & 67 & 63 & 56 & 61 \\
\hline $\mathrm{Cr}$ & 291 & 194 & 183 & 171 & 206 & 108 & 211 & 196 & 79 & 228 \\
\hline $\mathrm{Ni}$ & 104 & 124 & 132 & 121 & 88 & 101 & 152 & 118 & 33.4 & 137 \\
\hline $\mathrm{Rb}$ & 109 & 124 & 127 & 114 & 105 & 81.5 & 56.6 & 194 & 184 & 142 \\
\hline $\mathrm{Ba}$ & 1358 & 1554 & 1952 & 1672 & 2120 & 1676 & 1604 & 1699 & 1502 & 2635 \\
\hline Th & 16.1 & 11.6 & 19.2 & 17.8 & 13.4 & 16.1 & 16 & 14 & 30.2 & 21.2 \\
\hline $\mathrm{Nb}$ & 9.53 & 6.71 & 7.57 & 7.72 & 8.9 & 6.06 & 12.4 & 13.3 & 13.2 & 12.7 \\
\hline $\mathrm{Ta}$ & 0.67 & 0.47 & 0.5 & 0.51 & 0.53 & 0.48 & 0.73 & 0.84 & 1.18 & 0.76 \\
\hline $\mathrm{La}$ & 41.6 & 31.4 & 50.6 & 42.4 & 49.2 & 56 & 76.9 & 24.7 & 77.4 & 87.1 \\
\hline $\mathrm{Ce}$ & 75.6 & 47.4 & 91.1 & 78 & 81.6 & 106 & 134 & 38.8 & 133 & 157 \\
\hline Pr & 8.94 & 4.94 & 9.7 & 8.24 & 8.3 & 13 & 15.5 & 4.15 & 14.6 & 19.1 \\
\hline $\mathrm{Sr}$ & 1152 & 1301 & 1519 & 1481 & 1907 & 2472 & 2256 & 1496 & 1085 & 2000 \\
\hline $\mathrm{Nd}$ & 31.8 & 17.9 & 33.2 & 29 & 28.3 & 48.9 & 54.9 & 15.2 & 49.2 & 69.6 \\
\hline $\mathrm{Zr}$ & 217 & 172 & 212 & 204 & 207 & 169 & 223 & 431 & 266 & 373 \\
\hline $\mathrm{Hf}$ & 5.08 & 4.4 & 5.1 & 5.03 & 4.91 & 4.7 & 5 & 10.6 & 5.94 & 8.43 \\
\hline $\mathrm{Sm}$ & 4.84 & 2.83 & 4.77 & 4.4 & 4.18 & 7.57 & 7.97 & 2.92 & 6.93 & 10 \\
\hline $\mathrm{Eu}$ & 1.44 & 1.16 & 1.27 & 1.29 & 1.44 & 1.76 & 1.84 & 1.6 & 1.44 & 2.3 \\
\hline $\mathrm{Gd}$ & 3 & 1.77 & 2.66 & 2.52 & 2.46 & 4.12 & 4.44 & 2.12 & 3.79 & 5.3 \\
\hline $\mathrm{Tb}$ & 0.47 & 0.27 & 0.39 & 0.38 & 0.35 & 0.59 & 0.67 & 0.33 & 0.59 & 0.75 \\
\hline Dy & 2.42 & 1.46 & 1.99 & 1.88 & 1.73 & 2.8 & 3.24 & 1.79 & 2.88 & 3.5 \\
\hline $\mathrm{Y}$ & 12 & 7.86 & 10.14 & 9.15 & 8.33 & 13.9 & 16.9 & 8.58 & 14.8 & 16.6 \\
\hline Ho & 0.45 & 0.29 & 0.37 & 0.34 & 0.33 & 0.54 & 0.61 & 0.34 & 0.51 & 0.61 \\
\hline $\mathrm{Er}$ & 1.22 & 0.78 & 0.97 & 0.95 & 0.86 & 1.42 & 1.52 & 0.84 & 1.31 & 1.53 \\
\hline $\mathrm{Tm}$ & 0.17 & 0.12 & 0.14 & 0.13 & 0.12 & 0.22 & 0.22 & 0.14 & 0.18 & 0.21 \\
\hline $\mathrm{Yb}$ & 1.05 & 0.85 & 0.9 & 0.85 & 0.77 & 1.48 & 1.45 & 1.01 & 1.24 & 1.37 \\
\hline $\mathrm{Lu}$ & 0.17 & 0.14 & 0.14 & 0.13 & 0.13 & 0.25 & 0.24 & 0.16 & 0.19 & 0.21 \\
\hline$\left({ }^{87} \mathrm{Sr} /{ }^{86} \mathrm{Sr}\right)_{\mathrm{i}}$ & & & & & 0.7065 & & 0.7067 & 0.7068 & 0.7078 & 0.7066 \\
\hline$\left({ }^{143} \mathrm{Nd} /{ }^{144} \mathrm{Nd}\right)_{\mathrm{i}}$ & & & & & 0.512417 & & 0.512463 & 0.512484 & 0.512327 & 0.512423 \\
\hline$\varepsilon N d(t)$ & & & & & -4.31 & & -3.41 & -3 & -6.07 & -4.19 \\
\hline
\end{tabular}

Table 7-continued

\begin{tabular}{|c|c|c|c|c|c|c|c|c|c|c|}
\hline Samples & $\begin{array}{c}5079 \\
-6-1 \\
\end{array}$ & 8540 & $\begin{array}{c}5132 \\
-4 \\
\end{array}$ & $\begin{array}{c}5130 \\
-1 \\
\end{array}$ & $\begin{array}{c}5130 \\
-4 \\
\end{array}$ & $\begin{array}{c}5130 \\
-5 \\
\end{array}$ & $\begin{array}{c}5131 \\
-2-1 \\
\end{array}$ & $\begin{array}{c}5137 \\
-1\end{array}$ & 5141 & $\begin{array}{c}5144 \\
-1 \\
\end{array}$ \\
\hline Ref. & 4 & 4 & 4 & 4 & 4 & 4 & 4 & 4 & 4 & 4 \\
\hline $\mathrm{SiO}_{2}$ & 64.48 & 59.62 & 63.13 & 55.33 & 61.96 & 61.95 & 62.9 & 57.25 & 61.58 & 63.89 \\
\hline $\mathrm{TiO}_{2}$ & 0.66 & 0.88 & 0.67 & 1.01 & 0.84 & 0.82 & 0.8 & 0.89 & 0.67 & 0.6 \\
\hline $\mathrm{Al}_{2} \mathrm{O}_{3}$ & 15.32 & 15.1 & 15.22 & 15.11 & 15.48 & 15.34 & 15.48 & 16.47 & 14.55 & 15.31 \\
\hline $\mathrm{Fe}_{2} \mathrm{O}_{3}$ & 4.24 & 5.47 & 4.89 & 7.2 & 5.41 & 5.05 & 5.07 & 6.26 & 5.22 & 4.29 \\
\hline $\mathrm{MnO}$ & 0.06 & 0.08 & 0.08 & 0.17 & 0.07 & 0.07 & 0.07 & 0.11 & 0.06 & 0.07 \\
\hline $\mathrm{MgO}$ & 3.01 & 4.33 & 2.79 & 6.34 & 3.38 & 3.29 & 3.31 & 4.99 & 5.82 & 3.51 \\
\hline $\mathrm{CaO}$ & 3.92 & 6.24 & 4.72 & 6.64 & 4.88 & 4.7 & 4.54 & 6.39 & 4.91 & 4.21 \\
\hline $\mathrm{Na}_{2} \mathrm{O}$ & 3.08 & 2.83 & 3.74 & 3.12 & 3.65 & 3.54 & 3.82 & 3.35 & 3.15 & 3.58 \\
\hline $\mathrm{K}_{2} \mathrm{O}$ & 4.14 & 4.07 & 3.15 & 3.34 & 3.12 & 3.5 & 3.46 & 3.35 & 3.02 & 3.51 \\
\hline $\mathrm{P}_{2} \mathrm{O}_{5}$ & 0.16 & 0.27 & 0.2 & 0.28 & 0.24 & 0.27 & 0.3 & 0.09 & 0.05 & 0.16 \\
\hline $\mathrm{Mg} \#$ & 59 & 61 & 53 & 64 & 56 & 57 & 57 & 61 & 69 & 62 \\
\hline $\mathrm{Cr}$ & 116 & 215 & 135 & 354 & 109 & 114 & 103 & 314 & 383 & 168 \\
\hline $\mathrm{Ni}$ & 54 & 105 & 82.2 & 154 & 67.1 & 65.1 & 62.8 & 119 & 183 & 114 \\
\hline $\mathrm{Rb}$ & 142 & 150 & 112 & 88.3 & 100 & 109 & 113 & 90.3 & 90.4 & 122 \\
\hline $\mathrm{Ba}$ & 1803 & 2329 & 1686 & 1845 & 1805 & 1715 & 1541 & 1403 & 1431 & 1705 \\
\hline Th & 21.6 & 21.1 & 18 & 21.2 & 23.8 & 24.7 & 26 & 12.3 & 16.4 & 18.6 \\
\hline $\mathrm{Nb}$ & 10.6 & 13.6 & 8.6 & 14.4 & 10.5 & 11.5 & 12.4 & 9.06 & 7.25 & 7.53 \\
\hline
\end{tabular}




\begin{tabular}{|c|c|c|c|c|c|c|c|c|c|c|}
\hline $\mathrm{Ta}$ & 0.74 & 0.85 & 0.48 & 0.84 & 0.58 & 0.61 & 0.66 & 0.62 & 0.47 & 0.5 \\
\hline $\mathrm{La}$ & 57.3 & 74.5 & 47.6 & 60.5 & 65.3 & 70.5 & 78.3 & 22.5 & 39.3 & 52.4 \\
\hline $\mathrm{Ce}$ & 113 & 130 & 86.8 & 119 & 113 & 120 & 134 & 40.7 & 66.2 & 94.3 \\
\hline $\operatorname{Pr}$ & 11.1 & 14.9 & 9.76 & 12.9 & 12.4 & 13.4 & 14.9 & 4.82 & 6.79 & 10.2 \\
\hline $\mathrm{Sr}$ & 1089 & 1937 & 1109 & 1616 & 987 & 1047 & 1092 & 1250 & 1443 & 1495 \\
\hline $\mathrm{Nd}$ & 38 & 51.7 & 34.2 & 46.8 & 42.8 & 45.1 & 49 & 18.4 & 22.7 & 35.5 \\
\hline $\mathrm{Zr}$ & 255 & 308 & 201 & 314 & 242 & 258 & 266 & 229 & 198 & 205 \\
\hline $\mathrm{Hf}$ & 6.07 & 6.63 & 5 & 7.02 & 5.82 & 5.91 & 6.19 & 5.44 & 4.66 & 4.99 \\
\hline $\mathrm{Sm}$ & 5.27 & 7.19 & 5.43 & 7.13 & 6.22 & 6.44 & 7.01 & 3.22 & 3.53 & 5.14 \\
\hline $\mathrm{Eu}$ & 1.27 & 1.81 & 1.12 & 2.11 & 1.28 & 1.32 & 1.45 & 1.37 & 1.33 & 1.39 \\
\hline $\mathrm{Gd}$ & 2.83 & 4.11 & 4 & 4.44 & 4.08 & 4.15 & 4.01 & 2.3 & 2.26 & 2.7 \\
\hline $\mathrm{Tb}$ & 0.43 & 0.61 & 0.51 & 0.62 & 0.52 & 0.54 & 0.55 & 0.33 & 0.36 & 0.4 \\
\hline Dy & 2.08 & 2.9 & 2.8 & 3.18 & 2.69 & 2.63 & 2.61 & 1.86 & 1.97 & 2.03 \\
\hline $\mathrm{Y}$ & 9.89 & 14.8 & 14.8 & 14.9 & 14 & 14.3 & 13.9 & 8.7 & 9.77 & 9.78 \\
\hline Ho & 0.37 & 0.52 & 0.5 & 0.58 & 0.48 & 0.46 & 0.46 & 0.35 & 0.38 & 0.38 \\
\hline $\mathrm{Er}$ & 0.95 & 1.33 & 1.3 & 1.48 & 1.21 & 1.2 & 1.19 & 0.93 & 1 & 0.99 \\
\hline $\mathrm{Tm}$ & 0.13 & 0.19 & 0.21 & 0.2 & 0.18 & 0.17 & 0.17 & 0.13 & 0.15 & 0.14 \\
\hline $\mathrm{Yb}$ & 0.77 & 1.25 & 1.19 & 1.25 & 1.08 & 1.05 & 1.01 & 0.84 & 0.93 & 0.85 \\
\hline $\mathrm{Lu}$ & 0.12 & 0.22 & 0.2 & 0.21 & 0.18 & 0.17 & 0.16 & 0.14 & 0.15 & 0.14 \\
\hline$\left({ }^{87} \mathrm{Sr}{ }^{86} \mathrm{Sr}\right)_{\mathrm{i}}$ & 0.7074 & 0.7071 & 0.7071 & 0.7073 & 0.7070 & & 0.7068 & 0.7070 & 0.7057 & 0.7062 \\
\hline$\left({ }^{143} \mathrm{Nd} /{ }^{144} \mathrm{Nd}\right)_{\mathrm{i}}$ & 0.512356 & 0.512391 & 0.512426 & 0.512378 & 0.512413 & & 0.512404 & 0.512478 & 0.512461 & 0.512494 \\
\hline$\varepsilon \mathrm{Nd}(\mathrm{t})$ & -5.5 & -4.82 & -4.13 & -5.07 & -4.4 & & -4.56 & -3.12 & -3.45 & -2.81 \\
\hline
\end{tabular}

Table S7-continued

\begin{tabular}{|c|c|c|c|c|c|c|c|c|c|c|}
\hline Samples & $7509-3$ & $\begin{array}{c}7509- \\
4\end{array}$ & $\begin{array}{c}\mathrm{CH}- \\
1 \mathrm{~A} \\
\end{array}$ & $\mathrm{Z} 02 \mathrm{H} 1$ & Z07H1 & $\mathrm{Z} 07 \mathrm{H} 2$ & Z07H3 & Z07H4 & Z07H5 & Z07H6 \\
\hline Ref. & 4 & 4 & 5 & 3 & 3 & 3 & 3 & 3 & 3 & 3 \\
\hline $\mathrm{SiO}_{2}$ & 67.48 & 67.53 & 71.86 & 61.13 & 58.71 & 58.3 & 62.09 & 58.44 & 62.31 & 63.58 \\
\hline $\mathrm{TiO}_{2}$ & 0.67 & 0.67 & 0.32 & 0.7 & 0.72 & 0.71 & 0.59 & 0.71 & 0.65 & 0.65 \\
\hline $\mathrm{Al}_{2} \mathrm{O}_{3}$ & 14.74 & 14.8 & 15.2 & 15.62 & 14.03 & 13.95 & 15.24 & 13.92 & 15.3 & 15.28 \\
\hline $\mathrm{Fe}_{2} \mathrm{O}_{3}$ & 3.96 & 4.03 & 1.36 & 4.6 & 5.28 & 5.31 & 5.09 & 5.14 & 4 & 4.85 \\
\hline $\mathrm{MnO}$ & 0.05 & 0.05 & 0.01 & 0.06 & 0.08 & 0.08 & 0.08 & 0.08 & 0.06 & 0.05 \\
\hline $\mathrm{MgO}$ & 2.06 & 2.13 & 0.19 & 3.75 & 5.42 & 5.86 & 4.18 & 5.47 & 4.23 & 3.61 \\
\hline $\mathrm{CaO}$ & 3.43 & 3.49 & 0.23 & 5.6 & 5.99 & 5.9 & 4.89 & 6.31 & 4.62 & 4.58 \\
\hline $\mathrm{Na}_{2} \mathrm{O}$ & 3.46 & 3.47 & 0.69 & 3.79 & 2.89 & 3.09 & 3.47 & 2.96 & 3.12 & 3.41 \\
\hline $\mathrm{K}_{2} \mathrm{O}$ & 4.07 & 4.04 & 7.09 & 3.27 & 3.48 & 3.5 & 3.29 & 3.4 & 3.33 & 3.19 \\
\hline $\mathrm{P}_{2} \mathrm{O}_{5}$ & 0.2 & 0.2 & 0.1 & 0.38 & 0.35 & 0.36 & 0.21 & 0.36 & 0.26 & 0.25 \\
\hline Mg\# & 51 & 51 & 22 & 62 & 67 & 69 & 62 & 68 & 68 & 60 \\
\hline $\mathrm{Cr}$ & 61.9 & 62.5 & 9 & 200 & 285 & 283 & 241 & 289 & 203 & 209 \\
\hline $\mathrm{Ni}$ & 22.5 & 23.6 & 4.4 & 115 & 192 & 200 & 172 & 185 & 108 & 135 \\
\hline $\mathrm{Rb}$ & 159 & 156 & 324 & 100 & 126 & 125 & 108 & 122 & 140 & 116 \\
\hline $\mathrm{Ba}$ & 1009 & 1002 & 2570 & 1531 & 1690 & 1629 & 1877 & 1757 & 1589 & 1505 \\
\hline $\mathrm{Th}$ & 29.1 & 28.3 & 28.4 & 22.6 & 22.7 & 22.7 & 21.4 & 22.5 & 22.9 & 22.3 \\
\hline $\mathrm{Nb}$ & 9.5 & 9.49 & 14.2 & 10.6 & 9.23 & 9.21 & 7.01 & 9.29 & 8.35 & 8.07 \\
\hline $\mathrm{Ta}$ & 0.56 & 0.53 & 0.58 & 0.68 & 0.63 & 0.62 & 0.47 & 0.64 & 0.58 & 0.6 \\
\hline $\mathrm{La}$ & 67.6 & 67.9 & 62.8 & 75.1 & 68.8 & 68.7 & 50.4 & 69.4 & 59.2 & 58 \\
\hline $\mathrm{Ce}$ & 113 & 115 & 97.1 & 137 & 127 & 127 & 90.8 & 128 & 107 & 104 \\
\hline $\mathrm{Pr}$ & 11.9 & 12 & 9.12 & 14.3 & 13.6 & 13.6 & 9.55 & 13.6 & 11.1 & 11 \\
\hline $\mathrm{Sr}$ & 524 & 511 & 690 & 1397 & 1238 & 1230 & 1068 & 1257 & 998 & 978 \\
\hline $\mathrm{Nd}$ & 38.5 & 38.5 & 36.4 & 52.9 & 51.6 & 51.6 & 35.1 & 51.4 & 41.3 & 40.8 \\
\hline $\mathrm{Zr}$ & 275 & 280 & 182 & 233 & 204 & 205 & 189 & 206 & 200 & 196 \\
\hline $\mathrm{Hf}$ & 6.3 & 6.41 & 5.3 & 5.71 & 5.14 & 5.14 & 4.71 & 5.07 & 4.9 & 4.82 \\
\hline $\mathrm{Sm}$ & 5.43 & 5.39 & 5.33 & 7.57 & 7.94 & 7.83 & 5.51 & 7.9 & 6.27 & 6.29 \\
\hline $\mathrm{Eu}$ & 1.02 & 1.03 & 1.08 & 1.84 & 1.92 & 1.9 & 1.42 & 1.9 & 1.55 & 1.54 \\
\hline $\mathrm{Gd}$ & 3.02 & 3.08 & 3.08 & 5.76 & 6.15 & 6.09 & 4.5 & 6.15 & 4.94 & 4.98 \\
\hline $\mathrm{Tb}$ & 0.43 & 0.44 & 0.51 & 0.63 & 0.71 & 0.7 & 0.55 & 0.7 & 0.6 & 0.6 \\
\hline Dy & 2.1 & 2.03 & 2.31 & 3.04 & 3.42 & 3.35 & 2.83 & 3.38 & 3.01 & 3.02 \\
\hline $\mathrm{Y}$ & 10.9 & 10.6 & 11.1 & 15.2 & 17.4 & 17.1 & 16.1 & 17.7 & 16.5 & 15.9 \\
\hline Ho & 0.35 & 0.34 & 0.47 & 0.53 & 0.62 & 0.61 & 0.56 & 0.61 & 0.56 & 0.55 \\
\hline $\mathrm{Er}$ & 0.92 & 0.89 & 1.34 & 1.39 & 1.63 & 1.57 & 1.51 & 1.62 & 1.53 & 1.47 \\
\hline $\mathrm{Tm}$ & 0.12 & 0.13 & 0.21 & 0.2 & 0.23 & 0.23 & 0.23 & 0.23 & 0.22 & 0.21 \\
\hline $\mathrm{Yb}$ & 0.76 & 0.79 & 1.41 & 1.22 & 1.37 & 1.35 & 1.43 & 1.39 & 1.38 & 1.29 \\
\hline $\mathrm{Lu}$ & 0.12 & 0.12 & 0.19 & 0.18 & 0.21 & 0.2 & 0.21 & 0.21 & 0.2 & 0.19 \\
\hline
\end{tabular}




\begin{tabular}{|c|c|l|l|l|l|c|c|c|c|c|}
\hline$\left({ }^{87} \mathrm{Sr}^{86} \mathrm{Sr}\right)_{\mathrm{i}}$ & 0.7082 & & & & & 0.7075 & 0.7075 & & & 0.7073 \\
\hline$\left({ }^{143} \mathrm{Nd} /{ }^{144} \mathrm{Nd}\right)_{\mathrm{i}}$ & 0.512356 & & & & & 0.512347 & 0.512375 & & & 0.512337 \\
\hline$\varepsilon \mathrm{Nd}(\mathrm{t})$ & -5.51 & & & & & -5.68 & -5.13 & & -5.87 \\
\hline
\end{tabular}

Table S7-continud

\begin{tabular}{|c|c|c|c|c|c|c|}
\hline Samples & Z08H5 & $\mathrm{Z12H1}$ & $\mathrm{Z} 12 \mathrm{H} 2$ & $\mathrm{CH}-2 \mathrm{~A}$ & CH-3A & $\mathrm{CH}-4 \mathrm{~A}$ \\
\hline Reference & 3 & 3 & 3 & 5 & 5 & 5 \\
\hline $\mathrm{SiO}_{2}$ & 64.69 & 60.24 & 62.94 & 70.52 & 69.81 & 70.48 \\
\hline $\mathrm{TiO}_{2}$ & 0.72 & 0.73 & 0.78 & 0.31 & 0.36 & 0.34 \\
\hline $\mathrm{Al}_{2} \mathrm{O}_{3}$ & 16.23 & 15.82 & 17.09 & 14.48 & 14.89 & 14.62 \\
\hline $\mathrm{Fe}_{2} \mathrm{O}_{3}$ & 3.21 & 6.01 & 3.07 & 2.81 & 3.3 & 3.25 \\
\hline $\mathrm{MnO}$ & 0.02 & 0.06 & 0.06 & 0.05 & 0.08 & 0.06 \\
\hline $\mathrm{MgO}$ & 1.05 & 2 & 1.06 & 0.5 & 0.62 & 0.56 \\
\hline $\mathrm{CaO}$ & 4.09 & 6.02 & 5.28 & 1.98 & 1.84 & 1.89 \\
\hline $\mathrm{Na}_{2} \mathrm{O}$ & 3.86 & 3.66 & 4.12 & 3.91 & 3.48 & 0.35 \\
\hline $\mathrm{K}_{2} \mathrm{O}$ & 3.55 & 2.98 & 3.24 & 4.2 & 4.32 & 4.59 \\
\hline $\mathrm{P}_{2} \mathrm{O}_{5}$ & 0.33 & 0.27 & 0.29 & 0.17 & 0.19 & 0.16 \\
\hline $\mathrm{Mg} \#$ & 40 & 40 & 41 & 26 & 27 & 26 \\
\hline $\mathrm{Cr}$ & 278 & 241 & 258 & 10.2 & 12 & 30.5 \\
\hline $\mathrm{Ni}$ & 81 & 125 & 78.5 & 9.4 & 8.25 & 8.4 \\
\hline $\mathrm{Rb}$ & 118 & 110 & 117 & 166 & 171 & 173 \\
\hline $\mathrm{Ba}$ & 1665 & 1280 & 1358 & 1290 & 1475 & 1450 \\
\hline Th & 25.9 & 17 & 17.7 & 27.9 & 26 & 24.3 \\
\hline $\mathrm{Nb}$ & 9.25 & 8.31 & 8.81 & 15.4 & 16 & 14.2 \\
\hline $\mathrm{Ta}$ & 0.63 & 0.59 & 0.63 & 1.75 & 1.84 & 0.94 \\
\hline $\mathrm{La}$ & 76.5 & 46.2 & 48.5 & 80.4 & 95.9 & 76.4 \\
\hline $\mathrm{Ce}$ & 132 & 85 & 87.9 & 132 & 133 & 117 \\
\hline $\mathrm{Pr}$ & 14.3 & 9.26 & 9.51 & 11.6 & 13 & 11.1 \\
\hline $\mathrm{Sr}$ & 1228 & 927 & 972 & 1020 & 885 & 1010 \\
\hline $\mathrm{Nd}$ & 54 & 35.4 & 36.1 & 44.1 & 49.3 & 41.3 \\
\hline $\mathrm{Zr}$ & 217 & 179 & 184 & 197 & 195 & 181 \\
\hline $\mathrm{Hf}$ & 5.5 & 4.51 & 4.73 & 5.38 & 5.46 & 4.71 \\
\hline $\mathrm{Sm}$ & 8.09 & 5.84 & 5.76 & 6.3 & 7.11 & 5.97 \\
\hline $\mathrm{Eu}$ & 1.97 & 1.55 & 1.62 & 1.27 & 1.46 & 1.28 \\
\hline $\mathrm{Gd}$ & 6.32 & 4.83 & 4.63 & 3.85 & 4.7 & 3.93 \\
\hline $\mathrm{Tb}$ & 0.73 & 0.61 & 0.57 & 0.57 & 0.81 & 0.62 \\
\hline Dy & 3.5 & 3.25 & 2.82 & 2.74 & 3.36 & 2.97 \\
\hline $\mathrm{Y}$ & 16.8 & 17.1 & 14.6 & 11.7 & 14.3 & 12.4 \\
\hline Ho & 0.61 & 0.61 & 0.52 & 0.54 & 0.65 & 0.64 \\
\hline $\mathrm{Er}$ & 1.54 & 1.67 & 1.32 & 4.41 & 1.86 & 1.84 \\
\hline $\mathrm{Tm}$ & 0.22 & 0.24 & 0.2 & 0.23 & 0.28 & 0.28 \\
\hline $\mathrm{Yb}$ & 1.28 & 1.5 & 1.1 & 1.48 & 1.74 & 1.51 \\
\hline $\mathrm{Lu}$ & 0.18 & 0.22 & 0.16 & 0.19 & 0.22 & 0.21 \\
\hline$\left({ }^{87} \mathrm{Sr} /{ }^{86} \mathrm{Sr}\right)_{\mathrm{i}}$ & 0.7075 & & 0.7074 & & & \\
\hline$\left({ }^{143} \mathrm{Nd}^{1 / 44} \mathrm{Nd}\right)_{4}$ & 0.51235 & & 0.512433 & & & \\
\hline$\varepsilon N d(t)$ & -5.62 & & -4 & & & \\
\hline
\end{tabular}

1. Ou, Q., Wang, Q., Wyman, D.A., Zhang, H.X., Yang, J.H., Zeng, J.P., Hao, L.L., Chen, Y.W., Liang, H., and Qi, Y., 2017, Eocene adakitic porphyries in the central- north Qiangtang Terrane, central Tibet: partial melting of thickened lower crust and implications for initial surface uplifting of the plateau. Journal of Geophysical Research, Solid Earth, 122, 10251053.

2. Chen, J., Xu, J., Dong, Y., Wang, B., and Kang, Z., 2013, Geochemistry of Eocene high-Mg\# adakitic rocks in the northern Qiangtang terrane, central Tibet: implications for early delamination of lower crustal and early uplifting of in center the plateau: Geological Society of America Bulletin, v. 125, p. 1800-1819. 
3. Lai, S., and Qin, J., 2013, Adakitic rocks derived from the partial melting of subducted continental crust: Evidence from the Eocene volcanic rocks in the northern Qiangtang block: Gondwana Research, v. 23, p. 812-824.

4. Wang, Q., Wyman, D.A., Xu, J., Dong, Y., Vasconcelos, P.M., Pearson, N., Wan, Y., Li, C., Yu, Y., Zhu, T., Feng, X., Zhang, Q., Zi, F., and Chu, Z., 2008, Eocene melting of subducting continental crust and early uplifting of central Tibet:Evidence from central-western Qiangtang high-K calc-alkaline andesites, dacites and rhyolites: Earth and Planetary Science Letters, v. 272, p. 158-171.

5. Li, L., Bai,Y., Niu, Z., Yao, and H., 2004, Characteristics of the Laorite Co granite porphyry in central Qiangtang, northern Tibet and its tectonic significance: Geological Bulletin of China, v.23, p.1040-1045 (in Chinese with English abstract). 
Table S8- Metadata of LA-MC-ICPMS for U-Pb and Lu-Hf isotope analyses used in this study

\begin{tabular}{|c|c|}
\hline \multicolumn{2}{|l|}{ Sample Preparation } \\
\hline Sample type/mineral & Magmatic zircons \\
\hline Sample preparation & $\begin{array}{l}\text { Conventional mineral separation, } 1 \text { inch resin mount, } 1 \mathrm{~mm} \\
\text { polish to finish }\end{array}$ \\
\hline Imaging & $\begin{array}{l}\text { CL, Gatan CL4+ - FEI Quanta450, } 10 \mathrm{nA}, 17 \mathrm{~mm} \text { working } \\
\text { distance }\end{array}$ \\
\hline \multicolumn{2}{|l|}{ LA-ICPMS U-Pb analyses } \\
\hline Laboratory name & $\begin{array}{l}\text { Guangxi Key Laboratory of Hidden Metallic Ore Deposits } \\
\text { Exploration, Guilin University of Technology }\end{array}$ \\
\hline \multicolumn{2}{|l|}{ Laser ablation system } \\
\hline Make, Model and type & Coherent, GeoLas 193 ArF excimer \\
\hline Ablation cell and volume & In-house built low-volume cell, volume $c a .14 \mathrm{~cm}^{3}$ \\
\hline Laser wavelength (nm) & $193 \mathrm{~nm}$ \\
\hline Pulse width (ns) & $15 \mathrm{~ns}$ \\
\hline Fluence $\left(\mathrm{J} \mathrm{cm}^{-2}\right)$ & $10 \mathrm{~J} \mathrm{~cm}^{-2}$ \\
\hline Repetition rate $(\mathrm{Hz})$ & $6 \mathrm{~Hz}$ \\
\hline Ablation duration (s) & $50 \mathrm{~S}$ \\
\hline Ablation pit depth / ablation rate & not available \\
\hline Spot diameter (mm) nominal/actual & $32 \mathrm{~mm}$ \\
\hline Sampling mode / pattern & Static spot ablation \\
\hline Carrier gas & $\begin{array}{l}100 \% \mathrm{He} \text { in the cell, Argon was introduced into the make-up gas } \\
\text { via a T-connector }\end{array}$ \\
\hline Cell carrier gas flow $\left(1 \mathrm{~min}^{-1}\right)$ & ca. $0.70 \mathrm{~L} / \mathrm{min}$; fine-adjusted daily \\
\hline \multicolumn{2}{|l|}{ ICP-MS Instrument } \\
\hline Make, Model and type & Agilent, 7500a quadrupole ICP-MS \\
\hline Sample introduction & dry aerosol by laser ablation \\
\hline RF power (W) & $1350 \mathrm{~W}$ \\
\hline Make-up gas flow $\left(1 \mathrm{~min}^{-1}\right)$ & ca. $0.65 \mathrm{~L} / \mathrm{min}$; fine-adjusted daily \\
\hline Detection system & dual (pulse and analog) electron multipliers \\
\hline
\end{tabular}




\begin{tabular}{|c|c|}
\hline Masses measured & ${ }^{29} \mathrm{Si},{ }^{91} \mathrm{Zr},{ }^{202} \mathrm{Hg},{ }^{204}(\mathrm{Hg}+\mathrm{Pb}),{ }^{206} \mathrm{~Pb},{ }^{207} \mathrm{~Pb},{ }^{208} \mathrm{~Pb},{ }^{232} \mathrm{Th},{ }^{238} \mathrm{U}$ \\
\hline $\begin{array}{l}\text { Integration time per peak/dwell times } \\
\text { (ms); quadrupole settling time between } \\
\text { mass jumps }\end{array}$ & $\begin{array}{l}5 \mathrm{~ms} \text { for } 29,91 \text { and } 202 ; 10 \mathrm{~ms} \text { for } 232 \text { and } 238 ; ; 20 \mathrm{~ms} \text { for } 204, \\
206,207 \text { and } 208 ; \text { settling time are allocated automatically by } \\
\text { Agilent ICPMS software }\end{array}$ \\
\hline $\begin{array}{l}\text { Total integration time per output data point } \\
\text { (s) }\end{array}$ & $0.2772 \mathrm{~s}$ \\
\hline 'Sensitivity' as useful yield (\%, element) & not available for quadrupole ICPMS \\
\hline IC Dead time (ns) & 30ns for EM of quadrupole ICPMS \\
\hline \multicolumn{2}{|l|}{ Data Processing } \\
\hline Gas blank & $20 \mathrm{~s}$ on-peak zero subtracted \\
\hline Calibration strategy & $\begin{array}{l}\text { Plesovice zircon used as calibration standard, GJ-1 zircon used } \\
\text { as quality control for validation }\end{array}$ \\
\hline \multirow[t]{2}{*}{ Reference Material info } & Plesovice zircon (Slám et al., 2008) \\
\hline & GJ-1 zircon (Jackson et al. 2004) \\
\hline $\begin{array}{l}\text { Data processing package used / Correction } \\
\text { for LIEF }\end{array}$ & $\begin{array}{l}\text { Commercial software ICPMSDataCal was intially developed by } \\
\text { Prof. Yong-sheng LIU of GUG. LIEF correction assumes } \\
\text { reference material and samples behave identically, namely } \\
\text { standard-samples-standard bracketing (Liu et al., 2008; Liu et al., } \\
\text { 2010). }\end{array}$ \\
\hline Mass discrimination & not available \\
\hline $\begin{array}{l}\text { Common- } \mathrm{Pb} \text { correction, composition and } \\
\text { uncertainty }\end{array}$ & Using the method proposed by Andersen (2002). \\
\hline Uncertainty level and propagation & $\begin{array}{l}\text { Ages are quoted at 2s absolute, propagation is processed by the } \\
\text { ICPMSDataCal software. Reproducibility and age uncertainty of } \\
\text { reference material are propagated. }\end{array}$ \\
\hline Quality control / Validation & $\begin{array}{l}\text { GJ-1 - Weighted ave }{ }^{206} \mathrm{~Pb} /{ }^{238} \mathrm{U} \text { age }=605 \pm 3(2 \sigma, \mathrm{MSWD}= \\
0.4, \mathrm{n}=24) \\
\text { Systematic uncertainty for propagation is } 1.5 \%(1 s) .\end{array}$ \\
\hline Other information & $\begin{array}{l}\text { ca. } 2 \mathrm{~m} \text { sample line from ablation cell to torch and washout time } \\
\text { was } c a .10 \mathrm{~s} \text { to } 0.1 \% \text { of peak signal. }\end{array}$ \\
\hline
\end{tabular}




\begin{tabular}{|c|c|}
\hline LA-MC-ICPMS Lu-Hf analyses & \\
\hline \multicolumn{2}{|l|}{ Laboratory and Sample Preparation } \\
\hline Laboratory name & $\begin{array}{l}\text { Guangxi Key Laboratory of Hidden Metallic Ore Deposits } \\
\text { Exploration, Guilin University of Technology }\end{array}$ \\
\hline \multicolumn{2}{|l|}{ Laser ablation system } \\
\hline Make, Model and type & Coherent, GeoLas $193 \mathrm{ArF}$ excimer \\
\hline Ablation cell and volume & In-house built low-volume cell, volume ca. $14 \mathrm{~cm} 3$ \\
\hline Laser wavelength (nm) & $193 \mathrm{~nm}$ \\
\hline Pulse width (ns) & $15 \mathrm{~ns}$ \\
\hline Fluence $\left(\mathrm{J} \mathrm{cm}^{-2}\right)$ & $10 \mathrm{~J} \mathrm{~cm}^{-2}$ \\
\hline Repetition rate $(\mathrm{Hz})$ & $6 \mathrm{~Hz}$ \\
\hline Ablation duration (s) & $47 \mathrm{~s}$ \\
\hline Ablation pit depth / ablation rate & about $15 \mu \mathrm{m}$ \\
\hline Spot diameter $(\mathrm{mm})$ nominal/actual & $44 \mu \mathrm{m}$ \\
\hline Sampling mode / pattern & Static spot ablation \\
\hline Carrier gas & $\begin{array}{l}100 \% \mathrm{He} \text { in the cell, } \mathrm{N}_{2} \text { were introduced into the make-up gas } \\
\text { via a T-connector }\end{array}$ \\
\hline Cell carrier gas flow $\left(1 \mathrm{~min}^{-1}\right)$ & $0.64 \mathrm{~L} / \mathrm{min}$ \\
\hline \multicolumn{2}{|l|}{ MC-ICP-MS Instrument } \\
\hline Make, Model and type & Thermo, Neptune Plus MC-ICP-MS \\
\hline Sample introduction & dry aerosol by laser ablation \\
\hline Make-up gas flow $\left(1 \mathrm{~min}^{-1}\right)$ & ca. $0.65 / \mathrm{min}$; fine-adjusted daily \\
\hline Detection system & Faraday cups \\
\hline Masses measured & ${ }^{172} \mathrm{Yb},{ }^{173} \mathrm{Yb},{ }^{175} \mathrm{Lu},{ }^{176}(\mathrm{Hf}+\mathrm{Yb}+\mathrm{Lu}),{ }^{177} \mathrm{Hf},{ }^{178} \mathrm{Hf},{ }^{179} \mathrm{Hf},{ }^{180} \mathrm{Hf}$ \\
\hline $\begin{array}{l}\text { Integration time per peak/dwell times } \\
\text { (ms); quadrupole settling time between } \\
\text { mass jumps }\end{array}$ & $0.131 \mathrm{~s}$ \\
\hline $\begin{array}{l}\text { Total integration time per output data point } \\
\text { (s) }\end{array}$ & $26-30 \mathrm{~s}$ \\
\hline 'Sensitivity’ as useful yield (\%, element) & not available \\
\hline IC Dead time (ns) & not available \\
\hline
\end{tabular}




\begin{tabular}{|c|c|}
\hline Data Processing & \\
\hline Gas blank & 20 s on-peak zero subtracted \\
\hline Calibration strategy & $\begin{array}{l}\text { GJ-1 zircon used as external standard, PengLai zircon used as } \\
\text { unknown sampels }\end{array}$ \\
\hline Reference Material info & $\begin{array}{l}\text { PengLai (Li et al., 2010) } \\
\text { GJ-1 (Elhlou et al., 2006) }\end{array}$ \\
\hline $\begin{array}{l}\text { Data processing package used / Correction } \\
\text { for LIEF }\end{array}$ & $\begin{array}{l}\text { Commercial software ICPMSDataCal was intially developed by } \\
\text { Prof. Yong-sheng LIU of GUG. LIEF correction assumes } \\
\text { reference material and samples behave identically, namely } \\
\text { standard-samples-standard bracketing (Liu et al., 2008; Liu et al., } \\
2010 \text { ). }\end{array}$ \\
\hline Mass discrimination & $\begin{array}{l}{ }^{175} \mathrm{Lu} /{ }^{176} \mathrm{Lu}=0.02655 \\
{ }^{172} \mathrm{Yb} /{ }^{176} \mathrm{Yb}=0.5886, \beta_{\mathrm{Yb}}=0.8725 \times \beta_{\mathrm{Hf}}\end{array}$ \\
\hline Uncertainty level and propagation & $\begin{array}{l}\text { Isotope values are quoted at } 2 \mathrm{~s} \text { absolute, propagation is } \\
\text { processed by the ICPMSDataCal software. Reproducibility and } \\
\text { age uncertainty of reference material are propagated. }\end{array}$ \\
\hline Quality control / Validation & $\begin{array}{l}\text { GJ-1 }- \text { Weighted ave }{ }^{177} \mathrm{Hf} /{ }^{176} \mathrm{Hf}=0.282005 \pm 0.000005(2 \mathrm{~s} \\
\mathrm{MSWD}=0.75 ; \mathrm{n}=32) ; \text { PengLai }- \text { weighted ave } 177 \mathrm{Hf} / 176 \mathrm{Hf}= \\
0.282897 \pm 9(2 \mathrm{~s}, \mathrm{n}=16, \mathrm{MSWD}=0.52) . \\
\text { Systematic uncertainty for propagation is } 0.0015 \%(1 \mathrm{~s}) .\end{array}$ \\
\hline Other information & $\begin{array}{l}\text { ca. } 2 \mathrm{~m} \text { sample line from ablation cell to torch and washout time } \\
\text { was } c a .1 \mathrm{~s} \text { to } 1 \% \text { of peak signal. }\end{array}$ \\
\hline
\end{tabular}

